\title{
The global existence and large time behavior of smooth compressible fluid in an infinitely expanding ball, I: 3D Euler equations
}

\author{
Gang $\mathrm{Xu}^{1}$, Huicheng $\mathrm{Yin}^{2 *}$ \\ 1. Faculty of Science, Jiangsu University, Zhenjiang, Jiangsu 212013, China. \\ 2. School of Mathematical Sciences, Jiangsu Provincial Key Laboratory for Numerical Simulation \\ of Large Scale Complex Systems, Nanjing Normal University, Nanjing 210023, China.
}

\begin{abstract}
We concern with the global existence and large time behavior of compressible fluids (including the inviscid gases, viscid gases, and Boltzmann gases) in an infinitely expanding ball. Such a problem is one of the interesting models in studying the theory of global smooth solutions to multidimensional compressible gases with time dependent boundaries and vacuum states at infinite time. Due to the conservation of mass, the fluid in the expanding ball becomes rarefied and eventually tends to a vacuum state meanwhile there are no appearances of vacuum domains in any part of the expansive ball, which is easily observed in finite time. In this paper, as the first part of our three papers, we will confirm this physical phenomenon for the compressible inviscid fluids by obtaining the exact lower and upper bound on the density function.
\end{abstract}

Keywords: Compressible Euler equations, expanding ball, global existence, degenerate, weighted energy estimates, large time behavior

Mathematical Subject Classification 2000: 35L70, 35L65, 35L67, 76N15

\section{Introduction}

In this paper, we consider the behavior of a compressible inviscid fluid in a 3D expanding ball given by $\Omega_{0}=\left\{(t, x): t \geq 0,|x|=\sqrt{x_{1}^{2}+x_{2}^{2}+x_{3}^{2}} \leq R_{0}(t)\right\}$, where $R_{0}(t) \in C^{6}[0, \infty)$ satisfies $R_{0}(0)=$ $1, R_{0}^{\prime}(0)=0, R_{0}^{\prime \prime}(0)=0$, and $R_{0}(t)=1+L t$ for $t \geq 1$ with some positive constant $L$. From the expression of $\Omega_{0}$, we know that the ball $S_{t}^{0}=\left\{x:|x| \leq R_{0}(t)\right\}$ at the time $t$ is artificially set by pulling out the initial unit ball $S^{0}=\{x:|x| \leq 1\}$ with a smooth speed and acceleration (see Figure 1 below).

Suppose that the movement of fluid in the ball is governed by the 3D compressible isentropic Euler

\footnotetext{
${ }^{*}$ Gang Xu (gxu@ujs.edu.cn) and Huicheng Yin (huicheng@nju.edu.cn) are supported by the National Natural Science Foundation of China (No.11025105, No.11571177) and a project funded by the Priority Academic Program Development of Jiangsu Higher Education Institutions.
} 


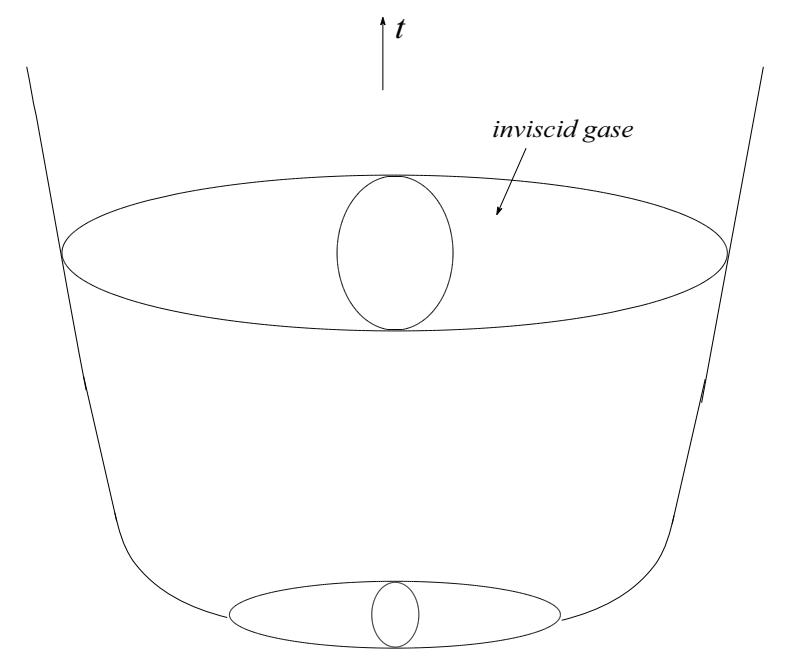

Figure 1: An inviscid flow in a 3D expanding ball

system:

$$
\left\{\begin{array}{l}
\partial_{t} \rho+\sum_{i=1}^{3} \partial_{i}\left(\rho u_{i}\right)=0 \\
\partial_{t}\left(\rho u_{i}\right)+\sum_{j=1}^{3} \partial_{j}\left(\rho u_{i} u_{j}\right)+\partial_{i} P=0, \quad i=1,2,3
\end{array}\right.
$$

where $x=\left(x_{1}, x_{2}, x_{3}\right), \rho, u=\left(u_{1}, u_{2}, u_{3}\right)$ and $P$ represent the density, velocity and pressure, respectively. Moreover, assume the state equation $P=A \rho^{\gamma}$ holds with $A>0$ and $\gamma\left(1<\gamma<\frac{4}{3}\right)$ being constants. Without loss of generality, take $A=1$ and impose the following initial-boundary conditions on $(1.1)$

$$
\begin{cases}\rho(0, x)=\rho_{0}(x), u(0, x)=u_{0}(x), & \text { for } x \in S^{0}, \\ R^{\prime}(t)=\sum_{i=1}^{3} \frac{x_{i} u_{i}}{|x|}, & \text { for }(t, x) \in \partial \Omega_{0}=\left\{(t, x): t \geq 0,|x|=R_{0}(t)\right\},\end{cases}
$$

where $\rho_{0}(x) \in H^{4}\left(S^{0}\right), u_{0}(x) \in H_{0}^{4}\left(S^{0}\right)$, and $\rho_{0}(x)>0$ for $x \in S^{0}$. Here, the boundary condition on $\partial \Omega_{0}$ in (1.2) represents the solid wall.

The purpose of this paper is to prove the following theorem.

Theorem 1.1 If $\rho_{0}(x) \in H^{4}\left(S^{0}\right), u_{0}(x) \in H_{0}^{4}\left(S^{0}\right)$, rot $u_{0}(x) \equiv 0$, and the compatibility conditions on $\left\{(t, x): t=0, x \in \partial S^{0}\right\}$ of $\left(\rho_{0}(x), u_{0}(x)\right)$ hold, then there exist a constant $h_{0}>0$, and a small constant $\varepsilon_{0}>0$ depending only on $h_{0}$, such that when $\sup _{0 \leq t \leq 1,1 \leq k \leq 5}\left|R_{0}^{(k)}(t)\right|+\left\|\rho_{0}(x)-1\right\|_{H^{4}\left(S^{0}\right)}+$ $\left\|u_{0}(x)\right\|_{H^{4}\left(S^{0}\right)}<\varepsilon_{0}, R_{0}(t)=1+$ Lt for $t \geq 1$, and $0<L<h_{0}$, problem (1.1)-(1.2) with $1<\gamma<\frac{4}{3}$ admits a global solution $(\rho, u)$ in $\Omega_{0}$ satisfying

$$
\begin{aligned}
& (\rho(t, x), u(t, x)) \in C\left([0, \infty), H^{4}\left(S_{t}^{0}\right)\right) \cap C^{1}\left([0, \infty), H^{3}\left(S_{t}^{0}\right)\right), \\
& \operatorname{rot} u(t, x) \equiv 0, \quad(t, x) \in \Omega_{0}, \\
& \frac{1}{2 R^{3}(t)} \leq \rho(t, x) \leq \frac{3}{2 R^{3}(t)} \quad \text { for } \quad t \geq 1,
\end{aligned}
$$


and

$$
\sup _{x \in S_{t}^{0}}\left(\left|u(t, x)-\frac{L x}{R(t)}\right|+\left|R(t) \nabla\left(u(t, x)-\frac{L x}{R(t)}\right)\right|\right) \rightarrow 0 \text { as } t \rightarrow+\infty .
$$

Here, $S_{t}^{0}=\left\{x:|x| \leq R_{0}(t)\right\}$, and $R(t)=1+$ Lt for $t \geq 0$.

In the following, we give some remarks on the above theorem. of mass.

Remark 1.1. The pointwise estimate $\rho(t, x) \sim \frac{1}{R^{3}(t)}$ for large $t$ can be expected by the conservation

Remark 1.2. When the fluid is governed by the compressible Navier-Stokes equations and the corresponding boundary condition is given by $u(t, x)=\frac{R^{\prime}(t) x}{R(t)}$ for $(t, x) \in \partial \Omega_{0}$, the corresponding result was obtained in [25]. The case for a rarefied gas in $\Omega_{0}$ governed by the Boltzmann equation was also obtained in [26].

Remark 1.3. If the initial density contains vacuum, the local well-posedness of the compressible Euler system have been extensively studied, cf. [3], [7-8], [11-12], [16], [18] and the references therein. In general, classical solutions will blow up in finite time as shown in [3] and [23]. For the problem considered in this paper, the vacuum is the time asymptotic state so that the classical solution exists globally in time.

Remark 1.4. When the initial velocity $u_{0}(x)$ is close to a linear field, the authors in [10] and [19] proved the global existence of smooth solution to the Cauchy problem of the compressible Euler system. And this is different from the case considered in this paper.

Remark 1.5. If the ball $S^{0}$ is pulled outwards rapidly, namely, when the number $L$ is large, part of the region inside the ball may become vacuum in finite time (see [6] and so on).

Remark 1.6. Note that $(\hat{\rho}(t, x), \hat{u}(t, x))=\left(\frac{1}{R^{3}(t)}, \frac{L x}{R(t)}\right)$ with $R(t)=1+$ Lt is a special solution to (1.1)-(1.2). In fact, Theorem 1.2 gives the stability of this special solution. In addition, the smallness of $L$ in Theorem 1.1 is only used to prove the local existence of the solution to (1.1)-(1.2) and to obtain the smallness of $(\rho(1, x)-1, u(1, x))$ in (6.11).

Remark 1.7. For the spherically symmetric solution, one can relax the restriction on $1<\gamma<\frac{4}{3}$ in Theorem 1.1 to $1<\gamma<\frac{5}{3}$. The main reasons come from the simplified boundary conditions (5.66) and (5.112) in Section 5, and the assumption (5.2) in the domain $\left\{(t, x): t>0, r>\frac{1}{3} R(t)\right\}$ can be replaced by

$$
\left\{\begin{array}{l}
|Z \dot{\Phi}| \leq M \varepsilon R(t)^{-\sigma} \quad \text { if } \gamma \in\left(1, \frac{4}{3}\right) \cup\left(\frac{4}{3}, \frac{5}{3}\right) ; \quad|Z \dot{\Phi}| \leq M \varepsilon \ln R(t) \quad \text { if } \gamma=\frac{4}{3} \\
\left|Z D_{t} \dot{\Phi}\right| \leq M \varepsilon R(t)^{-3(\gamma-1)}
\end{array}\right.
$$

where $\sigma=\min \{0,3(\gamma-1)-1\}$. We omit the details of the estimation for this special case.

To prove Theorem 1.1, we first solve an unsteady potential flow equation in the domain $\Omega=\{(t, x)$ : $t \geq 0,|x| \leq R(t)\}$ with the initial-boundary conditions (1.2) (see the Figure 2 below). Let $\Phi(t, x)$ be the potential of velocity $u=\left(u_{1}, u_{2}, u_{3}\right)$, i.e., $u_{i}=\partial_{i} \Phi(1 \leq i \leq 3)$. Then it follows from the Bernoulli law that

$$
\partial_{t} \Phi+\frac{1}{2}\left|\nabla_{x} \Phi\right|^{2}+h(\rho)=B_{0}
$$


where $h(\rho)=\frac{c^{2}(\rho)}{\gamma-1}$ is the specific enthalpy, $c(\rho)=\sqrt{P^{\prime}(\rho)}$ is the local sound speed, $\nabla_{x}=\left(\partial_{1}, \partial_{2}, \partial_{3}\right)$, $B_{0}=\frac{c^{2}\left(\rho_{0}\right)}{\gamma-1}$ is the Bernoulli constant of a static state with the constant density $\rho_{0}$.

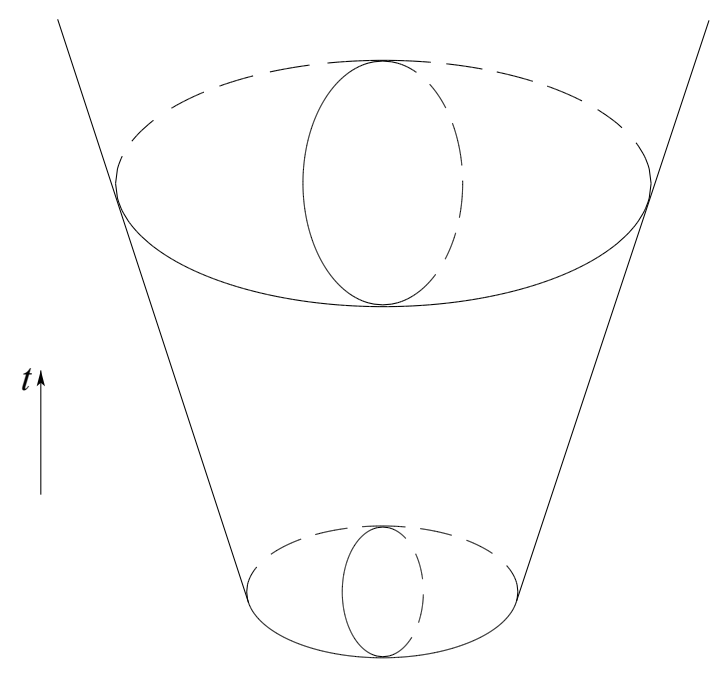

Figure 2:

By (1.7) and the implicit function theorem with $h^{\prime}(\rho)=\frac{c^{2}(\rho)}{\rho}>0$ for $\rho>0$, the density function $\rho(t, x)$ can be expressed as

$$
\rho=h^{-1}\left(B_{0}-\partial_{t} \Phi-\frac{1}{2}\left|\nabla_{x} \Phi\right|^{2}\right) \equiv H(\nabla \Phi),
$$

where $h^{-1}$ stands for the inverse function of $h(\rho)$, and $\nabla=\left(\partial_{t}, \nabla_{x}\right)$.

Substituting (1.8) into the first equation in (1.1) yields

$$
\partial_{t}(H(\nabla \Phi))+\sum_{i=1}^{3} \partial_{i}\left(H(\nabla \Phi) \partial_{i} \Phi\right)=0 .
$$

In fact, for any $C^{2}$ solution $\Phi,(1.9)$ can be rewritten into the following second order quasilinear equation

$$
\partial_{t}^{2} \Phi+2 \sum_{k=1}^{3} \partial_{k} \Phi \partial_{t k}^{2} \Phi+\sum_{i, j=1}^{3} \partial_{i} \Phi \partial_{j} \Phi \partial_{i j}^{2} \Phi-c^{2}(\rho) \Delta \Phi=0 .
$$

Denote the lateral boundary of $\Omega$ by $\partial \Omega=\{(t, x): t \geq 0,|x|=R(t)\}$. Then on $\partial \Omega$,

$$
\sum_{i=1}^{3} \partial_{i} \Phi \cdot \frac{x_{i}}{|x|}=L
$$

Due to the geometric property of $\Omega$, it is convenient to work in the spherical coordinates $(r, \theta, \varphi)$ :

$$
\left(x_{1}, x_{2}, x_{3}\right)=(r \cos \theta \sin \varphi, r \sin \theta \sin \varphi, r \cos \varphi),
$$


where $r=\sqrt{x_{1}^{2}+x_{2}^{2}+x_{3}^{2}}, 0 \leq \theta \leq 2 \pi, 0 \leq \varphi \leq \frac{\pi}{2}$. Under the coordinate transformation (1.12), (1.10) becomes

$$
\begin{aligned}
\partial_{t}^{2} \Phi & +2 \partial_{r} \Phi \partial_{t r}^{2} \Phi+\frac{2}{r^{2}} \partial_{\varphi} \Phi \partial_{t \varphi}^{2} \Phi+\frac{2}{r^{2} \sin ^{2} \varphi} \partial_{\theta} \Phi \partial_{t \theta}^{2} \Phi+\left(\left(\partial_{r} \Phi\right)^{2}-c^{2}(\rho)\right) \partial_{r}^{2} \Phi \\
& +\frac{1}{r^{2} \sin ^{2} \varphi}\left(\frac{\left(\partial_{\theta} \Phi\right)^{2}}{r^{2} \sin ^{2} \varphi}-c^{2}(\rho)\right) \partial_{\theta}^{2} \Phi+\frac{1}{r^{2}}\left(\frac{\left(\partial_{\varphi} \Phi\right)^{2}}{r^{2}}-c^{2}(\rho)\right) \partial_{\varphi}^{2} \Phi+\frac{2 \partial_{r} \Phi \partial_{\theta} \Phi}{r^{2} \sin ^{2} \varphi} \partial_{r \theta}^{2} \Phi \\
& +\frac{2}{r^{2}} \partial_{r} \Phi \partial_{\varphi} \Phi \partial_{r \varphi}^{2} \Phi+\frac{2 \partial_{\theta} \Phi \partial_{\varphi} \Phi}{r^{4} \sin ^{2} \varphi} \partial_{\theta \varphi}^{2} \Phi-\frac{1}{r^{3}}\left(2 r^{2} c^{2}(\rho)+\left(\partial_{\varphi} \Phi\right)^{2}+\frac{\left(\partial_{\theta} \Phi\right)^{2}}{\sin ^{2} \varphi}\right) \partial_{r} \Phi \\
& -\frac{\cot \varphi}{r^{4}}\left(r^{2} c^{2}(\rho)+\frac{\left(\partial_{\theta} \Phi\right)^{2}}{\sin ^{2} \varphi}\right) \partial_{\varphi} \Phi=0 .
\end{aligned}
$$

Note that some coefficients in (1.13) have strong singularities near $\varphi=0$. Consequently, as in [14] and [24], we rewrite (1.13) by introducing some smooth vector fields tangent to the sphere $\mathbb{S}^{2}$.

Set

$$
\left\{\begin{array}{l}
Z_{1}=x_{1} \partial_{2}-x_{2} \partial_{1}=\partial_{\theta} \\
Z_{2}=x_{2} \partial_{3}-x_{3} \partial_{2}=-\cot \varphi \cos \theta \partial_{\theta}-\sin \theta \partial_{\varphi} \\
Z_{3}=x_{3} \partial_{1}-x_{1} \partial_{3}=-\cot \varphi \sin \theta \partial_{\theta}+\cos \theta \partial_{\varphi}
\end{array}\right.
$$

Then it follows from a direct computation that (1.13) can be written as

$$
\begin{aligned}
& \partial_{t}^{2} \Phi+2 \partial_{r} \Phi \partial_{t r}^{2} \Phi+\frac{2}{r^{2}} \sum_{i=1}^{3} Z_{i} \Phi \partial_{t} Z_{i} \Phi+\left(\left(\partial_{r} \Phi\right)^{2}-c^{2}(\rho)\right) \partial_{r}^{2} \Phi \\
& \quad+\frac{2 \partial_{r} \Phi}{r^{2}} \sum_{i=1}^{3} Z_{i} \Phi \partial_{r} Z_{i} \Phi-\frac{c^{2}(\rho)}{r^{2}} \sum_{i=1}^{3} Z_{i}^{2} \Phi+\frac{1}{r^{4}} \sum_{i, j=1}^{3} Z_{i} \Phi Z_{j} \Phi Z_{i} Z_{j} \Phi \\
& \quad+\sum_{i, j=1}^{3} \frac{C_{i j}(\omega)}{r^{3}} \partial_{r} \Phi Z_{i} \Phi Z_{j} \Phi+\sum_{i, j, k=1}^{3} \frac{C_{i j k}(\omega)}{r^{4}} Z_{i} \Phi Z_{j} \Phi Z_{k} \Phi-\frac{2 c^{2}(\rho)}{r} \partial_{r} \Phi=0
\end{aligned}
$$

where $\omega=\frac{x}{r}, C_{i j}(\omega)=C_{i j}\left(\frac{x}{r}\right)$ and $C_{i j k}(\omega)=C_{i j k}\left(\frac{x}{r}\right)$ are smooth functions of their arguments.

Meanwhile, the boundary condition (1.11) becomes

$$
\partial_{r} \Phi=L, \quad \text { on } \partial \Omega
$$

In addition, we impose the following initial perturbation:

$$
\Phi(0, x)=\frac{1}{2} L|x|^{2}+\varepsilon \Phi_{0}(x), \quad \partial_{t} \Phi(0, x)=-\frac{1}{2} L^{2}|x|^{2}+\varepsilon \Phi_{1}(x),
$$

where $\varepsilon>0$ is a small parameter, $\left(\Phi_{0}(x), \Phi_{1}(x)\right) \in\left(H^{5}\left(S^{0}\right), H^{4}\left(S^{0}\right)\right)$, and the initial-boundary value conditions (1.16)-(1.17) are compatible on $S^{0}$. Note that the initial data (1.17) can be replaced by $\left(\Phi(0, x), \partial_{t} \Phi(0, x)\right)=\left(\varepsilon \Phi_{0}(x), \varepsilon \Phi_{1}(x)\right)$ when $L>0$ is small. On the other hand, due to $u_{i}=\partial_{i} \Phi$ and (1.8), the initial conditions (1.17) can be realized by a small perturbation of the initial density and velocity of an irrotational flow. 
Theorem 1.2 Under the above assumptions on the initial and boundary data, if $\gamma \in\left(1, \frac{4}{3}\right)$, then there exists a constant $\varepsilon_{0}>0$ depending on $L, B_{0}$ and $\gamma$ such that problem (1.10) with (1.16)-(1.17) has a global solution $\Phi(t, x) \in C\left([0, \infty), H^{5}\left(S_{t}\right)\right) \cap C^{1}\left([0, \infty), H^{4}\left(S_{t}\right)\right)$ for $\varepsilon<\varepsilon_{0}$, where $S_{t}=\{x: r \leq$ $R(t)\}$. Moreover, $\rho(t, x)>0$ and $\lim _{t \rightarrow \infty} \rho(t, x)=0$ hold.

Remark 1.8. The linearized operator of the quasilinear wave equation (1.10) around the special expanding solution has the approximate form of

$$
\partial_{t}^{2}-\frac{\gamma}{(1+L t)^{3(\gamma-1)}}\left(\partial_{1}^{2}+\partial_{2}^{2}+\partial_{3}^{2}\right)+\frac{3 L(\gamma-1)}{1+L t} \partial_{t}
$$

On the other hand, if one considers the Cauchy problem of (1.1) for initial data as a small perturbation of a uniform constant density $\rho_{0}$ and velocity $\left(0,0, q_{0}\right)$, that is,

$$
\left\{\begin{array}{l}
\partial_{t}^{2} \Phi+2 \sum_{k=1}^{3} \partial_{k} \Phi \partial_{t k}^{2} \Phi+\sum_{i, j=1}^{3} \partial_{i} \Phi \partial_{j} \Phi \partial_{i j}^{2} \Phi-c^{2}(\rho) \Delta \Phi=0 \\
\left.\Phi(t, x)\right|_{t=0}=\varepsilon \Phi_{0}(x),\left.\quad \partial_{t} \Phi(t, x)\right|_{t=0}=q_{0}+\varepsilon \Phi_{1}(x), \quad x \in \mathbb{R}^{3},
\end{array}\right.
$$

where $\Phi_{i}(x) \in C_{0}^{\infty}\left(\mathbb{R}^{3}\right)(i=0,1)$, then (1.18) does not fulfill the "null-condition" introduced in [4] and [13]. Therefore, according to the results obtained in [1-2], [5], [20] and [27], classical solution to (1.18) blows up and then shock forms in finite time. Compared this blowup result with Theorem 1.1-1.2, the global existence of smooth solution to (1.10) together with a fixed wall condition comes from the rarefaction property of fluid.

We now give some remarks on the proof of Theorem 1.2. Since the local solvability of problem (1.10) together with (1.16)-(1.17) is known as long as the vacuum does not appear, cf., for example [17], the proof of Theorem 1.2 is based on the continuation argument.

First of all, note that the linearized operator

$$
\mathcal{L}=\partial_{t}^{2}+\frac{2 L r}{R(t)} \partial_{t r}^{2}+\sum_{i, j=1}^{3} \frac{L^{2} x_{i} x_{j}}{R^{2}(t)} \partial_{i j}^{2}-\frac{\gamma}{R^{3(\gamma-1)}(t)} \Delta+\frac{3 L(\gamma-1)}{R(t)}\left(\partial_{t}+\frac{L r}{R(t)} \partial_{r}\right),
$$

cf. (3.2), is different from the corresponding one in [24] which is

$$
\partial_{t}^{2}-\frac{1}{R^{2(\gamma-1)}(t)}\left(\partial_{1}^{2}+\partial_{2}^{2}\right)+\frac{2 L(\gamma-1)}{R(t)} \partial_{t}
$$

The key ingredients in the analysis for the global existence and pointwise estimate are to obtain some weighted energy estimates by choosing some appropriate multiplier and anisotropic weights. For this, we need to

1. show that the solution does not contain vacuum both on the boundary and inside the region;

2. obtain the different time decay rates of the density and velocity of the solution when it tends to vacuum state at infinite time;

3. fully use the Neumann-type boundary condition (1.16) on $\Phi$ by applying the material derivative $D_{t}=\partial_{t}+\frac{L r}{R(t)} \partial_{r}$ on the solution, and estimate the radial derivatives and angular derivatives of $\Phi$, together with some weighted Sobolev interpolation inequalities given in [15]. 
The rest of the paper is organized as follows. In the next section, we will give some basic properties of the background solution and some preliminary weighted Sobolev interpolation inequalities. In Section 3 , we will reformulate problem (1.10) together with (1.16)-(1.17) by decomposing its solution as a sum of the background solution and a small perturbation so that its linearization can be studied clearly. In Section 4, we will establish a uniform weighted energy estimate for the corresponding linear problem, where an appropriate multiplier is constructed. In Section 5, the uniform higher order weighted estimates of $\dot{\Phi}$ are obtained by the analysis on the radial derivatives and angular derivatives of $\dot{\Phi}$, where the domain decomposition technique is applied. In the last section, we complete the proof of Theorem 1.2 by applying the Sobolev embedding theorem and the continuation argument, and then Theorem 1.1 follows from Theorem 1.2 and the local existence result on the problem (1.1)-(1.2).

\section{Background solution and some preliminaries}

In this section, we analyze the background solution to (1.10) with (1.16)-(1.17) when the initial data (1.17) are

$$
\hat{\Phi}(0, x)=\frac{1}{2} L|x|^{2}, \quad \partial_{t} \hat{\Phi}(0, x)=-\frac{1}{2} L^{2}|x|^{2} .
$$

In this case, the density $\rho(x)$ and velocity $u(t, x)=\nabla_{x} \Phi(t, x)$ in $\Omega$ take the form: $\rho(t, x)=\hat{\rho}(t, r)$, $u(t, x)=\frac{x}{r} \hat{U}(t, r)$. Consequently, problem (1.10) with (1.16) and (2.1) is equivalent to

$$
\left\{\begin{array}{l}
r^{2} \partial_{t} \hat{\rho}+\partial_{r}\left(r^{2} \hat{\rho} \hat{U}\right)=0 \\
\partial_{t}\left(r^{2} \hat{\rho} \hat{U}\right)+\partial_{r}\left(r^{2} \hat{\rho} \hat{U}\right)+r^{2} \partial_{r} P=0 \\
\hat{\rho}(0, r)=1, \quad \hat{U}(0, r)=L r
\end{array}\right.
$$

One can easily check that (2.2) has a solution

$$
\hat{\rho}(t, r)=\frac{1}{R^{3}(t)}, \quad \hat{U}(t, r)=\frac{L r}{R(t)} .
$$

Then for $1<\gamma<\frac{4}{3}$, it follows from $u_{i}=\partial_{i} \Phi$, (1.7) and (2.3) that (1.10) with (1.16) and (2.1) has a solution

$$
\hat{\Phi}(t, r)=\frac{\gamma}{(\gamma-1)(4-3 \gamma) L}+B_{0} t+\frac{L r^{2}}{2(1+L t)}-\frac{\gamma}{(\gamma-1)(4-3 \gamma) L}(1+L t)^{4-3 \gamma},
$$

where $B_{0}=\frac{\gamma}{\gamma-1}$.

Next, we include the weighted Sobolev interpolation inequality from [15] that will be used in Lemma 2.4 .

Lemma 2.1 Suppose $s, \tau, p, \alpha, \beta, q, a$ are real numbers, and $j \geq 0, m>0$ are integers, satisfying

$$
\left\{\begin{array}{l}
p, q \geq 1, \frac{j}{m} \leq a \leq 1, s>0, \\
\frac{1}{s}+\frac{\tau}{n}>0, \frac{1}{p}+\frac{\alpha}{n}>0, \frac{1}{q}+\frac{\beta}{n}>0, \\
m-j-\frac{n}{p} \text { is not a non-negative integer. }
\end{array}\right.
$$


There exists a positive constant $C$ such that the following inequality holds for all $v \in C_{0}^{\infty}\left(\mathbb{R}^{n}\right)$ :

$$
\left.\left.|| x\right|^{\tau} \nabla_{x}^{j} v\right|_{L^{s}} \leq\left.\left.\left.\left. C|| x\right|^{\alpha} \nabla_{x}^{m} v\right|_{L^{p}} ^{a}|| x\right|^{\beta} v\right|_{L^{q}} ^{1-a}
$$

if and only if the following conditions hold:

$$
\frac{1}{s}+\frac{\tau-j}{n}=a\left(\frac{1}{p}+\frac{\alpha-m}{n}\right)+(1-a)\left(\frac{1}{q}+\frac{\beta}{n}\right) \quad \text { with } \quad \tau \leq a \alpha+(1-a) \beta ;
$$

if $\frac{1}{q}+\frac{\beta}{n}=\frac{1}{p}+\frac{\alpha-m}{n}$, then

$$
a(\alpha-m)+(1-a) \beta+j \leq \tau
$$

if $a=\frac{j}{m}$, then

$$
\tau=a \alpha+(1-a) \beta
$$

Corollary 2.1 For the domain $Q\left(=\Omega_{0}\right.$ or $\left.\Omega\right)$ defined in Section 1, if a function $u(y) \in C^{m}(\bar{Q})$ and

$$
\left.u\right|_{|y| \geq T} \equiv 0,
$$

where $T>0$ is a constant, $y=(t, x)$ and $|y|=\sqrt{t^{2}+|x|^{2}}$, then

(i) (2.6) still holds under the restriction (2.5) and (2.7)-(2.9), moreover, the constant $C$ on the right hand side of (2.6) is independent of $T$.

(ii) When $m=2,1<\gamma<\frac{5}{3}$ and $0<\delta<3 \gamma$, for $\mu>0$,

$$
\begin{aligned}
& \left.\left.|| y\right|^{\frac{2 \mu+2-\delta}{4}} \nabla u\right|_{L^{4}(Q)} \leq\left.\left.\left.\left. C|| y\right|^{\frac{\mu+1-\delta}{2}} \nabla^{2} u\right|_{L^{2}(Q)} ^{\frac{1}{2}}|| y\right|^{\frac{\mu+1}{2}} u\right|_{L^{\infty}(Q)} ^{\frac{1}{2}}, \\
& \left.\left.|| y\right|^{\frac{\mu+3-\delta}{2}} \nabla u\right|_{L^{4}(Q)} \leq\left.\left.\left.\left. C|| y\right|^{\frac{\mu+3-\delta}{2}} \nabla^{2} u\right|_{L^{2}(Q)} ^{\frac{1}{2}}|| y\right|^{\frac{\mu+3-\delta}{2}} u\right|_{L^{\infty}(Q)} ^{\frac{1}{2}}, \\
& \left.\left.|| y\right|^{\frac{2 \mu+2-3(\gamma-1)-\delta}{4}} \nabla u\right|_{L^{4}(Q)} \leq\left.\left.\left.\left. C|| y\right|^{\frac{\mu+1-3(\gamma-1)}{2}} \nabla^{2} u\right|_{L^{2}(Q)} ^{\frac{1}{2}}|| y\right|^{\frac{\mu+1-\delta}{2}} u\right|_{L^{\infty}(Q)} ^{\frac{1}{2}}, \\
& \left.\left.|| y\right|^{\frac{3 \gamma-3-\delta}{2}} \nabla u\right|_{L^{4}(Q)} \leq\left.\left.\left.\left. C|| y\right|^{\frac{3 \gamma-3-\delta}{2}} \nabla^{2} u\right|_{L^{2}(Q)} ^{\frac{1}{2}}|| y\right|^{\frac{3 \gamma-3-\delta}{2}} u\right|_{L^{\infty}(Q)} ^{\frac{1}{2}}, \\
& \left.\left.|| y\right|^{\frac{6 \gamma-6-\delta}{2}} \nabla u\right|_{L^{4}(Q)} \leq\left.\left.\left.\left. C|| y\right|^{\frac{6 \gamma-6-\delta}{2}} \nabla^{2} u\right|_{L^{2}(Q)} ^{\frac{1}{2}}|| y\right|^{\frac{6 \gamma-6-\delta}{2}} u\right|_{L^{\infty}(Q)} ^{\frac{1}{2}},
\end{aligned}
$$

where $\nabla=\nabla_{y}$ and $\nabla^{2}=\nabla_{y}^{2}$.

Proof. (i) The proof is completely parallel to that of Lemma 2.1 (one can check the details in [15]), then we omit it here.

(ii) In (2.5) and (2.7)-(2.9) of Lemma 2.1, set $s=4, p=2, q=\infty, a=\frac{1}{2}$ and $j=1, m=2, n=4$, one can conclude that (2.11) and (2.12) come from (2.6) with $\tau=\frac{2 \mu+2-\delta}{4}, \alpha=\frac{\mu+1-\delta}{2}, \beta=\frac{\mu+1}{2}$ and $\tau=\frac{\mu+3-\delta}{2}, \alpha=\frac{\mu+3-\delta}{2}, \beta=\frac{\mu+3-\delta}{2}$, respectively.

(2.13) and (2.14) are from (2.6) with $\tau=\frac{2 \mu+2-3(\gamma-1)-\delta}{4}, \alpha=\frac{\mu+1-3(\gamma-1)}{2}, \beta=\frac{\mu+1-\delta}{2}$ and $\tau=\alpha=\beta=\frac{3 \gamma-3-\delta}{2}$, respectively.

(2.15) is from (2.6) with $\tau=\alpha=\beta=\frac{6 \gamma-6-\delta}{2}$. And this completes the proof of the corollary.

As in [24], in order to apply Lemma 2.1 or Corollary 2.3 to derive some weighted Sobolev inequalities in $Q$ without the restriction (2.10), we need to establish an extension result as stated in 
Lemma 2.2 Set $y=(t, x), Q_{T}=\{y: 0<t<T,|x| \leq R(t)\}$ for $T>1$ and $D_{S}=\{y:(t+$ $\left.\left.\frac{1}{L}\right)^{2}+|x|^{2} \leq S^{2}, t \geq 0,|x| \leq R(t)\right\}$ for $S>1+\frac{1}{L}$. If $u(t, x) \in C^{3}\left(\bar{Q}_{T}\right)$ and $R(t)^{\beta} \nabla_{y}^{\alpha} u \in L^{2}\left(Q_{T}\right)$ $(|\alpha| \leq 3)$ with some $\beta \in \mathbb{R}$, then there exists an extension $E u \in C^{3}\left(\bar{D}_{\frac{9}{8} H}\right)$ of u such that $E u=u$ in $Q_{T},\left.E u\right|_{t \geq \frac{9}{8} H} \equiv 0$ and

$\left|R(t)^{\beta} E u\right|_{L^{\infty}\left(D_{\frac{9}{8} H}\right)} \leq C\left|R(t)^{\beta} u\right|_{L^{\infty}\left(Q_{T}\right)}, \quad\left|R(t)^{\beta} \nabla_{y}^{\alpha} E u\right|_{L^{2}\left(D_{\frac{9}{8} H}\right)} \leq C \sum_{|\nu| \leq|\alpha|}\left|R(t)^{\beta-|\alpha|+|\nu|} \nabla_{y}^{\nu} u\right|_{L^{2}\left(D_{T}\right)}$,

where $H=\sqrt{R(T)^{2}+\left(T+\frac{1}{L}\right)^{2}}$, and $C>0$ is independent of $T$.

Proof. Let $\tilde{E}$ be an extension operator defined by

$(\tilde{E} u)(t, x)= \begin{cases}u(t, x), & 0 \leq t \leq T, \quad|x| \leq R(t) ; \\ \sum_{j=1}^{4} \lambda_{j} u\left(T+\frac{j(T-t)}{4 L^{2}+4 L+1}, x\right), & T<t \leq H-\frac{1}{L}, \quad|x| \leq \sqrt{H^{2}-\left(t+\frac{1}{L}\right)^{2}},\end{cases}$

where $\sum_{j=1}^{4}\left(-\frac{j}{4 L^{2}+4 L+1}\right)^{k} \lambda_{j}=1$ for $k=0,1,2,3$.

Note that $H-\frac{1}{L}<T+L R(T)$ and $T>1$ hold. This means that for $T<t \leq H-\frac{1}{L}$ and $0 \leq j \leq 3$,

$$
T \geq T+\frac{j(T-t)}{4 L^{2}+4 L+1} \geq T-\frac{4 L R(T)}{4 L^{2}+4 L+1}>\frac{T}{4 L^{2}+4 L+1} .
$$

In addition,

$$
1 \leq \frac{t}{T+\frac{j(T-t)}{4 L^{2}+4 L+1}} \leq\left(4 L^{2}+4 L+1\right)\left(L^{2}+L+1\right) \quad \text { for } T<t \leq H-\frac{1}{L} \text { and } 0 \leq j \leq 3 .
$$

Consequently, one has that for $(t, x) \in D_{S}$,

$$
C_{1} R(t) \leq \sqrt{\left(t+\frac{1}{L}\right)^{2}+|x|^{2}} \leq C_{2} R(t) .
$$

This yields for $|\alpha| \leq 3$

$$
\left|R(t)^{\beta} \tilde{E} u\right|_{L^{\infty}\left(D_{H}\right)} \leq C\left|R(t)^{\beta} u\right|_{L^{\infty}\left(Q_{T}\right)}, \quad\left|R(t)^{\beta} \nabla_{y}^{\alpha} \tilde{E} u\right|_{L^{2}\left(D_{H}\right)} \leq C\left|R(t)^{\beta} \nabla_{y}^{\alpha} u\right|_{L^{2}\left(Q_{T}\right)} .
$$

Next, we construct the extension operator $E$ starting from the operator $\tilde{E}$. In terms of the geometric property of $D_{T}$, it is convenient to use the spherical coordinates. Let $x_{0}=t+\frac{1}{L}$, and

$$
s^{2}=\sum_{i=0}^{3} x_{i}^{2}, \quad \omega_{i}=\frac{x_{i}}{s}, \quad i=0,1,2,3 .
$$

Denoting by $\tilde{u}(s, \omega)=u(s \omega)$ with $\omega=\left(\omega_{0}, \ldots, \omega_{3}\right)$. Let $\bar{E}$ be an extension operator defined by

$$
(\bar{E} u)(s, \omega)= \begin{cases}\tilde{E} \tilde{u}(s, \omega), & (t, x) \in D_{H}, \\ \sum_{j=1}^{4} \nu_{j} \tilde{E} \tilde{u}(H+j(H-s), \omega), & (t, x) \in D_{\frac{9}{8} H} \backslash D_{H},\end{cases}
$$


where $\sum_{j=1}^{4}(-1)^{k} \nu_{j}=1$ for $k=0,1,2,3$.

Note that

$$
1 \leq \frac{s}{H+j(H-s)} \leq \frac{9}{4} \quad \text { for } H \leq s \leq \frac{9}{8} H \text { and } 0 \leq j \leq 3 .
$$

This together with (2.18) yields

$$
\left\{\begin{array}{l}
\left|R(t)^{\beta} \bar{E} u\right|_{L^{\infty}\left(D_{\frac{9}{8} H}\right)} \leq C\left|R(t)^{\beta} \tilde{E} u\right|_{L^{\infty}\left(D_{H}\right)} \leq C\left|R(t)^{\beta} u\right|_{L^{\infty}\left(Q_{T}\right)} \\
\left|R(t)^{\beta} \nabla_{t, x}^{\alpha} \bar{E} u\right|_{L^{2}\left(D_{\frac{9}{8} H}\right)} \leq C\left|R(t)^{\beta} \nabla_{t, x}^{\alpha} \tilde{E} u\right|_{L^{2}\left(D_{H}\right)} \leq C\left|R(t)^{\beta} \nabla_{t, x}^{\alpha} u\right|_{L^{2}\left(Q_{T}\right)} .
\end{array}\right.
$$

Choose a $C^{\infty}$ smooth function $\eta(s)$ with $\eta(s)=1$ for $s \leq 1$ and $\eta(s)=0$ for $s \geq \frac{9}{8}$ and set

$$
E u(t, x)=\eta\left(\frac{s}{H}\right) \bar{E} u .
$$

Then Eu satisfies (2.16), and thus Lemma 2.2 is proved.

Remark 2.1 Lemma 2.2 implies that Corollary 2.1 still holds without assumption (2.10).

The $Z$-fileds introduced in (1.14), as shown in [14], have the following properties.

\section{Lemma 2.3}

(i) $\left[Z_{1}, Z_{2}\right]=Z_{3},\left[Z_{2}, Z_{3}\right]=Z_{1},\left[Z_{3}, Z_{1}\right]=Z_{2}$.

(ii) $\left[Z_{i}, \partial_{r}\right]=0, Z_{i} r=0,\left[Z_{i}, \Delta\right]=0$.

(iii) $\nabla_{x} f \cdot \nabla_{x} g=\partial_{r} f \cdot \partial_{r} g+\frac{1}{r^{2}} \sum_{i=1}^{3} Z_{i} f \cdot Z_{i} g$ for any $C^{1}$ smooth functions $f$ and $g$.

(iv) $|Z v| \leq r\left|\nabla_{x} v\right|$ for any $C^{1}$ smooth function $v$, here and below $Z \in\left\{Z_{1}, Z_{2}, Z_{3}\right\}$.

(v) $\quad \partial_{1}=\frac{x_{1}}{r} \partial_{r}+\frac{x_{2}}{r^{2}} Z_{1}-\frac{x_{3}}{r^{2}} Z_{3} ; \quad \partial_{2}=\frac{x_{2}}{r} \partial_{r}+\frac{x_{3}}{r^{2}} Z_{2}-\frac{x_{1}}{r^{2}} Z_{1} ; \quad \partial_{3}=\frac{x_{3}}{r} \partial_{r}+\frac{x_{1}}{r^{2}} Z_{3}-\frac{x_{2}}{r^{2}} Z_{2}$.

Remark 2.2 If $u \in C^{m}\left(\mathbb{R}^{3}\right)$ with $m \in \mathbb{N}$, then by Lemma 2.3 we have $\left|\nabla_{x}^{m} u\right| \sim\left|\partial_{r}^{m} u\right|+\frac{\left|\partial_{r}^{m-1} Z u\right|}{r}+$ $\frac{\left|\partial_{r}^{m-2} Z^{2} u\right|}{r^{2}}+\ldots+\frac{\left|Z^{m} u\right|}{r^{m}}$.

As a direct consequence of Remark 2.1, we have the following weighted inequalities which will be used often in Section 5.

Lemma 2.4 Define $D_{t}=\partial_{t}+\frac{L r}{R(t)} \partial_{r}$ and $S_{0}=R(t) D_{t}$. If $1<\gamma<\frac{5}{3}, 0<\delta \leq \frac{3}{5}(\gamma-1)$, and $u(y) \in C^{4}\left(\bar{Q}_{T}\right)\left(Q_{T}=\Omega_{0} \cap\{t \leq T\}\right.$ or $\left.\Omega \cap\{t \leq T\}\right)$ with $y=(t, x)$, then there exists a generic positive constant $C$ independent of $T$ such that

$$
\begin{aligned}
& \left|R(t)^{\frac{2 \mu+2-\delta}{4}} \nabla_{y} S_{0}^{2} u\right|_{L^{4}\left(Q_{T}\right)} \leq C\left(\sum_{k=0}^{2}\left|R(t)^{\frac{\mu+1-\delta}{2}-k} \nabla_{y}^{2-k} S_{0}^{2} u\right|_{L^{2}\left(Q_{T}\right)}^{\frac{1}{2}}\right)\left|R(t)^{\frac{\mu+1}{2}} S_{0}^{2} u\right|_{L^{\infty}\left(Q_{T}\right)}^{\frac{1}{2}} ; \\
& \left|R(t)^{\frac{\mu+3-\delta}{2}} \nabla_{y}^{2} S_{0} u\right|_{L^{4}\left(Q_{T}\right)} \leq C\left(\sum_{k=0}^{2}\left|R(t)^{\frac{\mu+3-\delta}{2}-k} \nabla_{y}^{3-k} S_{0} u\right|_{L^{2}\left(Q_{T}\right)}^{\frac{1}{2}}\right)\left|R(t)^{\frac{\mu+3-\delta}{2}} \nabla_{y} S_{0} u\right|_{L^{\infty}\left(Q_{T}\right)}^{\frac{1}{2}} ;
\end{aligned}
$$




$$
\begin{aligned}
& \text { (iii) } \quad\left|R(t)^{\frac{2 \mu+5-3 \gamma-\delta}{4}} \nabla_{y} Z u\right|_{L^{4}\left(Q_{T}\right)} \leq C\left(\sum_{k=0}^{1}\left|R(t)^{\frac{\mu+4-3 \gamma}{2}-k} \nabla_{y}^{2-k} Z u\right|_{L^{2}\left(Q_{T}\right)}^{\frac{1}{2}}\right)\left|R(t)^{\frac{\mu+1-\delta}{2}} Z u\right|_{L^{\infty}\left(Q_{T}\right)}^{\frac{1}{2}} \\
& (\text { iv }) \quad\left|R(t)^{\frac{3 \gamma-3-\delta}{2}} \nabla_{y}^{2} Z u\right|_{L^{4}\left(Q_{T}\right)} \\
& \quad \leq C\left(\sum_{k=0}^{2}\left|R(t)^{\frac{3 \gamma-3-\delta}{2}-k} \nabla_{y}^{3-k} Z u\right|_{L^{2}\left(Q_{T}\right)}^{\frac{1}{2}}\right)\left|R(t)^{\frac{3 \gamma-3-\delta}{2}} \nabla_{t, x} Z u\right|_{L^{\infty}\left(Q_{T}\right)}^{\frac{1}{2}} \\
& \text { (v) } \quad\left|R(t)^{\frac{6 \gamma-6-\delta}{2}} \nabla_{y} Z D_{t} u\right|_{L^{4}\left(Q_{T}\right)} \\
& \quad \leq C\left(\sum_{k=0}^{2}\left|R(t)^{\frac{6 \gamma-6-\delta}{2}-k} \nabla_{y}^{2-k} Z D_{t} u\right|_{L^{2}\left(Q_{T}\right)}^{\frac{1}{2}}\right)\left|R(t)^{\frac{6 \gamma-6-\delta}{2}} Z D_{t} u\right|_{L^{\infty}\left(Q_{T}\right)}^{\frac{1}{2}} \\
& \text { (vi) } \quad\left|R(t)^{\frac{3 \gamma-5-\delta}{2}} \nabla_{y} Z^{2} u\right|_{L^{4}\left(Q_{T}\right)}+\left|R(t)^{\frac{3 \gamma-5-\delta}{2}} Z^{2} \nabla_{y} u\right|_{L^{4}\left(Q_{T}\right)} \\
& \quad \leq C\left(\left|R(t)^{\frac{3 \gamma-3-\delta}{2}} \nabla_{y}^{2} Z u\right|_{L^{4}\left(Q_{T}\right)}+\left|R(t)^{\frac{2 \mu+5-3 \gamma-\delta}{4}} \nabla_{y} Z u\right|_{L^{4}\left(Q_{T}\right)}\right) \\
& \text { (vii) } \quad\left|R(t)^{\frac{6 \gamma-8-\delta}{2}} Z^{2} D_{t} u\right|_{L^{4}\left(Q_{T}\right)} \leq\left|R(t)^{\frac{6 \gamma-6-\delta}{2}} \nabla_{y} Z D_{t} u\right|_{L^{4}\left(Q_{T}\right)} .
\end{aligned}
$$

Proof. Let $E$ be the extension operator given in Lemma 2.2. Then we have (i)

$$
\begin{aligned}
& \left|R(t)^{\frac{2 \mu+2-\delta}{4}} \nabla_{y} S_{0}^{2} u\right|_{L^{4}\left(Q_{T}\right)} \\
\leq & C\left|R(t)^{\frac{2 \mu+2-\delta}{4}} \nabla_{y} E\left(S_{0}^{2} u\right)\right|_{L^{4}\left(Q_{T}\right)} \\
\leq & \left.C\left|R(t)^{\frac{\mu+1-\delta}{2}} \nabla_{y}^{2} E\left(S_{0}^{2} u\right)\right|_{L^{2}\left(Q_{T}\right)}^{\frac{1}{2}}\left|R(t)^{\frac{\mu+1}{2}} E\left(S_{0}^{2} u\right)\right|_{L^{\infty}\left(Q_{T}\right)}^{\frac{1}{2}} \quad \text { (Applying (2.11) for } E\left(S_{0}^{2} u\right)\right) \\
\leq & C\left(\sum_{k=0}^{2}\left|R(t)^{\frac{\mu+1-\delta}{2}-k} \nabla_{y}^{2-k} S_{0}^{2} u\right|_{L^{2}\left(Q_{T}\right)}^{\frac{1}{2}}\right)\left|R(t)^{\frac{\mu+1}{2}} S_{0}^{2} u\right|_{L^{\infty}\left(Q_{T}\right)}^{\frac{1}{2}} \quad \quad \quad \text { (By Lemma 2.2) }
\end{aligned}
$$

(ii)

$$
\begin{aligned}
& \left|R(t)^{\frac{\mu+3-\delta}{2}} \nabla_{y}^{2} S_{0} u\right|_{L^{4}\left(Q_{T}\right)} \\
\leq & C\left|R(t)^{\frac{\mu+3-\delta}{2}} \nabla_{y} E\left(\nabla_{y} S_{0} u\right)\right|_{L^{4}\left(Q_{T}\right)} \\
\leq & \left|R(t)^{\frac{\mu+3-\delta}{2}} \nabla_{y}^{2} E\left(\nabla_{y} S_{0} u\right)\right|_{L^{2}\left(Q_{T}\right)}^{\frac{1}{2}}\left|R(t)^{\frac{\mu+3-\delta}{2}} E\left(\nabla_{y} S_{0} u\right)\right|_{L^{\infty}\left(Q_{T}\right)}^{\frac{1}{2}} \quad\left(\text { Applying (2.12) for } E\left(\nabla_{y} S_{0} u\right)\right) \\
\leq & C\left(\sum_{k=0}^{2}\left|R(t)^{\frac{\mu+3-\delta}{2}-k} \nabla_{y}^{3-k} S_{0} u\right|_{L^{2}\left(Q_{T}\right)}^{\frac{1}{2}}\right)\left|R(t)^{\frac{\mu+3-\delta}{2}} \nabla_{y} S_{0} u\right|_{L^{\infty}\left(Q_{T}\right)}^{\frac{1}{2}} \quad \quad \quad \text { (By Lemma 2.2) }
\end{aligned}
$$

(iii)

$$
\begin{aligned}
& \left|R(t)^{\frac{2 \mu+5-3 \gamma-\delta}{4}} \nabla_{y} Z u\right|_{L^{4}\left(Q_{T}\right)} \\
\leq & C\left|R(t)^{\frac{2 \mu+5-3 \gamma-\delta}{4}} \nabla_{y} E(Z u)\right|_{L^{4}\left(Q_{T}\right)} \\
\leq & C\left|R(t)^{\frac{\mu+4-3 \gamma}{2}} \nabla_{y}^{2} E(Z u)\right|_{L^{2}\left(Q_{T}\right)}^{\frac{1}{2}}\left|R(t)^{\frac{\mu+1-\delta}{2}} E(Z u)\right|_{\left.L^{\infty}\left(Q_{T}\right) \quad \quad \quad \text { Applying (2.13) for } E(Z u)\right)}^{\frac{1}{2}} \\
\leq & C\left(\sum_{k=0}^{1}\left|R(t)^{\frac{\mu+4-3 \gamma}{2}-k} \nabla_{y}^{2-k} Z u\right|_{L^{2}\left(Q_{T}\right)}^{\frac{1}{2}}\right)\left|R(t)^{\frac{\mu+1-\delta}{2}} Z u\right|_{L^{\infty}\left(Q_{T}\right)}^{\frac{1}{2}} \quad \quad \text { (By Lemma 2.2) }
\end{aligned}
$$


(iv)

$$
\begin{aligned}
& \left|R(t)^{\frac{3 \gamma-3-\delta}{2}} \nabla_{y}^{2} Z u\right|_{L^{4}\left(Q_{T}\right)} \\
\leq & \left|R(t)^{\frac{3 \gamma-3-\delta}{2}} \nabla_{y} E\left(\nabla_{y} Z u\right)\right|_{L^{4}\left(Q_{T}\right)} \\
\leq & \left.C\left|R(t)^{\frac{3 \gamma-3-\delta}{2}} \nabla_{y}^{2} E\left(\nabla_{y} Z u\right)_{L^{2}\left(Q_{T}\right)}^{\frac{1}{2}}\right| R(t)^{\frac{3 \gamma-3-\delta}{2}} E\left(\nabla_{y} Z u\right)\right|_{\left.L^{\infty}\left(Q_{T}\right) \quad \quad \text { Applying (2.14) for } E\left(\nabla_{y} Z u\right)\right)} ^{\frac{1}{2}} \leq \quad C\left(\sum_{k=0}^{2}\left|R(t)^{\frac{3 \gamma-3-\delta}{2}-k} \nabla_{y}^{3-k} Z u\right|_{L^{2}\left(Q_{T}\right)}^{\frac{1}{2}}\right)\left|R(t)^{\frac{3 \gamma-3-\delta}{2}} \nabla_{y} Z u\right|_{L^{\infty}\left(Q_{T}\right)}^{\frac{1}{2}} \quad \quad \text { (By Lemma 2.2) }
\end{aligned}
$$

(v)

$$
\begin{aligned}
& \left|R(t)^{\frac{6 \gamma-6-\delta}{2}} \nabla_{y} Z D_{t} u\right|_{L^{4}\left(Q_{T}\right)} \\
\leq & C\left|R(t)^{\frac{6 \gamma-6-\delta}{2}} \nabla_{y} E\left(Z D_{t} u\right)\right|_{L^{4}\left(Q_{T}\right)} \\
\leq & \left.C\left|R(t)^{\frac{6 \gamma-6-\delta}{2}} \nabla_{y}^{2} E\left(Z D_{t} u\right)\right|_{L^{2}\left(Q_{T}\right)}^{\frac{1}{2}}\left|R(t)^{\frac{6 \gamma-6-\delta}{2}} E\left(Z D_{t} u\right)\right|_{L^{\infty}\left(Q_{T}\right)}^{\frac{1}{2}} \quad \text { (Applying (2.15) for } E\left(Z D_{t} u\right)\right) \\
\leq & C\left(\sum_{k=0}^{2}\left|R(t)^{\frac{6 \gamma-6-\delta}{2}}-k \nabla_{y}^{2-k} Z D_{t} u\right|_{L^{2}\left(Q_{T}\right)}^{\frac{1}{2}}\right)\left|R(t)^{\frac{6 \gamma-6-\delta}{2}} Z D_{t} u\right|_{L^{\infty}\left(Q_{T}\right)}^{\frac{1}{2}} \quad \quad \text { (By Lemma 2.2) }
\end{aligned}
$$

(vi) and (vii) follow from the definition of $Z$-fields in (1.14) and Lemma 2.3.

\section{Reformulation of problem (1.10) with (1.16) and (1.17)}

Firstly, we state a local solvability result on problem (1.10) with (1.11) and (1.17).

Lemma 3.1 There exists a $T_{0}>0$ such that the problem (1.10) with (1.16) and (1.17) has a local solution $\Phi(t, x) \in C\left(\left[0, T_{0}\right], H^{5}\left(S_{t}\right)\right) \cap C^{1}\left(\left[0, T_{0}\right], H^{4}\left(S_{t}\right)\right)$ with $S_{t}=\{x:|x| \leq R(t)\}$. Moreover,

$$
\|\Phi(t, x)-\hat{\Phi}(t, r)\|_{C\left(\left[0, T_{0}\right], H^{5}\left(S_{t}\right)\right)}+\|\Phi(t, x)-\hat{\Phi}(t, r)\|_{C^{1}\left(\left[0, T_{0}\right], H^{4}\left(S_{t}\right)\right)} \leq C \varepsilon,
$$

where $\hat{\Phi}(t, r)$ is given in (2.4).

Proof. The quasilinear equation (1.10) is strictly hyperbolic with respect to $t$. Thus, by the standard Picard iteration as in [17], one can derive that Lemma 3.1 holds.

Next, we reformulate (1.10) with (1.16)-(1.17).

Let $\dot{\Phi}=\Phi-\hat{\Phi}$. Then (1.10) can be reduced to

$$
\mathcal{L} \dot{\Phi}=\dot{f} \quad \text { in } \Omega
$$

where

$$
\left\{\begin{aligned}
& \mathcal{L} \dot{\Phi}= \partial_{t}^{2} \dot{\Phi}+2 \sum_{i=1}^{3} \partial_{i} \hat{\Phi} \partial_{t i}^{2} \dot{\Phi}+\sum_{i, j=1}^{3} \partial_{i} \hat{\Phi} \partial_{j} \hat{\Phi} \partial_{i j}^{2} \dot{\Phi}+2 \sum_{i=1}^{3} \partial_{t i}^{2} \hat{\Phi} \partial_{i} \dot{\Phi}-\hat{c}^{2} \Delta \dot{\Phi} \\
&+\frac{3 L(\gamma-1)}{R(t)}\left(\partial_{t} \dot{\Phi}+\sum_{i=1}^{3} \partial_{i} \hat{\Phi} \partial_{i} \dot{\Phi}\right), \\
& \dot{f}=\sum_{i=1}^{3} f_{0 i} \partial_{t i}^{2} \dot{\Phi}+\sum_{1 \leq i \neq j \leq 3} f_{i j} \partial_{i j}^{2} \dot{\Phi}+\sum_{i=1}^{3} f_{i i} \partial_{i}^{2} \dot{\Phi}+f_{0},
\end{aligned}\right.
$$


with

$$
\left\{\begin{array}{l}
f_{0 i}=-2 \partial_{i} \dot{\Phi} \\
f_{i j}=-\partial_{i} \dot{\Phi} \partial_{j} \dot{\Phi}-2 \partial_{i} \hat{\Phi} \partial_{j} \dot{\Phi} \\
f_{i i}=-\left(\partial_{i} \dot{\Phi}\right)^{2}-(\gamma-1)\left(\partial_{t} \dot{\Phi}+\sum_{j=1}^{3} \partial_{j} \hat{\Phi} \partial_{j} \dot{\Phi}+\frac{1}{2} \sum_{j=1}^{3}\left(\partial_{j} \dot{\Phi}\right)^{2}\right)-2 \partial_{i} \hat{\Phi} \partial_{i} \dot{\Phi} \\
f_{0}=-\frac{(3 \gamma-1) L}{2 R(t)} \sum_{i=1}^{3}\left(\partial_{i} \dot{\Phi}\right)^{2}
\end{array}\right.
$$

For later analysis, we use $Z$-fields to rewrite $\mathcal{L} \dot{\Phi}$ as follows:

$$
\begin{aligned}
\mathcal{L} \dot{\Phi}= & \partial_{t}^{2} \dot{\Phi}+2 \partial_{r} \hat{\Phi} \partial_{t r}^{2} \dot{\Phi}+\left(\left(\partial_{r} \hat{\Phi}\right)^{2}-\hat{c}^{2}\right) \partial_{r}^{2} \dot{\Phi}-\frac{\hat{c}^{2}}{r^{2}} \sum_{i=1}^{3} Z_{i}^{2} \dot{\Phi}+\frac{\gamma-1}{r}\left(r \partial_{r}^{2} \hat{\Phi}+2 \partial_{r} \hat{\Phi}\right) \partial_{t} \dot{\Phi} \\
& +\frac{1}{r}\left(2 r \partial_{t r}^{2} \hat{\Phi}+(\gamma+1) r \partial_{r} \hat{\Phi} \partial_{r}^{2} \hat{\Phi}+2(\gamma-1)\left(\partial_{r} \hat{\Phi}\right)^{2}-2 \hat{c}^{2}\right) \partial_{r} \dot{\Phi}
\end{aligned}
$$

On the lateral boundary $\partial \Omega$ of $\Omega, \dot{\Phi}$ satisfies

$$
\partial_{r} \dot{\Phi}=0
$$

In addition, we have the following initial data of $\dot{\Phi}$ from (1.17)

$$
\dot{\Phi}(0, x)=\varepsilon \Phi_{0}(x), \quad \partial_{t}(0, x)=\varepsilon \Phi_{1}(x) .
$$

Remark 3.1. By (2.3) or (2.4), we know that $\mathcal{L} \dot{\Phi}$ in (3.2) has the following form at the $t$-axis:

$$
\partial_{t}^{2} \dot{\Phi}-\frac{\gamma}{R^{3(\gamma-1)}(t)}\left(\partial_{1}^{2}+\partial_{2}^{2}+\partial_{3}^{2}\right) \dot{\Phi}+\frac{3 L(\gamma-1)}{R(t)} \partial_{t} \dot{\Phi}
$$

which is strictly hyperbolic but degenerate as $t \rightarrow \infty$. On the other hand, the operator $\mathcal{L}=\partial_{t}^{2}+$ $\frac{2 L r}{R(t)} \partial_{t r}^{2}+\sum_{i, j=1}^{3} \frac{L^{2} x_{i} x_{j}}{R^{2}(t)} \partial_{i j}^{2}-\frac{\gamma}{R^{3(\gamma-1)}(t)} \Delta+\frac{3 L(\gamma-1)}{R(t)}\left(\partial_{t}+\frac{L r}{R(t)} \partial_{r}\right)$ in (3.2) is different from [24], where the corresponding linear operator is $\partial_{t}^{2}-\frac{1}{R^{2(\gamma-1)}(t)}\left(\partial_{1}^{2}+\partial_{2}^{2}\right)+\frac{2 L(\gamma-1)}{R(t)} \partial_{t}$. Recently, with respect to the semilinear wave equations

$$
\partial_{t}^{2} u-\Delta u+\frac{\mu}{(1+t)^{\alpha}} \partial_{t} u=f(u)
$$

where $\mu>0$ and $\alpha>0$ are suitable constants, there have been extensive works on the global existence or blowup results for different nonlinear function $f(u), c f$. [9], [21-22] and the references therein. However, for the critical exponent $\alpha=1$ and near the critical value $\mu=1$, there are still some open questions on the blowup or global existence of solutions to (3.7). Here, (3.6) corresponds to the critical case of (3.7) when $L=1$ and $\gamma$ is close to $\frac{4}{3}$. 


\section{The first-order weighted energy estimate and reformulation of (3.2)- (3.3)}

In this section, we derive the weighted energy estimate of $\nabla_{t, x} \dot{\Phi}$ for the linear part (3.3) together with (3.4)-(3.5).

Set $\Omega_{T}=\Omega \cap\{0<t<T\}$ and $B_{T}=\partial \Omega \cap\{0<t<T\}$. Then, we have

Theorem 4.1 Let $\dot{\Phi} \in C^{2}\left(\bar{\Omega}_{T}\right)$ satisfy the boundary condition (3.4) and initial data condition (3.5). Then for $0<\gamma<\frac{5}{3}$, there exists a multiplier $\mathcal{M} \dot{\Phi}=R(t)^{\mu} a(t) D_{t} \dot{\Phi}$ such that for fixed constant $\mu=6 \gamma-9$ we have

$$
\begin{aligned}
& R(T)^{\mu} \int_{S_{T}}\left(D_{t} \dot{\Phi}\right)^{2} d S+T^{\mu-3(\gamma-1)} \int_{S_{T}}\left(\nabla_{x} \dot{\Phi}\right)^{2} d S \\
& \quad+C \int_{\Omega_{T}}\left(R(t)^{\mu-1-\delta}\left(D_{t} \dot{\Phi}\right)^{2}+R(t)^{\mu-1-3(\gamma-1)}\left(\nabla_{x} \dot{\Phi}\right)^{2}\right) d t d x \\
& \leq \int_{\Omega_{T}} \mathcal{L} \dot{\Phi} \cdot \mathcal{M} \dot{\Phi} d t d x+C \varepsilon^{2},
\end{aligned}
$$

where $D_{t}=\partial_{t}+\sum_{i=1}^{3} \partial_{i} \hat{\Phi} \partial_{i}=\partial_{t}+\frac{L r}{R(t)} \partial_{r}$ is the material derivative, $C>0$ is a generic positive constant depending only on the initial data, and $\delta>0$ is a small fixed constant.

Remark 4.1 The choice of $\mu=6 \gamma-9$ in (4.1) is necessary because of the following two reasons: First, to guarantee the positivity of III in (4.2), $\mu \leq 6 \gamma-9$; Second, by the Bernoulli law (1.7), we have $c^{2}(\rho)=c^{2}(\hat{\rho})-(\gamma-1) D_{t} \dot{\Phi}-\frac{\gamma-1}{2}\left|\nabla_{x} \dot{\Phi}\right|^{2}$. Notice that only the estimate of $\left|D_{t} \dot{\Phi}\right| \leq C \varepsilon R(t)^{-\frac{\mu+3}{2}}$ and $\left|\nabla_{x} \dot{\Phi}\right| \leq C \varepsilon R(t)^{-\frac{\mu-3(\gamma-1)+3}{2}}$ can be obtained as shown in Sections 5 and 6 . On the other hand, $c^{2}(\hat{\rho}(t))=\gamma R(t)^{-3(\gamma-1)}$ holds. Therefore, in order to guarantee the absence of vacuum for any finite time $t$ in $\Omega$, we need to choose the constant $\mu$ such that $-\frac{\mu+3}{2} \leq-3(\gamma-1)$, that is, $\mu \geq 6 \gamma-9$. In combination, $\mu=6 \gamma-9$.

Proof. Choosing $\mathcal{M} \dot{\Phi}=R(t)^{\mu}\left(a(t, r) \partial_{t} \dot{\Phi}+b(t, r) \partial_{r} \dot{\Phi}\right)$, where the non-negative functions $a(t, r)$ and $b(t, r)$ will be determined later, then

$$
\int_{\Omega_{T}} \mathcal{L} \dot{\Phi} \cdot \mathcal{M} \dot{\Phi} d t d x=I+I I+I I I
$$

where

$$
\begin{aligned}
& I=\int_{B_{T}} \frac{R(t)^{\mu}}{2 \sqrt{1+L^{2}}}(L a(t, r)-b(t, r))\left(\left(\partial_{t} \dot{\Phi}\right)^{2}-\frac{\hat{c}^{2}}{r^{2}} \sum_{i=1}^{3}\left(Z_{i} \dot{\Phi}\right)^{2}\right. \\
& \left.\quad+2\left(\left(L^{2}-\hat{c}^{2}\right) a(t, r)-L b(t, r)\right) \partial_{t} \dot{\Phi} \partial_{r} \dot{\Phi}+\left(L\left(L^{2}-\hat{c}^{2}\right) a(t, r)-\left(L^{2}+\hat{c}^{2}\right) b(t, r)\right)\left(\partial_{r} \dot{\Phi}\right)^{2}\right) d S \\
& I I \equiv I I_{1}-I I_{2}, \\
& I I I=\int_{\Omega_{T}}\left(A(t, r)\left(\partial_{t} \dot{\Phi}\right)^{2}+B(t, r) \partial_{t} \dot{\Phi} \partial_{r} \dot{\Phi}+C(t, r)\left(\partial_{r} \dot{\Phi}\right)^{2}+\frac{1}{r^{2}} D(t, r) \sum_{i=1}^{3}\left(Z_{i} \dot{\Phi}\right)^{2}\right) d t d x,
\end{aligned}
$$


with

$$
\begin{aligned}
I I_{1}= & \int_{S_{T}} R(t)^{\mu}\left(\frac{1}{2} a(t, r)\left(\partial_{t} \dot{\Phi}\right)^{2}+b(t, r) \partial_{t} \dot{\Phi} \partial_{r} \dot{\Phi}+\left(b(t, r) \partial_{r} \hat{\Phi}-\frac{1}{2}\left(\left(\partial_{r} \hat{\Phi}\right)^{2}-\hat{c}^{2}\right) a(t, r)\right)\left(\partial_{r} \dot{\Phi}\right)^{2}\right. \\
& \left.+\frac{\hat{c}^{2}}{2 r^{2}} a(t, r) \sum_{i=1}^{3}\left(Z_{i} \dot{\Phi}\right)^{2}\right) d S \\
I I_{2}= & \int_{S^{0}}\left(\frac{1}{2} a(t, r)\left(\partial_{t} \dot{\Phi}\right)^{2}+b(t, r) \partial_{t} \dot{\Phi} \partial_{r} \dot{\Phi}+\left(b(t, r) \partial_{r} \hat{\Phi}-\frac{1}{2}\left(\left(\partial_{r} \hat{\Phi}\right)^{2}-\hat{c}^{2}\right) a(t, r)\right)\left(\partial_{r} \dot{\Phi}\right)^{2}\right. \\
& \left.+\frac{\hat{c}^{2}}{2 r^{2}} a(t, r) \sum_{i=1}^{3}\left(Z_{i} \dot{\Phi}\right)^{2}\right) d S, \\
A(t, r) & =-\frac{1}{2} \partial_{t}\left(R(t)^{\mu} a(t, r)\right)-R(t)^{\mu} \partial_{r}\left(a(t, r) \partial_{r} \hat{\Phi}\right)-2 R(t)^{\mu} r^{-1} a(t, r) \partial_{r} \hat{\Phi} \\
& +r^{-2} \partial_{r}\left(\frac{1}{2} r^{2} R(t)^{\mu} b(t, r)\right)+(\gamma-1)\left(\partial_{r}^{2} \hat{\Phi}+\frac{2}{r} \partial_{r} \hat{\Phi}\right) R(t)^{\mu} a(t, r), \\
B(t, r) & =-R(t)^{\mu} \partial_{r}\left(\left(\left(\partial_{r} \hat{\Phi}\right)^{2}-\hat{c}^{2}\right) a(t, r)\right)-\frac{2}{r} R(t)^{\mu} a(t, r)\left(\left(\partial_{r} \hat{\Phi}\right)^{2}-\hat{c}^{2}\right)-\partial_{t}\left(R(t)^{\mu} b(t, r)\right) \\
& +R(t)^{\mu} a(t, r)\left(2 \partial_{t r} \hat{\Phi}+(\gamma+1) \partial_{r} \hat{\Phi} \partial_{r}^{2} \hat{\Phi}+\frac{2(\gamma-1)}{r}\left(\partial_{r} \hat{\Phi}\right)^{2}-\frac{2}{r} \hat{c}^{2}\right) \\
& +(\gamma-1)\left(\partial_{r}^{2} \hat{\Phi}+\frac{2}{r} \partial_{r} \hat{\Phi}\right) R(t)^{\mu} b(t, r), \\
D(t, r) & =-\frac{1}{2} \partial_{t}\left(\hat{c}^{2} R(t)^{\mu} a(t, r)\right)-\partial_{r}\left(\frac{1}{2} R(t)^{\mu} b(t, r) \hat{c}^{2}\right) . \\
& -\partial_{t}\left(R(t)^{\mu} b(t, r) \partial_{r} \hat{\Phi}\right)+R(t)^{\mu} b(t, r)\left(2 \partial_{t r}^{2} \hat{\Phi}+(\gamma+1) \partial_{r} \hat{\Phi} \partial_{r}^{2} \hat{\Phi}+\frac{2(\gamma-1)}{r}\left(\partial_{r} \hat{\Phi}\right)^{2}-\frac{2}{r} \hat{c}^{2}\right) \\
& =\frac{1}{2} \partial_{t}\left(\left(\left(\partial_{r} \hat{\Phi}\right)^{2}-\hat{c}^{2}\right) R(t)^{\mu} a(t, r)\right)-r^{-2} \partial_{r}\left(\frac{1}{2} R(t)^{\mu} b(t, r) r^{2}\left(\left(\partial_{r} \hat{\Phi}\right)^{2}-\hat{c}^{2}\right)\right)
\end{aligned}
$$

In view of the boundary condition (3.4), we have

$$
I=\int_{B_{T}} \frac{1}{2 \sqrt{1+L^{2}}}(L a(t, R(t))-b(t, R(t)))\left(\left(\partial_{t} \dot{\Phi}\right)^{2}-\frac{\hat{c}^{2}}{r^{2}} \sum_{i=1}^{3}\left(Z_{i} \dot{\Phi}\right)^{2}\right) d S .
$$

To guarantee $I \geq 0$, it requires that on the boundary $r=R(t)$,

$$
b(t, R(t))=L a(t, R(t)) .
$$

In this case,

$$
I=0 .
$$

Next, we consider $I I$. To fulfill $I I_{1}>0$, it requires on $t=T$

$$
\left\{\begin{array}{l}
a(t, r)>0 \\
b(t, r)^{2}-a(t, r)\left(2 b(t, r) \partial_{r} \hat{\Phi}-\left(\left(\partial_{r} \hat{\Phi}\right)^{2}-\hat{c}^{2}\right) a(t, r)\right) \leq 0
\end{array}\right.
$$


This means that on $\{t=T\}$,

$$
\left\{\begin{array}{l}
a(t, r)>0 \\
\partial_{r} \hat{\Phi}-\hat{c} \leq \frac{b(t, r)}{a(t, r)} \leq \partial_{r} \hat{\Phi}+\hat{c}
\end{array}\right.
$$

Thus, combining (4.3) and (4.6) yields

$$
b(t, r)=\partial_{r} \hat{\Phi} \cdot a(t, r) .
$$

On the other hand, by $\hat{c}^{2}=\gamma R(t)^{-2(\gamma-1)}$, we have

$$
\begin{aligned}
I I_{1} & =\int_{S_{T}} \frac{1}{2} R(t)^{\mu} a(T, r)\left(\left(\partial_{t} \dot{\Phi}+\partial_{r} \hat{\Phi} \partial_{r} \dot{\Phi}\right)^{2}+\hat{c}^{2}\left(\left(\partial_{r} \dot{\Phi}\right)^{2}+\frac{1}{r^{2}} \sum_{i=1}^{3}\left(Z_{i} \dot{\Phi}\right)^{2}\right)\right) d S \\
& \geq C \int_{S_{T}} a(T, r)\left(R(T)^{\mu}\left(D_{t} \dot{\Phi}\right)^{2}+R(T)^{\mu-3(\gamma-1)}\left(\left(\partial_{r} \dot{\Phi}\right)^{2}+\frac{1}{r^{2}} \sum_{i=1}^{3}\left(Z_{i} \dot{\Phi}\right)^{2}\right)\right) d S .
\end{aligned}
$$

It follows from Lemma 3.1 that

$$
I I_{2} \leq C \varepsilon^{2}
$$

Finally, we deal with $I I I$. In fact, we only need to choose $a(t, r) \equiv a(t)$. In this case, direct computation yields

$$
\left\{\begin{aligned}
A(t, r)= & \frac{1}{2} R(t)^{\mu-1}\left(L(6 \gamma-9-\mu) a(t)-R(t) a^{\prime}(t)\right) \\
B(t, r)= & L r R(t)^{\mu-2}\left(L(6 \gamma-9-\mu) a(t)-R(t) a^{\prime}(t)\right) \\
C(t, r)= & \frac{1}{2} R(t)^{\mu-3 \gamma-3}\left(\left(L \gamma(-4+3 \gamma-\mu) R(t)^{5}+L^{2} r^{2} R(t)^{3 \gamma}(-9+6 \gamma-\mu)\right) a(t)\right. \\
& \left.\quad-\left(L^{2} r^{2} R(t)^{1+3 \gamma}+\gamma R(t)^{6}\right) a^{\prime}(t)\right) \\
D(t, r)= & \frac{\gamma}{2} R(t)^{\mu-3 \gamma+2}\left(L(3 \gamma-4-\mu) a(t)-R(t) a^{\prime}(t)\right)
\end{aligned}\right.
$$

In order to have $I I I>0$, we choose

$$
A(t, r)>0, \quad B(t, r)^{2}-4 A(t, r) C(t, r)<0, \quad D(t, r)>0 .
$$

From this, we naturally set

$$
\mu=6 \gamma-9, \quad a(t)>0 \quad \text { and } \quad a^{\prime}(t)<0 .
$$

If we choose

$$
a(t)=1+R(t)^{-\delta} \quad \text { with } \quad \delta>0,
$$

then

$$
\begin{aligned}
I I I=\int_{\Omega_{T}} & \left\{\frac{\delta}{2} R(t)^{\mu-1-\delta}\left(\partial_{t} \dot{\Phi}+\partial_{r} \hat{\Phi} \partial_{r} \dot{\Phi}\right)^{2}\right. \\
& \left.+\frac{\gamma}{2} R(t)^{\mu-1-3(\gamma-1)}\left(L(5-3 \gamma) a(t)-R(t) a^{\prime}(t)\right)\left(\left(\partial_{r} \dot{\Phi}\right)^{2}+\frac{1}{r^{2}} \sum_{i=1}^{3}\left(Z_{i} \dot{\Phi}\right)^{2}\right)\right\} d t d x
\end{aligned}
$$




$$
\geq C \int_{\Omega_{T}}\left\{R(t)^{\mu-1-\delta}\left(D_{t} \dot{\Phi}\right)^{2}+R(t)^{\mu-1-3(\gamma-1)}\left(\left(\partial_{r} \dot{\Phi}\right)^{2}+\frac{1}{r^{2}} \sum_{i=1}^{3}\left(Z_{i} \dot{\Phi}\right)^{2}\right)\right\} d t d x .
$$

Substituting (4.4), (4.8)-(4.9) and (4.13) into (4.2) yields

$$
\begin{aligned}
& R(T)^{\mu} \int_{S_{T}}\left(D_{t} \dot{\Phi}\right)^{2} d S+R(T)^{\mu-3(\gamma-1)} \int_{S_{T}}\left(\left(\partial_{r} \dot{\Phi}\right)^{2}+\frac{1}{r^{2}} \sum_{i=1}^{3}\left(Z_{i} \dot{\Phi}\right)^{2}\right) d S \\
& \quad+C \int_{\Omega_{T}}\left(R(t)^{\mu-1-\delta}\left(D_{t} \dot{\Phi}\right)^{2}+R(t)^{\mu-1-3(\gamma-1)}\left(\left(\partial_{r} \dot{\Phi}\right)^{2}+\frac{1}{r^{2}} \sum_{i=1}^{3}\left(Z_{i} \dot{\Phi}\right)^{2}\right)\right) d t d x \\
& \leq \int_{\Omega_{T}} \mathcal{L} \dot{\Phi} \cdot \mathcal{M} \dot{\Phi} d t d x+C \varepsilon^{2} .
\end{aligned}
$$

This together with Remark 2.2 give (4.1), and it completes the proof of the lemma.

Note that the material derivative $D_{t}$ plays a crucial role in the energy estimate (4.1). Then it is necessary that we rewrite equation (3.2) and formula (3.3) by using the operator $D_{t}$ as follows:

$$
\left\{\begin{array}{l}
\mathcal{L} \dot{\Phi}=D_{t}^{2} \dot{\Phi}-\hat{c}^{2} \Delta \dot{\Phi}+\frac{3 L(\gamma-1)}{R(t)} D_{t} \dot{\Phi} \\
\dot{f}=\sum_{i=1}^{3} \tilde{f}_{0 i} \partial_{i} D_{t} \dot{\Phi}+\sum_{1 \leq i \neq j \leq 3} \tilde{f}_{i j} \partial_{i j}^{2} \dot{\Phi}+\sum_{i=1}^{3} \tilde{f}_{i i} \partial_{i}^{2} \dot{\Phi}+\tilde{f}_{0}
\end{array}\right.
$$

with

$$
\left\{\begin{array}{l}
\tilde{f}_{0 i}=-2 \partial_{i} \dot{\Phi} \\
\tilde{f}_{i j}=-\partial_{i} \dot{\Phi} \partial_{j} \dot{\Phi} \\
\tilde{f}_{i i}=-\left(\partial_{i} \dot{\Phi}\right)^{2}-(\gamma-1)\left(D_{t} \dot{\Phi}+\frac{1}{2} \sum_{j=1}^{3}\left(\partial_{j} \dot{\Phi}\right)^{2}\right) \\
\tilde{f}_{0}=-\frac{(3 \gamma-5) L}{2 R(t)} \sum_{i=1}^{3}\left(\partial_{i} \dot{\Phi}\right)^{2}
\end{array}\right.
$$

Especially, in the spherical coordinates, (4.15) has the form

$$
\mathcal{L} \dot{\Phi}=D_{t}^{2} \dot{\Phi}-\hat{c}^{2}\left(\partial_{r}^{2} \dot{\Phi}+\frac{1}{r^{2}} \sum_{i=1}^{3} Z_{i}^{2} \dot{\Phi}+\frac{2}{r} \partial_{r} \dot{\Phi}\right)+\frac{3 L(\gamma-1)}{R(t)} D_{t} \dot{\Phi}=\dot{f},
$$

where

$$
\begin{aligned}
\dot{f} & =-2 \partial_{r} \dot{\Phi} D_{t} \partial_{r} \dot{\Phi}+\frac{2}{r^{2}} \sum_{i=1}^{3} Z_{i} \dot{\Phi} D_{t} Z_{i} \dot{\Phi}-\left((\gamma-1) D_{t} \dot{\Phi}+\frac{\gamma+1}{2}\left(\partial_{r} \dot{\Phi}\right)^{2}+\frac{\gamma-1}{2 r^{2}} \sum_{i=1}^{3}\left(Z_{i} \dot{\Phi}\right)^{2}\right) \partial_{r}^{2} \dot{\Phi} \\
& -\frac{2}{r^{2}} \partial_{r} \dot{\Phi} \sum_{i=1}^{3} Z_{i} \dot{\Phi} \partial_{r} Z_{i} \dot{\Phi}-\frac{\gamma-1}{r^{2}}\left(D_{t} \dot{\Phi}+\frac{1}{2}\left(\partial_{r} \dot{\Phi}\right)^{2}+\frac{1}{2 r^{2}} \sum_{i=1}^{3}\left(Z_{i} \dot{\Phi}\right)^{2}\right) \sum_{i=1}^{3} Z_{i}^{2} \dot{\Phi} \\
& -\frac{1}{r^{4}} \sum_{i, j=1}^{3} Z_{i} \dot{\Phi} Z_{j} \dot{\Phi} Z_{i} Z_{j} \dot{\Phi}-\frac{2(\gamma-1)}{r} \partial_{r} \dot{\Phi} D_{t} \dot{\Phi}-\frac{(3 \gamma-1) L}{2 R(t)}\left(\partial_{r} \dot{\Phi}\right)^{2}-\frac{3(\gamma-1) L}{2 r^{2} R(t)} \sum_{i=1}^{3}\left(Z_{i} \dot{\Phi}\right)^{2} \\
& +\frac{\gamma-1}{r}\left(\left(\partial_{r} \dot{\Phi}\right)^{2}-\frac{1}{r^{2}} \sum_{i=1}^{3}\left(Z_{i} \dot{\Phi}\right)^{2}\right)-\sum_{i, j=1}^{3} \frac{C_{i j}}{r^{3}}\left(\frac{L r}{R(t)}+\partial_{r} \dot{\Phi}\right) Z_{i} \dot{\Phi} Z_{j} \dot{\Phi}-\sum_{i, j, k=1}^{3} \frac{C_{i j k}}{r^{4}} Z_{i} \dot{\Phi} Z_{j} \dot{\Phi} Z_{k} \dot{\Phi} .
\end{aligned}
$$


Here, we point out that the precise expression of $\dot{f}$ is useful in the higher order energy estimates of $\dot{\Phi}$, cf. Setion 5.

\section{Higher order weighted energy estimates of $\dot{\Phi}$}

In this section, we will derive the higher order energy estimates of $\dot{\Phi}$ to (3.1) with (3.4)-(3.5). For this, we need to take care of the difficulties coming from the Neumann boundary condition (3.4), the asymptotic degeneracy of some coefficients in (4.16), and the different decay rates of $D_{t} \dot{\Phi}$ and $\nabla_{x} \dot{\Phi}$.

Theorem 5.1 Let $\dot{\Phi} \in C^{4}\left(\bar{\Omega}_{T}\right)$ be the solution to (3.1) with (3.4)-(3.5), and assume with some $\delta>0$,

$$
\left\{\begin{array}{l}
\left|\nabla_{x} \dot{\Phi}\right| \leq M \varepsilon R(t)^{-3(\gamma-1)+\frac{\delta}{2}}, \quad\left|R(t)^{l-1} D_{t}^{l} \dot{\Phi}\right| \leq M \varepsilon R(t)^{-3(\gamma-1)}, \quad \text { for } l=1,2, \\
\left|R(t) \nabla_{x} D_{t} \dot{\Phi}\right| \leq M \varepsilon R(t)^{-3(\gamma-1)+\frac{\delta}{2}}, \quad\left|R(t) \nabla_{x}^{2} \dot{\Phi}\right| \leq M \varepsilon R(t)^{-\frac{3(\gamma-1)-\delta}{2}},
\end{array}\right.
$$

in addition, for $r>\frac{1}{3} R(t)$, further assume

$$
|Z \dot{\Phi}| \leq M \varepsilon R(t)^{1-3(\gamma-1)},\left|Z D_{t} \dot{\Phi}\right| \leq M \varepsilon R(t)^{-3(\gamma-1)},\left|\nabla_{x} Z \dot{\Phi}\right| \leq M \varepsilon R(t)^{-\frac{3(\gamma-1)}{2}} .
$$

Then for sufficiently small $\varepsilon>0$ and $0 \leq k \leq 2$, we have

$$
\begin{aligned}
& \int_{S_{T}}\left(R(T)^{\mu+2 k}\left(\nabla_{t, x}^{k} D_{t} \dot{\Phi}\right)^{2}+R(T)^{\mu-3(\gamma-1)+2 k}\left(\nabla_{t, x}^{k} \nabla_{x} \dot{\Phi}\right)^{2}\right) d S \\
& \quad+\int_{\Omega_{T}}\left(R(t)^{\mu-1-\delta+2 k}\left(\nabla_{t, x}^{k} D_{t} \dot{\Phi}\right)^{2}+R(t)^{\mu-1-3(\gamma-1)+2 k}\left(\nabla_{t, x}^{k} \nabla_{x} \dot{\Phi}\right)^{2}\right) d t d x \\
& \leq C \varepsilon^{2}
\end{aligned}
$$

and

$$
\begin{aligned}
& \int_{S_{T}}\left(R(T)^{\mu-\delta+6}\left(\nabla_{t, x}^{3} D_{t} \dot{\Phi}\right)^{2}+R(T)^{\mu-\delta-3(\gamma-1)+6}\left(\nabla_{t, x}^{3} \nabla_{x} \dot{\Phi}\right)^{2}\right) d S \\
& \quad+\int_{\Omega_{T}}\left(R(t)^{\mu+5-\delta}\left(\nabla_{t, x}^{3} D_{t} \dot{\Phi}\right)^{2}+R(t)^{\mu-\delta+5-3(\gamma-1)}\left(\nabla_{t, x}^{3} \nabla_{x} \dot{\Phi}\right)^{2}\right) d t d x \\
& \leq C \varepsilon^{2}
\end{aligned}
$$

where $\mu=6 \gamma-9,0<\delta \leq \frac{3(\gamma-1)}{5}, C>0$ is independent of $M$, and the domains $\Omega_{T}, S_{T}$ are defined in Section 4.

In order to prove Theorem 5.1, we will apply the induction on $k$ in (5.3)-(5.4) to establish the following estimates respectively:

(i) $D_{t} S^{k} \dot{\Phi}$ and $\nabla_{x} S^{k} \dot{\Phi}$ with $S=S_{0}^{l_{1}} Z^{l_{2}}\left(S_{0}=R(t) D_{t}\right)$ and $1 \leq k=l_{1}+l_{2} \leq 3$ (in this case, all the tangent derivatives of $\nabla_{x} \dot{\Phi}$ up to the third order are estimated, where the tangent derivative means the one of boundary $B_{T}$ );

(ii) $D_{t} S_{1} \dot{\Phi}$ and $\nabla_{x} S_{1} \nabla_{x} \dot{\Phi}$ with $S_{1}=r \partial_{r}$ (in this case, together with the case $k=1$ in (i), all the second order derivatives $\nabla_{t, x}^{2} \dot{\Phi}$ are analyzed);

(iii) $D_{t} S S_{1} \dot{\Phi}, \nabla_{x} S S_{1} \dot{\Phi}, D_{t} S_{1}^{2} \dot{\Phi}$ and $\nabla_{x} S_{1}^{3} \dot{\Phi}$ (in this case, together with the case $k=2$ in (i), all the estimates of third order derivatives $\nabla_{t, x}^{2} \dot{\Phi}$ are given); 
(iv) $D_{t} S^{2} S_{1} \dot{\Phi}, \nabla_{x} S^{2} S_{1} \dot{\Phi}, D_{t} S S_{1}^{2} \dot{\Phi}, \nabla_{x} S S_{1}^{2} \dot{\Phi}, D_{t} S_{1}^{3} \dot{\Phi}$ and $\nabla_{x} S_{1}^{3} \dot{\Phi}$ (in this case, together with the case $k=3$ in (i), all the fourth order derivatives $\nabla_{t, x}^{4} \dot{\Phi}$ are estimated).

These estimates will be given in Lemma 5.2-Lemma 5.5 respectively.

Firstly, we establish the tangent derivative estimates of $\dot{\Phi}$ under the suitable induction assumption. Set $S_{0}=R(t) D_{t}$ and $S^{m}=S_{0}^{l_{1}} Z^{l_{2}}$ with $m=l_{1}+l_{2}$, which are tangent to the boundary $\partial \Omega$. Then we have

Lemma 5.1 (Tangent derivative estimates) Under the assumptions of Theorem 5.1, if (5.3) holds for $0 \leq k \leq m$ with $1 \leq m \leq 2$, then

$$
\begin{aligned}
& R(T)^{\mu} \int_{S_{T}}\left(D_{t} S^{m} \dot{\Phi}\right)^{2} d S+R(T)^{\mu-3(\gamma-1)} \int_{S_{T}}\left(\nabla_{x} S^{m} \dot{\Phi}\right)^{2} d S \\
& \quad+\int_{\Omega_{T}}\left(R(t)^{\mu-1-\delta}\left(D_{t} S^{m} \dot{\Phi}\right)^{2}+R(t)^{\mu-1-3(\gamma-1)}\left(\nabla_{x} S^{m} \dot{\Phi}\right)^{2}\right) d t d x \\
& \leq C \varepsilon^{2}+C \varepsilon \int_{\Omega_{T}} \sum_{l=0}^{m}\left(R(t)^{\mu-1-\delta+2 l}\left(\nabla_{t, x}^{l} D_{t} \dot{\Phi}\right)^{2}+R(t)^{\mu-1-3(\gamma-1)+2 l}\left(\nabla_{t, x}^{l} \nabla_{x} \dot{\Phi}\right)^{2}\right) d t d x,
\end{aligned}
$$

where $0<\delta \leq \frac{3(\gamma-1)}{5}$

Especially, for $m=0$, the following estimate holds

$$
\begin{aligned}
& R(T)^{\mu} \int_{S_{T}}\left(D_{t} \dot{\Phi}\right)^{2} d S+R(T)^{\mu-3(\gamma-1)} \int_{S_{T}}\left(\nabla_{x} \dot{\Phi}\right)^{2} d S \\
& \quad+\int_{\Omega_{T}}\left(R(t)^{\mu-1-\delta}\left(D_{t} \dot{\Phi}\right)^{2}+R(t)^{\mu-1-3(\gamma-1)}\left(\nabla_{x} \dot{\Phi}\right)^{2}\right) d t d x \\
& \leq C \varepsilon^{2} .
\end{aligned}
$$

For $m=3$, if (5.2)-(5.3) hold, then

$$
\begin{aligned}
& R(T)^{\mu} \int_{S_{T}}\left(D_{t} S^{3} \dot{\Phi}\right)^{2} d S+R(T)^{\mu-3(\gamma-1)} \int_{S_{T}}\left(\nabla_{x} S^{3} \dot{\Phi}\right)^{2} d S \\
& \quad+\int_{\Omega_{T}}\left(R(t)^{\mu-1-\delta}\left(D_{t} S^{3} \dot{\Phi}\right)^{2}+R(t)^{\mu-1-3(\gamma-1)}\left(\nabla_{x} S^{3} \dot{\Phi}\right)^{2}\right) d t d x \\
& \leq C \varepsilon^{2}+C \varepsilon \int_{\Omega_{T}} \sum_{l=0}^{2}\left(R(t)^{\mu-1-\delta+2 l}\left(\nabla_{t, x}^{l} D_{t} \dot{\Phi}\right)^{2}+R(t)^{\mu-1-3(\gamma-1)+2 l}\left(\nabla_{t, x}^{l} \nabla_{x} \dot{\Phi}\right)^{2}\right) d t d x \\
& \quad+C \varepsilon \int_{\Omega_{T}}\left(R(t)^{\mu+5-\delta}\left(\nabla_{t, x}^{3} D_{t} \dot{\Phi}\right)^{2}+R(t)^{\mu+5-3(\gamma-1)-\delta}\left(\nabla_{t, x}^{3} \nabla_{x} \dot{\Phi}\right)^{2}\right) d t d x
\end{aligned}
$$

Remark 5.1 For the case when $m=0$ in (5.5), we do not require any induction assumption.

Remark 5.2 It is noted that the normal derivatives of $\dot{\Phi}$ are included on the right hand side of (5.5) and (5.6), which implies that we have not obtained the close estimates on the tangent derivative estimates of $\dot{\Phi}$. However, since the coefficients of normal derivatives of $\dot{\Phi}$ in (5.5) are small, then together with some subsequent normal derivative estimates, we can derive (5.3). 
Proof. Note that on $B_{T}$

$$
S^{m}\left(\sum_{i=1}^{3} x_{i} \partial_{i} \dot{\Phi}\right)=S^{m}\left(r \partial_{r} \dot{\Phi}\right)=0 .
$$

This, together with Theorem 4.1 and (3.5), yields

$$
\begin{aligned}
& R(T)^{\mu} \int_{S_{T}}\left(D_{t} S^{m} \dot{\Phi}\right)^{2} d S+R(T)^{\mu-3(\gamma-1)} \int_{S_{T}}\left(\nabla_{x} S^{m} \dot{\Phi}\right)^{2} d S \\
& \quad+\int_{\Omega_{T}}\left(R(t)^{\mu-1-\delta}\left(D_{t} S^{m} \dot{\Phi}\right)^{2}+R(t)^{\mu-1-3(\gamma-1)}\left(\nabla_{x} S^{m} \dot{\Phi}\right)^{2}\right) d t d x \\
& \leq C \int_{\Omega_{T}} \mathcal{L} S^{m} \dot{\Phi} \cdot \mathcal{M} S^{m} \dot{\Phi} d t d x+C \varepsilon^{2} .
\end{aligned}
$$

We divide the estimate (5.5) into three cases: (1) $S^{m}=S_{0}^{m}$, (2) $S^{m}=Z^{m}$, (3) $S^{m}=S_{0}^{l_{1}} Z^{l_{2}}(1 \leq$ $\left.l_{1}, l_{2} \leq m-1, l_{1}+l_{2}=m\right)$.

Case (1) $S^{m}=S_{0}^{m}$.

We now derive an explicit representation of $\mathcal{L} S_{0}^{m} \dot{\Phi}$ for later use. By direct computation, we have

$$
S_{0} \mathcal{L}=\mathcal{L} S_{0}-2 L \mathcal{L}+3(\gamma-1) \hat{c}^{2} \Delta
$$

By induction, for $1 \leq m \leq 3$, we obtain

$$
\mathcal{L} S_{0}^{m}=S_{0}^{m} \mathcal{L}+B_{1 m}+B_{2 m}
$$

with

$$
B_{1 m}=\sum_{0 \leq l \leq m-1} C_{l m} S_{0}^{l} \mathcal{L}+\sum_{0 \leq l \leq m-2} C_{l m}^{\prime} S_{0}^{l}\left(\hat{c}^{2} \Delta\right), B_{2 m}=-3 m(\gamma-1) S_{0}^{m-1}\left(\hat{c}^{2} \Delta\right),
$$

where $B_{2 m} \dot{\Phi}$ contains the $(m+1)$-th order (the highest order) derivatives of $\dot{\Phi}$, but $B_{1 m} \dot{\Phi}$ only includes the terms $\nabla_{t, x}^{\alpha} \dot{\Phi}$ with $|\alpha| \leq m$ (the lower order derivatives of $\dot{\Phi}$ ) and the term $\nabla_{t, x}^{m+1} \dot{\Phi}$ with small coefficients.

In addition, from equation (4.15), we have that for $0 \leq m \leq 3$

$$
S_{0}^{m} \mathcal{L} \dot{\Phi}=I_{1}^{m}+I_{2}^{m}+I_{3}^{m},
$$

where

$$
\begin{aligned}
I_{1}^{m} & =\sum_{i=1}^{3} \tilde{f}_{0 i} \partial_{i} D_{t} S_{0}^{m} \dot{\Phi}+\sum_{1 \leq i \neq j \leq 3} \tilde{f}_{i j} \partial_{i j}^{2} S_{0}^{m} \dot{\Phi}+\sum_{i=1}^{3} \tilde{f}_{i}^{2} \partial_{i}^{2} S_{0}^{m} \dot{\Phi} \\
I_{2}^{m} & =\sum_{i=1}^{3} \tilde{f}_{0 i}\left[S_{0}^{m}, \partial_{i} D_{t}\right] \dot{\Phi}+\sum_{0 \leq i \neq j \leq 3} \tilde{f}_{i j}\left[S_{0}^{m}, \partial_{i j}^{2}\right] \dot{\Phi}+\sum_{i=1}^{3} \tilde{f}_{i i}\left[S_{0}^{m}, \partial_{i}^{2}\right] \dot{\Phi} \\
I_{3}^{m} & =\sum_{1 \leq l \leq m} C_{l m}\left\{\sum_{l_{1}+l_{2}=l, l_{1} \geq 1} \tilde{C}_{l_{1} l_{2}}\left(\sum_{i=1}^{3}\left(S_{0}^{l_{1}} \tilde{f}_{0 i}\right) S_{0}^{l_{2}}\left(\partial_{i} D_{t} \dot{\Phi}\right)+\sum_{1 \leq i, j \leq 3}\left(S_{0}^{l_{1}} \tilde{f}_{i j}\right) S_{0}^{l_{2}}\left(\partial_{i j}^{2} \dot{\Phi}\right)\right)\right\}+S_{0}^{m} \tilde{f}_{0} .
\end{aligned}
$$


Based on the above preparation, we now treat $\int_{\Omega_{T}} \mathcal{L} S_{0}^{m} \dot{\Phi} \cdot \mathcal{M} S_{0}^{m} \dot{\Phi} d t d x$ on the right hand side of (5.8) as follows. This procedure is divided into five parts.

Part 1. Estimate on $\int_{\Omega_{T}} I_{1}^{m} \cdot \mathcal{M} S^{m} \dot{\Phi} d t d x$.

Note that for $C^{1}$-smooth functions $g$, one has

$$
D_{t} g=\partial_{t} g+\sum_{i=1}^{3} \partial_{i}\left(\frac{L}{R(t)} x_{i} g\right)-\frac{3 L}{R(t)} g .
$$

Then

$$
\int_{\Omega_{T}} D_{t} g d t d x=\int_{S_{T}} g d S-\int_{S^{0}} g d S-\int_{\Omega_{T}} \frac{3 L}{R(t)} g d t d x .
$$

In addition, for $m \leq 3$, direct computation yields

$$
\begin{aligned}
& I_{1}^{m} \cdot \mathcal{M} S_{0}^{m} \dot{\Phi} \\
& =D_{t}\left(-\frac{1}{2} a(t) R(t)^{\mu} \sum_{i, j=1}^{3}\left(\tilde{f}_{i j} \partial_{i} S_{0}^{m} \dot{\Phi} \partial_{j} S_{0}^{m} \dot{\Phi}\right)\right) \\
& \quad+\sum_{i=1}^{3} \partial_{i}\left(\frac{1}{2} a(t) R(t)^{\mu} \tilde{f}_{0 i}\left(D_{t} S_{0}^{m} \dot{\Phi}\right)^{2}+a(t) R(t)^{\mu} D_{t} S_{0}^{m} \dot{\Phi} \sum_{j=1}^{3} \tilde{f}_{i j} \partial_{j} S_{0}^{m} \dot{\Phi}\right) \\
& \quad-\frac{1}{2} a(t) R(t)^{\mu}\left(D_{t} S_{0}^{m} \dot{\Phi}\right)^{2} \sum_{i=1}^{3} \partial_{i} f_{0 i}-a(t) L R(t)^{\mu-1} \sum_{i, j=1}^{3}\left(\tilde{f}_{i j} \partial_{i} S_{0}^{m} \dot{\Phi} \partial_{j} S_{0}^{m} \dot{\Phi}\right) \\
& \quad-a(t) R(t)^{\mu} D_{t} S_{0}^{m} \dot{\Phi} \sum_{i, j=1}^{3}\left(\partial_{i} \tilde{f}_{i j} \partial_{j} S_{0}^{m} \dot{\Phi}\right)+\frac{1}{2} \sum_{i, j=1}^{3}\left(D_{t}\left(a(t) R(t)^{\mu} \tilde{f}_{i j}\right) \partial_{i} S_{0}^{m} \dot{\Phi} \partial_{j} S_{0}^{m} \dot{\Phi}\right) .
\end{aligned}
$$

On the other hand, by the expressions of $\tilde{f}_{i j}$ and $\tilde{f}_{0 i}$, and (5.7) on $B_{T}$, we observe that

$$
\begin{aligned}
& \sum_{i=1}^{3} x_{i} \cdot\left(\frac{1}{2} a(t) R(t)^{\mu} \tilde{f}_{0 i}\left(D_{t} S_{0}^{m} \dot{\Phi}\right)^{2}+a(t) R(t)^{\mu} D_{t} S_{0}^{m} \dot{\Phi} \sum_{j=1}^{3} \tilde{f}_{i j} \partial_{j} S_{0}^{m} \dot{\Phi}\right) \\
& =-a(t) R(t)^{\mu}\left(D_{t} S_{0}^{m} \dot{\Phi}\right)^{2} \sum_{i=1}^{3}\left(x_{i} \partial_{i} \dot{\Phi}\right)-a(t) R(t)^{\mu} D_{t} S_{0}^{m} \dot{\Phi} \sum_{i, j=1}^{3}\left(x_{i} \partial_{i} \dot{\Phi} \partial_{j} \dot{\Phi}\right) \\
& \quad-(\gamma-1) a(t) R(t)^{\mu} D_{t} S_{0}^{m} \dot{\Phi}\left(D_{t} \dot{\Phi}+\frac{1}{2} \sum_{k=1}^{3}\left(\partial_{k} \dot{\Phi}\right)^{2}\right) \sum_{i=1}^{3} x_{i} \partial_{i} S_{0}^{m} \dot{\Phi} \\
& =0
\end{aligned}
$$

Thus, by (5.14) together with (5.13) and (5.15), it follows from the expressions of $\tilde{f}_{i j}, \tilde{f}_{0 i}, \tilde{f}_{0}$ and assumption (5.1) that

$$
\left|\int_{\Omega_{T}} I_{1}^{m} \cdot \mathcal{M} S_{0}^{m} \dot{\Phi} d t d x\right|
$$




$$
\begin{aligned}
\leq & C \varepsilon^{2}+C \varepsilon\left(R(T)^{\mu} \int_{S_{T}}\left(D_{t} S_{0}^{m} \dot{\Phi}\right)^{2} d S+R(T)^{\mu-3(\gamma-1)} \int_{S_{T}}\left(\nabla_{x} S_{0}^{m} \dot{\Phi}\right)^{2} d S\right. \\
& +\int_{\Omega_{T}}\left(R(t)^{\mu-1-\delta}\left(D_{t} S_{0}^{m} \dot{\Phi}\right)^{2}+R(t)^{\mu-1-3(\gamma-1)}\left(\nabla_{x} S_{0}^{m} \dot{\Phi}\right)^{2} d t d x\right)
\end{aligned}
$$

where we have used

$$
\begin{aligned}
& \left|a(t) R(t)^{\mu}\left(D_{t} S_{0}^{m} \dot{\Phi}\right)^{2} \sum_{i=1}^{3} \partial_{i} \tilde{f}_{0 i}\right| \\
& \leq C \varepsilon R(t)^{\mu-1-\frac{3(\gamma-1)-\delta}{2}}\left(D_{t} S_{0}^{m} \dot{\Phi}\right)^{2} \\
& \leq C \varepsilon R(t)^{\mu-1-\delta}\left(D_{t} S_{0}^{m} \dot{\Phi}\right)^{2} \text { for } \quad 0<\delta \leq \gamma-1 .
\end{aligned}
$$

Part 2. Estimate on $\int_{\Omega_{T}} I_{2}^{m} \cdot \mathcal{M} S_{0}^{m} \dot{\Phi} d t d x$.

It follows from the expressions of $\tilde{f}_{i j}, \tilde{f}_{0 i}, \tilde{f}_{0}$ and (5.1) that

$$
\left|I_{2}^{m}\right| \leq C \varepsilon\left(R(t)^{-3(\gamma-1)-1+\frac{\delta}{2}} \sum_{0 \leq l \leq m-1}\left|\nabla_{x} S_{0}^{l+1} \dot{\Phi}\right|+R(t)^{-3(\gamma-1)} \sum_{0 \leq l \leq m-1}\left|\nabla_{x}^{2} S_{0}^{l} \dot{\Phi}\right|\right) .
$$

This derives that for $m \leq 2$

$\int_{\Omega_{T}}\left|I_{2}^{m} \cdot \mathcal{M} \dot{\Phi}\right| d t d x \leq C \varepsilon \int_{\Omega_{T}} \sum_{l=0}^{m}\left(R(t)^{\mu-1-\delta+2 l}\left(\nabla_{t, x}^{l} D_{t} \dot{\Phi}\right)^{2}+R(t)^{\mu-1-3(\gamma-1)+2 l}\left(\nabla_{t, x}^{l} \nabla_{x} \dot{\Phi}\right)^{2}\right) d t d x$,

and for $m=3$

$$
\begin{aligned}
& \int_{\Omega_{T}}\left|I_{2}^{m} \cdot \mathcal{M} \dot{\Phi}\right| d t d x \\
\leq & C \varepsilon \int_{\Omega_{T}} \sum_{l=0}^{2}\left(R(t)^{\mu-1-\delta+2 l}\left(\nabla_{t, x}^{l} D_{t} \dot{\Phi}\right)^{2}+R(t)^{\mu-1-3(\gamma-1)+2 l}\left(\nabla_{t, x}^{l} \nabla_{x} \dot{\Phi}\right)^{2}\right) d t d x \\
& +C \varepsilon \int_{\Omega_{T}}\left(R(t)^{\mu+5-\delta}\left(\nabla_{t, x}^{3} D_{t} \dot{\Phi}\right)^{2}+R(t)^{\mu+5-3(\gamma-1)-\delta}\left(\nabla_{t, x}^{3} \nabla_{x} \dot{\Phi}\right)^{2}\right) d t d x .
\end{aligned}
$$

Part 3. Estimate on $\int_{\Omega_{T}} I_{3}^{m} \cdot \mathcal{M} S_{0}^{m} \dot{\Phi} d t d x$.

First, we treat the case of $\int_{\Omega_{T}} I_{3}^{m} \cdot \mathcal{M} S_{0}^{m} \dot{\Phi} d x$ with $m \leq 2$.

For $m \leq 2$, as in Part 2, it follows from the expression of $f_{i}$ and assumption (5.1) that

$$
\left|I_{3}^{m}\right| \leq C \varepsilon\left(R(t)^{-3(\gamma-1)-1+\frac{\delta}{2}} \sum_{0 \leq l \leq m}\left|\nabla_{x} S_{0}^{l} \dot{\Phi}\right|+R(t)^{-3(\gamma-1)} \sum_{0 \leq l \leq m}\left|\nabla_{x}^{2} S_{0}^{l} \dot{\Phi}\right|\right),
$$

which yields for $m \leq 2$

$$
\int_{\Omega_{T}}\left|I_{3}^{m} \cdot \mathcal{M} S^{m} \dot{\Phi}\right| d x \leq C \varepsilon \sum_{l=0}^{m} \int_{\Omega_{T}}\left(R(t)^{\mu-1-\delta+2 l}\left(\nabla_{t, x}^{l} D_{t} \dot{\Phi}\right)^{2}+R(t)^{\mu-1-3(\gamma-1)+2 l}\left(\nabla_{t, x}^{l} \nabla_{x} \dot{\Phi}\right)^{2}\right) d t d x .
$$


Next we deal with $\int_{\Omega_{T}} I_{3}^{3} \cdot \mathcal{M} S_{0}^{3} \dot{\Phi} d t d x$. Note that the difficult terms in $I_{3}^{3}$ are the those that include the products of third order derivatives of $\dot{\Phi}$ because there are no related weighted $L^{\infty}$ estimates in (5.1). For the convenience, we decompose $I_{3}^{3}$ into $J_{1}$ and $J_{2}$ by using $S_{0}^{2}=L R(t) D_{t}+R(t)^{2} D_{t}^{2}$, where only $J_{2}$ contains the products of third order derivatives of $\dot{\Phi}$. Namely,

$$
I_{3}^{3}=J_{1}+J_{2}
$$

where

$$
\begin{aligned}
J_{1}= & \sum_{1 \leq l \leq 2} C_{l m}\left\{\sum_{l_{1}+l_{2}=l, l_{1} \geq 1} \tilde{C}_{l_{1} l_{2}}\left(\sum_{i=1}^{3}\left(S_{0}^{l_{1}} \tilde{f}_{0 i}\right) S_{0}^{l_{2}}\left(\partial_{i} D_{t} \dot{\Phi}\right)+\sum_{1 \leq i, j \leq 3}\left(S_{0}^{l_{1}} \tilde{f}_{i j}\right) S_{0}^{l_{2}}\left(\partial_{i j}^{2} \dot{\Phi}\right)\right)\right\} \\
& +C_{33} \sum_{\left(l_{1}, l_{2}\right) \neq(2,1)} \tilde{C}_{l_{1} l_{2}}\left(\sum_{i=1}^{3}\left(S_{0}^{l_{1}} \tilde{f}_{0 i}\right) S_{0}^{l_{2}}\left(\partial_{i} D_{t} \dot{\Phi}\right)+\sum_{1 \leq i, j \leq 3}\left(S_{0}^{l_{1}} \tilde{f}_{i j}\right) S_{0}^{l_{2}}\left(\partial_{i j}^{2} \dot{\Phi}\right)\right) \\
& +C_{33} \tilde{C}_{21}\left(\sum_{i=1}^{3}\left(L S_{0} \tilde{f}_{0 i}\right) S_{0}^{2}\left(\partial_{i} D_{t} \dot{\Phi}\right)+\sum_{1 \leq i, j \leq 3}\left(L S_{0} \tilde{f}_{i j}\right) S_{0}^{2}\left(\partial_{i j}^{2} \dot{\Phi}\right)\right)+S_{0}^{3} \tilde{f}_{0}
\end{aligned}
$$

and

$$
J_{2}=C_{33} \tilde{C}_{21}\left(\sum_{i=1}^{3}\left(R(t)^{2} D_{t}^{2} \tilde{f}_{0 i}\right) S_{0}\left(\partial_{i} D_{t} \dot{\Phi}\right)+\sum_{1 \leq i, j \leq 3}\left(R(t)^{2} D_{t}^{2} \tilde{f}_{i j}\right) S_{0}\left(\partial_{i j}^{2} \dot{\Phi}\right)\right) .
$$

By assumption (5.1) and the expressions of $\tilde{f}_{0 i}, \tilde{f}_{i j}, \tilde{f}_{0}$, direct computation yields

$$
\left|J_{1}\right| \leq C \varepsilon\left(R(t)^{-3(\gamma-1)-1+\frac{\delta}{2}} \sum_{0 \leq l \leq 3}\left|\nabla_{x} S_{0}^{l} \dot{\Phi}\right|+R(t)^{-3(\gamma-1)} \sum_{0 \leq l \leq 2}\left|\nabla_{x}^{2} S_{0}^{l} \dot{\Phi}\right|\right) .
$$

On the other hand, by the expression of $f_{i j}$, we further decompose $J_{2}$ into $J_{2}=J_{21}+J_{22}$ so that only $J_{22}$ contains the product terms of third order derivatives of $\dot{\Phi}$. More precisely,

$$
J_{2}=J_{21}+J_{22}
$$

with

$$
\begin{aligned}
J_{22}= & C_{33} \tilde{C}_{21}\left(-2 R(t)^{-1} \sum_{i=1}^{3}\left(\partial_{i} S_{0}^{2} \dot{\Phi}\right)^{2}-4 \sum_{i, j=1}^{3}\left(\partial_{i} \dot{\Phi} \partial_{j} S_{0}^{2} \dot{\Phi} \partial_{i j}^{2} S_{0} \dot{\Phi}\right)-(\gamma-1) D_{t} S_{0}^{2} \dot{\Phi} \sum_{i=1}^{3} \partial_{i}^{2} S_{0} \dot{\Phi}\right. \\
& \left.-(\gamma-1) \sum_{i, j=1}^{3}\left(\partial_{i} \dot{\Phi} \partial_{i} S_{0}^{2} \dot{\Phi} \partial_{j}^{2} S_{0} \dot{\Phi}\right)\right),
\end{aligned}
$$

and

$$
\left|J_{21}\right| \leq C \varepsilon\left(R(t)^{-3(\gamma-1)-1+\frac{\delta}{2}} \sum_{0 \leq l \leq 3}\left|\nabla_{x} S_{0}^{l} \dot{\Phi}\right|+R(t)^{-3(\gamma-1)} \sum_{0 \leq l \leq 2}\left|\nabla_{x}^{2} S_{0}^{l} \dot{\Phi}\right|\right) .
$$

Combining (5.22) and (5.23) yields

$$
\int_{\Omega_{T}}\left|\left(J_{1}+J_{21}\right) \cdot \mathcal{M} S_{0}^{3} \dot{\Phi}\right| d t d x
$$




$$
\begin{aligned}
\leq & C \varepsilon \sum_{l=0}^{2} \int_{\Omega_{T}}\left(R(t)^{\mu-1-\delta+2 l}\left(\nabla_{t, x}^{l} D_{t} \dot{\Phi}\right)^{2}+R(t)^{\mu-1-3(\gamma-1)+2 l}\left(\nabla_{t, x}^{l} \nabla_{x} \dot{\Phi}\right)^{2}\right) d t d x \\
& +\int_{\Omega_{T}}\left(R(t)^{\mu+5-\delta}\left(\nabla_{t, x}^{l} D_{t} \dot{\Phi}\right)^{2}+R(t)^{\mu+5-3(\gamma-1)-\delta}\left(\nabla_{t, x}^{l} \nabla_{x} \dot{\Phi}\right)^{2}\right) d t d x .
\end{aligned}
$$

Finally, we estimate $\int_{\Omega_{T}}\left|J_{22} \cdot \mathcal{M} S_{0}^{3} \dot{\Phi}\right| d t d x$. To overcome the difficulty induced by the lack of weighted $L^{\infty}$ estimates of $\left|\nabla_{t, x}^{3} \dot{\Phi}\right|$ in $J_{22}$, we will use the interpolation inequalities in Corollary 2.1 and Lemma 2.4. In fact, by (5.1) and the expression of $J_{22}$, it is sufficient to estimate some typical terms in $\int_{D_{T}}\left|J_{22} \cdot \mathcal{M} S_{0}^{3} \dot{\Phi}\right| d t d x$ as follows:

(A) Estimate on $\left|R(t)^{\mu-1}\left(\partial_{i} S_{0}^{2} \dot{\Phi}\right)^{2} D_{t} S_{0}^{3} \dot{\Phi}\right|_{L^{1}\left(\Omega_{T}\right)}$.

$$
\begin{aligned}
& \left|R(t)^{\mu-1}\left(\partial_{i} S_{0}^{2} \dot{\Phi}\right)^{2} D_{t} S_{0}^{3} \dot{\Phi}\right|_{L^{1}\left(\Omega_{T}\right)} \\
& \leq\left|R(t)^{\frac{2 \mu+2-\delta}{4}} \partial_{i} S_{0}^{2} \dot{\Phi}\right|_{L^{4}}^{2}\left|R(t)^{\frac{\mu-1-\delta}{2}} D_{t} S_{0}^{3} \dot{\Phi}\right|_{L^{2}} \\
& \leq C \varepsilon\left(\sum_{k=0}^{2}\left|R(t)^{\frac{\mu+3-\delta}{2}-k} \nabla_{t, x}^{3-k} S_{0} \dot{\Phi}\right|_{L^{2}}^{\frac{1}{2}}\right)^{2}\left|R(t)^{\frac{\mu-1-\delta}{2}} D_{t} S_{0}^{3} \dot{\Phi}\right|_{L^{2}} \quad \text { (Applying Lemma 2.4 (i) for } \dot{\Phi} \text { ) } \\
& \leq C \varepsilon \int_{\Omega_{T}} \sum_{l=0}^{2}\left(R(t)^{\mu-1-\delta+2 l}\left(\nabla_{t, x}^{l} D_{t} \dot{\Phi}\right)^{2}+R(t)^{\mu-1-3(\gamma-1)+2 l}\left(\nabla_{t, x}^{l} \nabla_{x} \dot{\Phi}\right)^{2}\right) d t d x \\
& \quad+C \varepsilon \int_{\Omega_{T}}\left(R(t)^{\mu+5-\delta}\left(\nabla_{t, x}^{3} D_{t} \dot{\Phi}\right)^{2}+R(t)^{\mu+5-3(\gamma-1)-\delta}\left(\nabla_{t, x}^{3} \nabla_{x} \dot{\Phi}\right)^{2}\right) d t d x .
\end{aligned}
$$

(B) Estimate on $\left|R(t)^{\mu} \partial_{i} \dot{\Phi} \partial_{j} S_{0}^{2} \dot{\Phi} \partial_{i j} S_{0} \dot{\Phi} D_{t} S_{0}^{3} \dot{\Phi}\right|_{L^{1}\left(\Omega_{T}\right)}$ •

$$
\begin{aligned}
& \left|R(t)^{\mu} \partial_{i} \dot{\Phi} \partial_{j} S_{0}^{2} \dot{\Phi} \partial_{i j}^{2} S_{0} \dot{\Phi} D_{t} S_{0}^{3} \dot{\Phi}\right|_{L^{1}\left(\Omega_{T}\right)} \\
& \leq C \varepsilon\left|R(t)^{\mu-3(\gamma-1)+\frac{\delta}{2}} \partial_{j} S_{0}^{2} \dot{\Phi} \partial_{i j}^{2} S_{0} \dot{\Phi} D_{t} S_{0}^{3} \dot{\Phi}\right|_{L^{1}\left(\Omega_{T}\right)} \quad \text { (By assumption (5.1)) } \\
& \leq C \varepsilon\left|R(t)^{\frac{2 \mu+2-\delta}{4}} \partial_{j} S_{0}^{2} \dot{\Phi}\right|_{L^{4}}\left|R(t)^{\frac{\mu+3-\delta}{2}} \partial_{i j}^{2} S_{0} \dot{\Phi}\right|_{L^{4}}\left|R(t)^{\frac{\mu-1-\delta}{2}} D_{t} S_{0}^{3} \dot{\Phi}\right|_{L^{2}} \\
& \leq C \varepsilon\left(\sum_{k=0}^{2}\left|R(t)^{\frac{\mu+3-\delta}{2}-k} \nabla_{t, x}^{3-k} S_{0} \dot{\Phi}\right|_{L^{2}}^{\frac{1}{2}}\right)\left(\sum_{k=0}^{2}\left|R(t)^{\frac{\mu+3-\delta}{2}-k} \nabla_{t, x}^{3-k} S_{0} \dot{\Phi}\right|_{L^{2}}^{\frac{1}{2}}\right)\left|R(t)^{\frac{\mu-1-\delta}{2}} D_{t} S_{0}^{3} \dot{\Phi}\right|_{L^{2}} \\
& \text { (Applying Lemma } 2.4 \text { (i)-(ii) for } \dot{\Phi} \text { ) } \\
& \leq C \varepsilon \int_{\Omega_{T}} \sum_{l=0}^{2}\left(R(t)^{\mu-1-\delta+2 l}\left(\nabla_{t, x}^{l} D_{t} \dot{\Phi}\right)^{2}+R(t)^{\mu-1-3(\gamma-1)+2 l}\left(\nabla_{t, x}^{l} \nabla_{x} \dot{\Phi}\right)^{2}\right) d t d x \\
& +C \varepsilon \int_{\Omega_{T}}\left(R(t)^{\mu+5-\delta}\left(\nabla_{t, x}^{3} D_{t} \dot{\Phi}\right)^{2}+R(t)^{\mu+5-3(\gamma-1)-\delta}\left(\nabla_{t, x}^{3} \nabla_{x} \dot{\Phi}\right)^{2}\right) d t d x .
\end{aligned}
$$

(C) Estimate on $\left|R(t)^{\mu} D_{t} S_{0}^{2} \dot{\Phi} \partial_{i}^{2} S_{0} \dot{\Phi} D_{t} S_{0}^{3} \dot{\Phi}\right|_{L^{1}\left(\Omega_{T}\right)}$.

$$
\left|R(t)^{\mu} D_{t} S_{0}^{2} \dot{\Phi} \partial_{i}^{2} S_{0} \dot{\Phi} D_{t} S_{0}^{3} \dot{\Phi}\right|_{L^{1}\left(\Omega_{T}\right)}
$$




$$
\begin{aligned}
& \leq C\left|R(t)^{\frac{2 \mu+2-\delta}{4}} D_{t} S_{0}^{2} \dot{\Phi}\right|_{L^{4}}\left|R(t)^{\frac{\mu+3-\delta}{2}} \partial_{i}^{2} S_{0} \dot{\Phi}\right|_{L^{4}}\left|R(t)^{\frac{\mu-1-\delta}{2}} D_{t} S_{0}^{3} \dot{\Phi}\right|_{L^{2}} \\
& \leq C \varepsilon\left(\sum_{k=0}^{2}\left|R(t)^{\frac{\mu+3-\delta}{2}-k} \nabla_{t, x}^{3-k} S_{0} \dot{\Phi}\right|_{L^{2}}^{\frac{1}{2}}\right)\left(\sum_{k=0}^{2}\left|R(t)^{\frac{\mu+3-\delta}{2}-k} \nabla_{t, x}^{3-k} S_{0} \dot{\Phi}\right|_{L^{2}}^{\frac{1}{2}}\right)\left|R(t)^{\frac{\mu-1-\delta}{2}} D_{t} S_{0}^{3} \dot{\Phi}\right|_{L^{2}}
\end{aligned}
$$

(Applying Lemma 2.4 (i)-(ii) for $\dot{\Phi}$ )

$$
\begin{aligned}
\leq & C \varepsilon \int_{\Omega_{T}} \sum_{l=0}^{2}\left(R(t)^{\mu-1-\delta+2 l}\left(\nabla_{t, x}^{l} D_{t} \dot{\Phi}\right)^{2}+R(t)^{\mu-1-3(\gamma-1)+2 l}\left(\nabla_{t, x}^{l} \nabla_{x} \dot{\Phi}\right)^{2}\right) d t d x \\
& +C \varepsilon \int_{\Omega_{T}}\left(R(t)^{\mu+5-\delta}\left(\nabla_{t, x}^{3} D_{t} \dot{\Phi}\right)^{2}+R(t)^{\mu+5-3(\gamma-1)-\delta}\left(\nabla_{t, x}^{3} \nabla_{x} \dot{\Phi}\right)^{2}\right) d t d x .
\end{aligned}
$$

Combining (5.25)-(5.27) gives

$$
\begin{aligned}
& \int_{\Omega_{T}}\left|J_{22} \cdot \mathcal{M} S_{0}^{3} \dot{\Phi}\right| d t d x \\
& \leq C \varepsilon \int_{\Omega_{T}} \sum_{l=0}^{2}\left(R(t)^{\mu-1-\delta+2 l}\left(\nabla_{t, x}^{l} D_{t} \dot{\Phi}\right)^{2}+R(t)^{\mu-1-3(\gamma-1)+2 l}\left(\nabla_{t, x}^{l} \nabla_{x} \dot{\Phi}\right)^{2}\right) d t d x \\
& \quad+C \varepsilon \int_{\Omega_{T}}\left(R(t)^{\mu+5-\delta}\left(\nabla_{t, x}^{3} D_{t} \dot{\Phi}\right)^{2}+R(t)^{\mu+5-3(\gamma-1)-\delta}\left(\nabla_{t, x}^{3} \nabla_{x} \dot{\Phi}\right)^{2}\right) d t d x .
\end{aligned}
$$

This, together with (5.19) and (5.24), yields for $m \leq 2$

$$
\int_{\Omega_{T}}\left|I_{3}^{m} \cdot \mathcal{M} S_{0}^{m} \dot{\Phi}\right| d t d x \leq C \varepsilon \sum_{l=0}^{m}\left(\left|R(t)^{\frac{\mu-1-\delta+2 l}{2}} \nabla_{t, x}^{l} D_{t} \dot{\Phi}\right|_{L^{2}}^{2}+\left|R(t)^{\frac{\mu-1-3(\gamma-1)+2 l}{2}} \nabla_{t, x}^{l} \nabla_{x} \dot{\Phi}\right|_{L^{2}}^{2}\right),
$$

and for $m=3$

$$
\begin{aligned}
& \int_{\Omega_{T}}\left|I_{3}^{m} \cdot \mathcal{M} S_{0}^{3} \dot{\Phi}\right| d t d x \\
\leq & C \varepsilon \int_{\Omega_{T}} \sum_{l=0}^{2}\left(R(t)^{\mu-1-\delta+2 l}\left(\nabla_{t, x}^{l} D_{t} \dot{\Phi}\right)^{2}+R(t)^{\mu-1-3(\gamma-1)+2 l}\left(\nabla_{t, x}^{l} \nabla_{x} \dot{\Phi}\right)^{2}\right) d t d x \\
& +C \varepsilon \int_{\Omega_{T}}\left(R(t)^{\mu+5-\delta}\left(\nabla_{t, x}^{3} D_{t} \dot{\Phi}\right)^{2}+R(t)^{\mu+5-3(\gamma-1)-\delta}\left(\nabla_{t, x}^{3} \nabla_{x} \dot{\Phi}\right)^{2}\right) d t d x .
\end{aligned}
$$

Part 4. Estimate on $\int_{\Omega_{T}} B_{1 m} \dot{\Phi} \cdot \mathcal{M} S_{0}^{m} \dot{\Phi} d t d x$.

First, from the expressions of $B_{1 m}$ and $\dot{f}$, and by (5.1), we have

$$
\begin{aligned}
\left|B_{11} \dot{\Phi}\right| \leq & C \varepsilon\left(R(t)^{-3(\gamma-1)+\frac{\delta}{2}}\left|\nabla_{x} D_{t} \dot{\Phi}\right|+R(t)^{-3(\gamma-1)}\left|\nabla_{x}^{2} \dot{\Phi}\right|+R(t)^{-3(\gamma-1)-1+\frac{\delta}{2}}\left|\nabla_{x} \dot{\Phi}\right|\right) \\
\left|B_{1 m} \dot{\Phi}\right| \leq & C \varepsilon \sum_{0 \leq l \leq m-1}\left(R(t)^{-3(\gamma-1)+\frac{\delta}{2}}\left|\nabla_{x} S_{0}^{l} D_{t} \dot{\Phi}\right|+R(t)^{-3(\gamma-1)}\left|\nabla_{x}^{2} S_{0}^{l} \dot{\Phi}\right|+R(t)^{-3(\gamma-1)-1+\frac{\delta}{2}}\left|\nabla_{x} S_{0}^{l} \dot{\Phi}\right|\right. \\
& \left.+R(t)^{-\frac{3(\gamma-1)-\delta}{2}-1}\left|D_{t} S_{0}^{l} \dot{\Phi}\right|\right)+C R(t)^{-3(\gamma-1)} \sum_{0 \leq l \leq m-2}\left|\Delta S_{0}^{l} \dot{\Phi}\right|, \quad m=2,3
\end{aligned}
$$


Since (5.3) holds for $l \leq m-1$, we have from (5.29)-(5.30) that, for $m \leq 2$,

$$
\begin{aligned}
& \int_{\Omega_{T}}\left|B_{1 m} \dot{\Phi} \cdot \mathcal{M} S_{0}^{m} \dot{\Phi}\right| d t d x \\
& \leq C \varepsilon \sum_{l=0}^{m} \int_{\Omega_{T}}\left(R(t)^{\mu-1-\delta+2 l}\left(\nabla_{t, x}^{l} D_{t} \dot{\Phi}\right)^{2}+R(t)^{\mu-1-3(\gamma-1)+2 l}\left(\nabla_{t, x}^{l} \nabla_{x} \dot{\Phi}\right)^{2}\right) d t d x \\
& \quad+C \varepsilon\left(\int_{\Omega_{T}} R(t)^{\mu-1-\delta}\left(D_{t} S_{0}^{m} \dot{\Phi}\right)^{2} d t d x\right)^{\frac{1}{2}},
\end{aligned}
$$

and for $m=3$,

$$
\begin{aligned}
& \int_{\Omega_{T}}\left|B_{1 m} \dot{\Phi} \cdot \mathcal{M} S_{0}^{m} \dot{\Phi}\right| d t d x \\
& \leq C \varepsilon \int_{\Omega_{T}} \sum_{l=0}^{2}\left(R(t)^{\mu-1-\delta+2 l}\left(\nabla_{t, x}^{l} D_{t} \dot{\Phi}\right)^{2}+R(t)^{\mu-1-3(\gamma-1)+2 l}\left(\nabla_{t, x}^{l} \nabla_{x} \dot{\Phi}\right)^{2}\right) d t d x \\
& \quad+C \varepsilon \int_{\Omega_{T}}\left(R(t)^{\mu+5-\delta}\left(\nabla_{t, x}^{3} D_{t} \dot{\Phi}\right)^{2}+R(t)^{\mu+5-3(\gamma-1)-\delta}\left(\nabla_{t, x}^{3} \nabla_{x} \dot{\Phi}\right)^{2}\right) d t d x \\
& \quad+C \varepsilon\left(\int_{\Omega_{T}} R(t)^{\mu-1-\delta}\left(D_{t} S_{0}^{3} \dot{\Phi}\right)^{2} d t d x\right)^{\frac{1}{2}} .
\end{aligned}
$$

Part 5. Estimate on $\int_{\Omega_{T}} B_{2 m} \dot{\Phi} \cdot \mathcal{M} S_{0}^{m} \dot{\Phi} d t d x$.

Note that $B_{2 m} \dot{\Phi}$ contains the $(m+1)$-th (the highest order) derivatives of $\dot{\Phi}$ and then $B_{2 m} \dot{\Phi} \cdot \mathcal{M} S^{m} \Phi$ contains the term $\nabla_{t, x}^{\alpha} \dot{\Phi} \nabla_{t, x}^{\beta} \dot{\Phi}(|\alpha|=|\beta|=m+1)$. Thanks to the definite sign of the coefficient $-3 m(\gamma-1)<0$ in the $B_{2 m}$ and the (3.1), $\nabla_{t, x}^{\alpha} \dot{\Phi} \nabla_{t, x}^{\beta} \dot{\Phi}$ with $|\alpha|=|\beta|=m+1$ can be controlled as follows.

Since

$$
D_{t} S_{0}^{m} \dot{\Phi}=\sum_{0 \leq l \leq m-1} C_{l m} D_{t} S_{0}^{l} \dot{\Phi}+R(t) S_{0}^{m-1} D_{t}^{2} \dot{\Phi}
$$

it follows from (3.1) that

$$
D_{t} S_{0}^{m} \dot{\Phi}=\sum_{0 \leq l \leq m-1} C_{l m} D_{t} S_{0}^{l} \dot{\Phi}+R(t) S_{0}^{m-1}\left(\hat{c}^{2} \Delta \dot{\Phi}-\frac{3 L(\gamma-1)}{R(t)} D_{t} \dot{\Phi}+\mathcal{L} \dot{\Phi}\right) .
$$

Direct computation yields

$$
\begin{aligned}
& \int_{\Omega_{T}} B_{2 m} \dot{\Phi} \cdot \mathcal{M} S_{0}^{m} \dot{\Phi} d t d x \\
& =\int_{\Omega_{T}}-3 m(\gamma-1) R(t)^{\mu} a(t)\left\{S_{0}^{m-1}\left(\hat{c}^{2} \Delta \dot{\Phi}\right)^{2}\right. \\
& \left.\quad+\left(\sum_{0 \leq l \leq m-1} C_{l m} D_{t} S_{0}^{l} \dot{\Phi}+R(t) S_{0}^{m-1}\left(\mathcal{L} \dot{\Phi}-\frac{3 L(\gamma-1)}{R(t)} D_{t} \dot{\Phi}\right)\right) S_{0}^{m-1}\left(\hat{c}^{2} \Delta \dot{\Phi}\right)\right\} d t d x
\end{aligned}
$$




$$
\begin{aligned}
\leq-3 m(\gamma-1) & \int_{\Omega_{T}} R(t)^{\mu} a(t)\left(\sum_{0 \leq l \leq m-1} C_{l m} D_{t} S_{0}^{l} \dot{\Phi}\right. \\
& \left.+R(t) S_{0}^{m-1}\left(\mathcal{L} \dot{\Phi}-\frac{3 L(\gamma-1)}{R(t)} D_{t} \dot{\Phi}\right)\right) S_{0}^{m-1}\left(\hat{c}^{2} \Delta \dot{\Phi}\right) d t d x
\end{aligned}
$$

Note that $\sum_{0 \leq l \leq m-1} C_{l m} D_{t} S_{0}^{l} \dot{\Phi}-R(t) S_{0}^{m-1}\left(\frac{3 L(\gamma-1)}{R(t)} D_{t} \dot{\Phi}\right)$ only contains at most $m$-th order derivatives of $\dot{\Phi}$, then we have by (5.1) that, for $l \leq m-1$,

$$
\int_{\Omega_{T}} R(t)^{\mu-1-\delta}\left(\sum_{0 \leq l \leq m-1} C_{l m} D_{t} S_{0}^{l} \dot{\Phi}-R(t) S_{0}^{m-1}\left(\frac{3 L(\gamma-1)}{R(t)} D_{t} \dot{\Phi}\right)\right)^{2} d t d x \leq C \varepsilon^{2} .
$$

On the other hand, we have

$$
\left|S_{0}^{m-1} \mathcal{L} \dot{\Phi}\right| \leq C \varepsilon\left(\sum_{0 \leq l \leq m} R(t)^{-3(\gamma-1)-1+\frac{\delta}{2}}\left|\nabla_{x} S_{0}^{l} \dot{\Phi}\right|+\sum_{0 \leq l \leq m-1} R(t)^{-3(\gamma-1)}\left|\nabla_{x}^{2} S_{0}^{l} \dot{\Phi}\right|\right) .
$$

Therefore, putting (5.37)-(5.38) into (5.36) yields for $m \leq 2$

$$
\begin{aligned}
& \int_{\Omega_{T}}\left|B_{2 m} \dot{\Phi} \cdot \mathcal{M} S_{0}^{m} \dot{\Phi}\right| d t d x \\
& \leq C \varepsilon^{2}+C \varepsilon \sum_{l=0}^{m} \int_{\Omega_{T}}\left(R(t)^{\mu-1-\delta+2 l}\left(\nabla_{t, x}^{l} D_{t} \dot{\Phi}\right)^{2}+R(t)^{\mu-1-3(\gamma-1)+2 l}\left(\nabla_{t, x}^{l} \nabla_{x} \dot{\Phi}\right)^{2}\right) d t d x
\end{aligned}
$$

and for $m=3$

$$
\begin{aligned}
& \int_{\Omega_{T}}\left|B_{2 m} \dot{\Phi} \cdot \mathcal{M} S_{0}^{m} \dot{\Phi}\right| d t d x \\
\leq & C \varepsilon^{2}+C \varepsilon \int_{\Omega_{T}} \sum_{l=0}^{2}\left(R(t)^{\mu-1-\delta+2 l}\left(\nabla_{t, x}^{l} D_{t} \dot{\Phi}\right)^{2}+R(t)^{\mu-1-3(\gamma-1)+2 l}\left(\nabla_{t, x}^{l} \nabla_{x} \dot{\Phi}\right)^{2}\right) d t d x \\
& +C \varepsilon \int_{\Omega_{T}}\left(R(t)^{\mu+5-\delta}\left(\nabla_{t, x}^{3} D_{t} \dot{\Phi}\right)^{2}+R(t)^{\mu+5-3(\gamma-1)-\delta}\left(\nabla_{t, x}^{3} \nabla_{x} \dot{\Phi}\right)^{2}\right) d t d x
\end{aligned}
$$

Consequently, putting (5.17)-(5.18), (5.29), (5.32), (5.37)-(5.38) into (5.8), we complete the proofs of (5.4) and (5.6) for $S^{m}=S_{0}^{m}$.

For the case of $m=0,(5.5)$ comes directly from Theorem 4.1, (5.17)-(5.18) and (5.29).

Case (2) $\quad S^{m}=Z^{m}$.

By direct computation, we have

$$
Z \mathcal{L}=\mathcal{L} Z
$$

that implies

$$
\mathcal{L} Z^{m}=Z^{m} \mathcal{L} .
$$

In addition, from equation (4.15), we have that for $0 \leq m \leq 3$,

$$
Z^{m} \mathcal{L} \dot{\Phi}=K_{1}^{m}+K_{2}^{m}+K_{3}^{m},
$$


where

$$
\begin{aligned}
K_{1}^{m} & =\sum_{i=1}^{3} \tilde{f}_{0 i} \partial_{i} D_{t} Z^{m} \dot{\Phi}+\sum_{1 \leq i \neq j \leq 3} \tilde{f}_{i j}^{2} \partial_{i j} Z^{m} \dot{\Phi}+\sum_{i=1}^{3} \tilde{f}_{i i} \partial_{i}^{2} Z^{m} \dot{\Phi}, \\
K_{2}^{m} & =\sum_{i=1}^{3} \tilde{f}_{0 i}\left[Z^{m}, \partial_{i} D_{t}\right] \dot{\Phi}+\sum_{0 \leq i \neq j \leq 3} \tilde{f}_{i j}\left[Z^{m}, \partial_{i j}^{2}\right] \dot{\Phi}+\sum_{i=1}^{3} \tilde{f}_{i i}\left[Z^{m}, \partial_{i}^{2}\right] \dot{\Phi}, \\
K_{3}^{m} & =\sum_{1 \leq l \leq m} C_{l m}\left\{\sum_{l_{1}+l_{2}=l, l_{1} \geq 1} \tilde{C}_{l_{1} l_{2}}\left(\sum_{i=1}^{3}\left(Z^{l_{1}} \tilde{f}_{0 i}\right) Z^{l_{2}}\left(\partial_{i} D_{t} \dot{\Phi}\right)+\sum_{1 \leq i, j \leq 3}\left(Z^{l_{1}} \tilde{f}_{i j}\right) Z^{l_{2}}\left(\partial_{i j}^{2} \dot{\Phi}\right)\right)\right\}+Z^{m} \tilde{f}_{0} .
\end{aligned}
$$

Based on the above preparation, we now estimate $\int_{\Omega_{T}} \mathcal{L} Z^{m} \dot{\Phi} \cdot \mathcal{M} Z^{m} \dot{\Phi} d t d x$ on the right hand side of (5.8) in the following three steps.

Part 1. Estimate on $\int_{\Omega_{T}} K_{1}^{m} \cdot \mathcal{M} S^{m} \dot{\Phi} d t d x$.

Replacing $S_{0}$ in (5.16) by $Z$, we have as in (5.16),

$$
\begin{aligned}
& \left|\int_{\Omega_{T}} K_{1}^{m} \cdot \mathcal{M} Z^{m} \dot{\Phi} d t d x\right| \\
& \leq C \varepsilon^{2}+C \varepsilon\left(R(T)^{\mu} \int_{S_{T}}\left(D_{t} Z^{m} \dot{\Phi}\right)^{2} d S+R(T)^{\mu-3(\gamma-1)} \int_{S_{T}}\left(\nabla_{x} Z^{m} \dot{\Phi}\right)^{2} d S\right. \\
& \quad+\int_{\Omega_{T}}\left(R(t)^{\mu-1-\delta}\left(D_{t} Z^{m} \dot{\Phi}\right)^{2}+R(t)^{\mu-1-3(\gamma-1)}\left(D_{t} Z^{m} \dot{\Phi}\right)^{2} d t d x\right) .
\end{aligned}
$$

Part 2. Estimate on $\int_{\Omega_{T}} K_{2}^{m} \cdot \mathcal{M} Z^{m} \dot{\Phi} d t d x$.

Direct computation yields

$$
\left|K_{2}^{m}\right| \leq C\left(\sum_{i=1}^{3}\left|\tilde{f}_{0 i}\right| \sum_{0 \leq l \leq m-1}\left|\nabla_{x} Z^{l} D_{t} \dot{\Phi}\right|+\sum_{i, j=1}^{3}\left|\tilde{f}_{i j}\right| \sum_{0 \leq l \leq m-2}\left|\nabla_{x}^{2} Z^{l} \dot{\Phi}\right|\right) .
$$

Then it follows from the expressions of $\tilde{f}_{i j}, \tilde{f}_{0 i}$ and (5.1) that

$$
\left|K_{2}^{m}\right| \leq C \varepsilon\left(R(t)^{-3(\gamma-1)+\frac{\delta}{2}} \sum_{0 \leq l \leq m-1}\left|\nabla_{x} Z^{l} D_{t} \dot{\Phi}\right|+R(t)^{-3(\gamma-1)} \sum_{0 \leq l \leq m-2}\left|\nabla_{x}^{2} Z^{l} \dot{\Phi}\right|\right)
$$

which gives that for $m \leq 2$,

$$
\begin{aligned}
& \int_{\Omega_{T}}\left|K_{2}^{m} \cdot \mathcal{M} Z^{m} \dot{\Phi}\right| d t d x \\
& \leq C \varepsilon \sum_{l=0}^{m} \int_{\Omega_{T}}\left(R(t)^{\mu-1-\delta+2 l}\left(\nabla_{t, x}^{l} D_{t} \dot{\Phi}\right)^{2}+R(t)^{\mu-1-3(\gamma-1)+2 l}\left(\nabla_{t, x}^{l} \nabla_{x} \dot{\Phi}\right)^{2}\right) d t d x
\end{aligned}
$$

and for $m=3$,

$$
\int_{\Omega_{T}}\left|K_{2}^{m} \cdot \mathcal{M} Z^{m} \dot{\Phi}\right| d t d x
$$




$$
\begin{aligned}
\leq & C \varepsilon \int_{\Omega_{T}} \sum_{l=0}^{2}\left(R(t)^{\mu-1-\delta+2 l}\left(\nabla_{t, x}^{l} D_{t} \dot{\Phi}\right)^{2}+R(t)^{\mu-1-3(\gamma-1)+2 l}\left(\nabla_{t, x}^{l} \nabla_{x} \dot{\Phi}\right)^{2}\right) d t d x \\
& +C \varepsilon \int_{\Omega_{T}}\left(R(t)^{\mu+5-\delta}\left(\nabla_{t, x}^{3} D_{t} \dot{\Phi}\right)^{2}+R(t)^{\mu+5-3(\gamma-1)-\delta}\left(\nabla_{t, x}^{3} \nabla_{x} \dot{\Phi}\right)^{2}\right) d t d x .
\end{aligned}
$$

Part 3. Estimate on $\int_{\Omega_{T}} K_{3}^{m} \cdot \mathcal{M} Z^{m} \dot{\Phi} d t d x$.

For $m=1$, it follows from the expressions of $\tilde{f}_{i j}, \tilde{f}_{0 i}, \tilde{f}_{0}$ and (5.1) that

$$
\left|K_{3}^{1}\right| \leq C \varepsilon\left(R(t)^{-3(\gamma-1)-1+\frac{\delta}{2}}\left(\left|\nabla_{x} \dot{\Phi}\right|+\left|\nabla_{x} Z \dot{\Phi}\right|\right)+R(t)^{-3(\gamma-1)}\left|\nabla_{x}^{2} \dot{\Phi}\right|\right) .
$$

This yields

$$
\int_{\Omega_{T}}\left|K_{3}^{1} \cdot \mathcal{M} \dot{\Phi}\right| d t d x \leq C \varepsilon \sum_{l=0}^{1} \int_{\Omega_{T}}\left(R(t)^{\mu-1-\delta+2 l}\left(\nabla_{t, x}^{l} D_{t} \dot{\Phi}\right)^{2}+R(t)^{\mu-1-3(\gamma-1)+2 l}\left(\nabla_{t, x}^{l} \nabla_{x} \dot{\Phi}\right)^{2}\right) d t d x .
$$

For $m=2$,

$$
K_{3}^{2}=K_{31}^{2}+K_{32}^{2}
$$

where

$$
\begin{aligned}
K_{31}^{2}= & \sum_{1 \leq l \leq 2} C_{l 2}\left\{\sum_{l_{1}+l_{2}=l, l_{1} \geq 1} \tilde{C}_{l_{1} l_{2}}\left(\sum_{i=1}^{3}\left(Z^{l_{1}} \tilde{f}_{0 i}\right) Z^{l_{2}}\left(\partial_{i} D_{t} \dot{\Phi}\right)+\sum_{1 \leq i, j \leq 3}\left(Z^{l_{1}} \tilde{f}_{i j}\right) Z^{l_{2}}\left(\partial_{i j} \dot{\Phi}\right)\right)\right\} \\
& -\frac{3(\gamma-5) L}{2 R(t)} \sum_{i=1}^{3} \partial_{i} \dot{\Phi} Z^{2} \partial_{i} \dot{\Phi}-\frac{3(\gamma-5)}{R(t)} \sum_{i=1}^{3}\left(\left[Z, \partial_{i}\right] \dot{\Phi}\right)^{2} \\
K_{32}^{2}= & -\frac{3(\gamma-5)}{R(t)} \sum_{i=1}^{3}\left(\partial_{i} Z \dot{\Phi}\right)^{2} .
\end{aligned}
$$

Therefore, it follows from the expressions of $\tilde{f}_{i j}, \tilde{f}_{0 i}, \tilde{f}_{0}$ and (5.1) that

$$
\begin{aligned}
\left|K_{31}^{2}\right| \leq C & \left(R(t)^{-3(\gamma-1)-1+\delta} \sum_{0 \leq l \leq 2}\left|\nabla_{x} Z^{l} \dot{\Phi}\right|+R(t)^{-\frac{3(\gamma-1)}{2}-1} \sum_{0 \leq l \leq 2}\left|D_{t} Z^{l} \dot{\Phi}\right|\right. \\
& \left.+R(t)^{-\frac{3(\gamma-1)-\delta}{2}} \sum_{i=1}^{3}\left|Z \partial_{i} D_{t} \dot{\Phi}\right|+R(t)^{-3(\gamma-1)+\frac{\delta}{2}}\left|Z \nabla_{x}^{2} \dot{\Phi}\right|\right) .
\end{aligned}
$$

This implies

$$
\begin{aligned}
& \int_{\Omega_{T}}\left|K_{31}^{2} \cdot \mathcal{M} Z^{2} \dot{\Phi}\right| d t d x \\
& \leq C \varepsilon \sum_{l=0}^{2} \int_{\Omega_{T}}\left(R(t)^{\mu-1-\delta+2 l}\left(\nabla_{t, x}^{l} D_{t} \dot{\Phi}\right)^{2}+R(t)^{\mu-1-3(\gamma-1)+2 l}\left(\nabla_{t, x}^{l} \nabla_{x} \dot{\Phi}\right)^{2}\right) d t d x .
\end{aligned}
$$


Next, we deal with $\int_{\Omega_{T}}\left|K_{32}^{2} \cdot \mathcal{M} Z^{2} \dot{\Phi}\right| d t d x$. By Hölder inequality and Lemma 2.4, we have for $0<\delta \leq \gamma-1$ that

$$
\begin{aligned}
& \left|R(t)^{\mu-1}\left(\nabla_{x} Z \dot{\Phi}\right)^{2} D_{t} Z^{2} \dot{\Phi}\right|_{L^{1}\left(\Omega_{T}\right)} \\
& \leq C\left|R(t)^{\frac{2 \mu+5-3 \gamma-\delta}{4}} \nabla_{x} Z \dot{\Phi}\right|_{L^{4}\left(\Omega_{t}\right)}^{2}\left|R(t)^{\frac{\mu-1-\delta}{2}} D_{t} Z \dot{\Phi}\right|_{L^{2}\left(\Omega_{T}\right)} \\
& \leq C \varepsilon \sum_{l=0}^{2}\left(\left|R(t)^{\frac{\mu-1-\delta+2 l}{2}} \nabla_{t, x}^{l} D_{t} \dot{\Phi}\right|_{L^{2}}+\left|R(t)^{\frac{\mu-1-3(\gamma-1)+2 l}{2}} \nabla_{t, x}^{l} \nabla_{x} \dot{\Phi}\right|_{L^{2}}\right)\left|R(t)^{\frac{\mu-1-\delta}{2}} D_{t} Z^{2} \dot{\Phi}\right|_{L^{2}}
\end{aligned}
$$

(Applying Lemma 2.4 (iii) for $\dot{\Phi}$ )

$$
\leq C \varepsilon \sum_{l=0}^{2}\left(\left|R(t)^{\frac{\mu-1-\delta+2 l}{2}} \nabla_{t, x}^{l} D_{t} \dot{\Phi}\right|_{L^{2}}^{2}+\left|R(t)^{\frac{\mu-1-3(\gamma-1)+2 l}{2}} \nabla_{t, x}^{l} \nabla_{x} \dot{\Phi}\right|_{L^{2}}^{2}\right),
$$

which yields

$\int_{\Omega_{T}}\left|K_{32}^{2} \cdot \mathcal{M} Z^{2} \dot{\Phi}\right| d t d x \leq C \varepsilon \sum_{l=0}^{2} \int_{\Omega_{T}}\left(R(t)^{\mu-1-\delta+2 l}\left(\nabla_{t, x}^{l} D_{t} \dot{\Phi}\right)^{2}+R(t)^{\mu-1-3(\gamma-1)+2 l}\left(\nabla_{t, x}^{l} \nabla_{x} \dot{\Phi}\right)^{2}\right) d t d x$

Combining (5.47) and (5.49) gives

$\int_{\Omega_{T}}\left|K_{3}^{2} \cdot \mathcal{M} Z^{2} \dot{\Phi}\right| d t d x \leq C \varepsilon \sum_{l=0}^{2} \int_{\Omega_{T}}\left(R(t)^{\mu-1-\delta+2 l}\left(\nabla_{t, x}^{l} D_{t} \dot{\Phi}\right)^{2}+R(t)^{\mu-1-3(\gamma-1)+2 l}\left(\nabla_{t, x}^{l} \nabla_{x} \dot{\Phi}\right)^{2}\right) d t d x$.

Next, we consider the case of $m=3$. Due to the lack of $L^{\infty}$ assumptions on the third order derivatives of $\dot{\Phi}$ in (5.1), we will decompose $K_{3}^{3}$ as follows

$$
K_{3}^{3}=K_{31}^{3}+K_{32}^{3}
$$

where $K_{31}^{3}$ is linear with respect to the third and fourth order derivatives of $\dot{\Phi}, K_{32}^{3}$ contains the products of two third order derivatives of $\dot{\Phi} . K_{31}^{3}$ and $K_{32}^{3}$ have the following expressions:

$$
\begin{aligned}
K_{31}^{3} & =\sum_{1 \leq l \leq 2} C_{l 2}\left\{\sum_{l_{1}+l_{2}=l, l_{1} \geq 1} \tilde{C}_{l_{1} l_{2}}\left(\sum_{i=1}^{3}\left(Z^{l_{1}} \tilde{f}_{0 i}\right) Z^{l_{2}}\left(\partial_{i} D_{t} \dot{\Phi}\right)+\sum_{1 \leq i, j \leq 3}\left(Z^{l_{1}} \tilde{f}_{i j}\right) Z^{l_{2}}\left(\partial_{i j}^{2} \dot{\Phi}\right)\right)\right\} \\
& +C_{33} \sum_{\left(l_{1}, l_{2}\right)=(1,2) \text { or }(3,0)} \tilde{C}_{l_{1} l_{2}}\left(\sum_{i=1}^{3}\left(Z^{l_{1}} \tilde{f}_{0 i}\right) Z^{l_{2}}\left(\partial_{i} D_{t} \dot{\Phi}\right)+\sum_{1 \leq i, j \leq 3}\left(Z^{l_{1}} \tilde{f}_{i j}\right) Z^{l_{2}}\left(\partial_{i j}^{2} \dot{\Phi}\right)\right) \\
& +C_{33} C_{21}\left(\sum_{i=1}^{3} Z^{2} \tilde{f}_{0 i}\left[Z, \partial_{i}\right] D_{t} \dot{\Phi}+\sum_{i, j=1}^{3}\left(Z^{2} \tilde{f}_{i j}\left[Z, \partial_{i j}^{2}\right] \dot{\Phi}-2 Z \partial_{i} \dot{\Phi} Z \partial_{j} \dot{\Phi} \partial_{i j}^{2} Z \dot{\Phi}\right)\right. \\
& \left.-2 \sum_{i=1}^{3}\left[Z^{2}, \partial_{i}\right] \dot{\Phi} \partial_{i} Z D_{t} \dot{\Phi}-(\gamma-1) \sum_{i, j=1}^{3}\left(\partial_{j} \dot{\Phi}\left[Z^{2}, \partial_{j}\right] \dot{\Phi} \partial_{i}^{2} Z \dot{\Phi}+\left(Z \partial_{j} \dot{\Phi}\right)^{2} \partial_{i}^{2} Z \dot{\Phi}\right)\right) \\
& -\frac{6(\gamma-5) L}{R(t)} \sum_{i=1}^{3}\left[Z, \partial_{i}\right] \dot{\Phi} Z^{2} \partial_{i} \dot{\Phi},
\end{aligned}
$$


and

$$
\begin{aligned}
K_{32}^{3}= & -C_{33} \tilde{C}_{21}\left(-2 \sum_{i=1}^{3} \partial_{i} Z^{2} \dot{\Phi} \partial_{i} Z D_{t} \dot{\Phi}-2 \sum_{1 \leq i, j \leq 3} \partial_{i} Z^{2} \dot{\Phi} \partial_{j} \dot{\Phi} \partial_{i j}^{2} Z \dot{\Phi}\right. \\
& \left.-(\gamma-1)\left(D_{t} Z^{2} \dot{\Phi}+\sum_{j=1}^{3} \partial_{j} \dot{\Phi} \partial_{j} Z^{2} \dot{\Phi}\right) \sum_{i=1}^{3} \partial_{i}^{2} Z \dot{\Phi}\right)-\frac{6(\gamma-5) L}{R(t)} \sum_{i=1}^{3} \partial_{i} Z \dot{\Phi} Z^{2} \partial_{i} \dot{\Phi}
\end{aligned}
$$

Then it follows from the expressions of $\tilde{f}_{i j}, \tilde{f}_{0 i}, \tilde{f}_{0}$ and (5.1) that

$$
\begin{aligned}
\left|K_{31}^{3}\right| \leq C \varepsilon & \left(R(t)^{-3(\gamma-1)-1+\delta} \sum_{0 \leq l \leq 3}\left|\nabla_{x} Z^{l} \dot{\Phi}\right|+R(t)^{-\frac{3(\gamma-1)+\delta}{2}-1} \sum_{0 \leq l \leq 3}\left|D_{t} Z^{l} \dot{\Phi}\right|\right. \\
& \left.+R(t)^{-\frac{3(\gamma-1)-\delta}{2}} \sum_{0 \leq l \leq 2} \sum_{i=1}^{3}\left|Z^{l} \partial_{i} D_{t} \dot{\Phi}\right|+R(t)^{-3(\gamma-1)+\delta} \sum_{0 \leq l \leq 2}\left|Z^{l} \nabla_{x}^{2} \dot{\Phi}\right|\right)
\end{aligned}
$$

which gives

$$
\begin{aligned}
& \int_{\Omega_{T}}\left|K_{31}^{3} \cdot \mathcal{M} Z^{2} \dot{\Phi}\right| d t d x \\
& \leq C \varepsilon \int_{\Omega_{T}} \sum_{l=0}^{2}\left(R(t)^{\mu-1-\delta+2 l}\left(\nabla_{t, x}^{l} D_{t} \dot{\Phi}\right)^{2}+R(t)^{\mu-1-3(\gamma-1)+2 l}\left(\nabla_{t, x}^{l} \nabla_{x} \dot{\Phi}\right)^{2}\right) d t d x \\
& \quad+C \varepsilon \int_{\Omega_{T}}\left(R(t)^{\mu+5-\delta}\left(\nabla_{t, x}^{3} D_{t} \dot{\Phi}\right)^{2}+R(t)^{\mu+5-3(\gamma-1)-\delta}\left(\nabla_{t, x}^{3} \nabla_{x} \dot{\Phi}\right)^{2}\right) d t d x .
\end{aligned}
$$

We now turn to estimate $\int_{\Omega_{T}}\left|K_{32}^{3} \cdot \mathcal{M} S_{0}^{3} \dot{\Phi}\right| d t d x$. Again, to overcome the lack of weighted $L^{\infty}$ estimates of $\left|\nabla_{t, x}^{3} \dot{\Phi}\right|$ in $K_{32}^{3}$, we use the interpolation inequalities in Corollary 2.1 and Lemma 2.4. In fact, by (5.1) and the expression of $K_{32}^{3}$, it suffices to estimate the following typical terms in $\int_{D_{T}}\left|K_{32}^{3} \cdot \mathcal{M} Z^{3} \dot{\Phi}\right| d t d x$ :

(A) Estimate on $\left|R(t)^{\mu} \partial_{i} Z^{2} \dot{\Phi} \partial_{i} Z D_{t} \dot{\Phi} D_{t} Z^{3} \dot{\Phi}\right|_{L^{1}\left(\Omega_{T}\right)}$ •

$$
\begin{aligned}
& \left|R(t)^{\mu} \partial_{i} Z^{2} \dot{\Phi} \partial_{i} Z D_{t} \dot{\Phi} D_{t} Z^{3} \dot{\Phi}\right|_{L^{1}\left(\Omega_{T}\right)} \\
& \leq C\left|R(t)^{\frac{3 \gamma-5-\delta}{2}} \partial_{i} Z^{2} \dot{\Phi}\right|_{L^{4}}\left|R(t)^{\frac{6 \gamma-6-\delta}{2}} \partial_{i} Z D_{t} \dot{\Phi}\right|_{L^{4}}\left|R(t)^{\frac{\mu-1-\delta}{2}} D_{t} Z^{3} \dot{\Phi}\right|_{L^{2}} \\
& \leq C \varepsilon\left(\sum_{k=0}^{1}\left|R(t)^{\frac{\mu+4-3 \gamma}{2}}-k \nabla_{t, x}^{2-k} Z \dot{\Phi}\right|_{L^{2}}^{\frac{1}{2}}+\sum_{k=0}^{2}\left|R(t)^{\frac{3 \gamma-3-\delta}{2}-k} \nabla_{t, x}^{3-k} Z \dot{\Phi}\right|_{L^{2}}^{\frac{1}{2}}\right)\left|R(t)^{\frac{\mu-1-\delta}{2}} D_{t} Z^{3} \dot{\Phi}\right|_{L^{2}}
\end{aligned}
$$

(Applying Lemma 2.4 (v)-(vi) for $\dot{\Phi}$ )

$$
\begin{aligned}
\leq & C \varepsilon \int_{\Omega_{T}} \sum_{l=0}^{2}\left(R(t)^{\mu-1-\delta+2 l}\left(\nabla_{t, x}^{l} D_{t} \dot{\Phi}\right)^{2}+R(t)^{\mu-1-3(\gamma-1)+2 l}\left(\nabla_{t, x}^{l} \nabla_{x} \dot{\Phi}\right)^{2}\right) d t d x \\
& +C \varepsilon \int_{\Omega_{T}}\left(R(t)^{\mu+5-\delta}\left(\nabla_{t, x}^{3} D_{t} \dot{\Phi}\right)^{2}+R(t)^{\mu+5-3(\gamma-1)-\delta}\left(\nabla_{t, x}^{3} \nabla_{x} \dot{\Phi}\right)^{2}\right) d t d x .
\end{aligned}
$$

(B) Estimate on $\left|R(t)^{\mu} \partial_{i} Z^{2} \dot{\Phi} \partial_{j} \dot{\Phi} \partial_{i j}^{2} Z \dot{\Phi} D_{t} Z^{3} \dot{\Phi}\right|_{L^{1}\left(\Omega_{T}\right)}$. $\left|R(t)^{\mu} \partial_{i} Z^{2} \dot{\Phi} \partial_{j} \dot{\Phi} \partial_{i j}^{2} Z \dot{\Phi} D_{t} Z^{3} \dot{\Phi}\right|_{L^{1}\left(\Omega_{T}\right)}$ 
32

$$
\begin{aligned}
\leq & C \varepsilon\left|R(t)^{\frac{3 \gamma-5-\delta}{2}} \partial_{i} Z^{2} \dot{\Phi}\right|_{L^{4}}\left|R(t)^{\frac{3 \gamma-3-\delta}{2}} \partial_{i j}^{2} Z \dot{\Phi}\right|_{L^{4}}\left|R(t)^{\frac{\mu-1-\delta}{2}} D_{t} Z^{3} \dot{\Phi}\right|_{L^{2}} \quad \text { (By assumption (5.1)) } \\
\leq & C \varepsilon\left(\sum_{k=0}^{1}\left|R(t)^{\frac{\mu+4-3 \gamma}{2}-k} \nabla_{t, x}^{2-k} Z \dot{\Phi}\right|_{L^{2}}^{\frac{1}{2}}+\sum_{k=0}^{2}\left|R(t)^{\frac{3 \gamma-3-\delta}{2}-k} \nabla_{t, x}^{3-k} Z \dot{\Phi}\right|_{L^{2}}^{\frac{1}{2}}\right)\left|R(t)^{\frac{\mu-1-\delta}{2}} D_{t} Z^{3} \dot{\Phi}\right|_{L^{2}} \\
& \text { (Applying Lemma 2.4 (iv) and (vii) for } \dot{\Phi}) \\
\leq & C \varepsilon \int_{\Omega_{T}} \sum_{l=0}^{2}\left(R(t)^{\mu-1-\delta+2 l}\left(\nabla_{t, x}^{l} D_{t} \dot{\Phi}\right)^{2}+R(t)^{\mu-1-3(\gamma-1)+2 l}\left(\nabla_{t, x}^{l} \nabla_{x} \dot{\Phi}\right)^{2}\right) d t d x \\
& +C \varepsilon \int_{\Omega_{T}}\left(R(t)^{\mu+5-\delta}\left(\nabla_{t, x}^{3} D_{t} \dot{\Phi}\right)^{2}+R(t)^{\mu+5-3(\gamma-1)-\delta}\left(\nabla_{t, x}^{3} \nabla_{x} \dot{\Phi}\right)^{2}\right) d t d x .
\end{aligned}
$$

(C) Estimate on $\left|R(t)^{\mu} D_{t} Z^{2} \dot{\Phi} \partial_{i}^{2} Z \dot{\Phi} D_{t} Z^{3} \dot{\Phi}\right|_{L^{1}\left(\Omega_{T}\right)}$.

$$
\begin{aligned}
& \left|R(t)^{\mu} D_{t} Z^{2} \dot{\Phi} \partial_{i}^{2} Z \dot{\Phi} D_{t} Z^{3} \dot{\Phi}\right|_{L^{1}\left(\Omega_{T}\right)} \\
& \leq C\left|R(t)^{\frac{6 \gamma-8-\delta}{2}} D_{t} Z^{2} \dot{\Phi}\right|_{L^{4}}\left|R(t)^{\frac{3 \gamma-3-\delta}{2}} \partial_{i}^{2} Z \dot{\Phi}\right|_{L^{4}}\left|R(t)^{\frac{\mu-1-\delta}{2}} D_{t} Z^{3} \dot{\Phi}\right|_{L^{2}} \\
& \leq C \varepsilon\left(\sum_{k=0}^{2}\left|R(t)^{\frac{3 \gamma-3-\delta}{2}-k} \nabla_{t, x}^{3-k} Z \dot{\Phi}\right|_{L^{2}}^{\frac{1}{2}}+\sum_{k=0}^{2}\left|R(t)^{\frac{6 \gamma-6-\delta}{2}-k} \nabla_{t, x}^{2-k} Z D_{t} \dot{\Phi}\right|_{L^{2}}^{\frac{1}{2}}\right)\left|R(t)^{\frac{\mu-1-\delta}{2}} D_{t} Z^{3} \dot{\Phi}\right|_{L^{2}} \\
& \text { (Applying Lemma } 2.4 \text { (iv) and (vii) for } \dot{\Phi} \text { ) } \\
& \leq C \varepsilon \int_{\Omega_{T}} \sum_{l=0}^{2}\left(R(t)^{\mu-1-\delta+2 l}\left(\nabla_{t, x}^{l} D_{t} \dot{\Phi}\right)^{2}+R(t)^{\mu-1-3(\gamma-1)+2 l}\left(\nabla_{t, x}^{l} \nabla_{x} \dot{\Phi}\right)^{2}\right) d t d x \\
& +C \varepsilon \int_{\Omega_{T}}\left(R(t)^{\mu+5-\delta}\left(\nabla_{t, x}^{3} D_{t} \dot{\Phi}\right)^{2}+R(t)^{\mu+5-3(\gamma-1)-\delta}\left(\nabla_{t, x}^{3} \nabla_{x} \dot{\Phi}\right)^{2}\right) d t d x .
\end{aligned}
$$

(D) Estimate on $\left|R(t)^{\mu-1} \partial_{i} Z \dot{\Phi} Z^{2} \partial_{i} \dot{\Phi} D_{t} Z^{3} \dot{\Phi}\right|_{L^{1}\left(\Omega_{T}\right)}$ •

$$
\begin{aligned}
& \left|R(t)^{\mu-1} \partial_{i} Z \dot{\Phi} Z^{2} \partial_{i} \dot{\Phi} D_{t} Z^{3} \dot{\Phi}\right|_{L^{1}\left(\Omega_{T}\right)} \\
& =\left|R(t)^{\frac{5 \delta-3(\gamma-1)}{4}} \cdot R(t)^{\frac{9 \gamma-13-\delta}{4}} \partial_{i} Z \dot{\Phi} \cdot R(t)^{\frac{3 \gamma-5-\delta}{2}} Z^{2} \partial_{i} \dot{\Phi} \cdot R(t)^{\frac{\mu-1-\delta}{2}} D_{t} Z^{3} \dot{\Phi}\right|_{L^{1}\left(\Omega_{T}\right)} \\
& \text { (here we have used the restriction of } \delta \leq \frac{3}{5}(\gamma-1) \text { ) } \\
& \leq C\left|R(t)^{\frac{9 \gamma-13-\delta}{4}} \partial_{i} Z \dot{\Phi}\right|_{L^{4}}\left|R(t)^{\frac{3 \gamma-5-\delta}{2}} Z^{2} \partial_{i} \dot{\Phi}\right|_{L^{4}}\left|R(t)^{\frac{\mu-1-\delta}{2}} D_{t} Z^{3} \dot{\Phi}\right|_{L^{2}} \\
& \leq C \varepsilon\left(\sum_{k=0}^{1}\left|R(t)^{\frac{\mu+4-3 \gamma}{2}-k} \nabla_{t, x}^{2-k} Z \dot{\Phi}\right|_{L^{2}}^{\frac{1}{2}}+\sum_{k=0}^{2}\left|R(t)^{\frac{3 \gamma-3-\delta}{2}-k} \nabla_{t, x}^{3-k} Z \dot{\Phi}\right|_{L^{2}}^{\frac{1}{2}}\right)\left|R(t)^{\frac{\mu-1-\delta}{2}} D_{t} Z^{3} \dot{\Phi}\right|_{L^{2}} \\
& \text { (Applying Lemma } 2.4 \text { (iii) and (vi) for } \dot{\Phi} \text { ) } \\
& \leq C \varepsilon \int_{\Omega_{T}} \sum_{l=0}^{2}\left(R(t)^{\mu-1-\delta+2 l}\left(\nabla_{t, x}^{l} D_{t} \dot{\Phi}\right)^{2}+R(t)^{\mu-1-3(\gamma-1)+2 l}\left(\nabla_{t, x}^{l} \nabla_{x} \dot{\Phi}\right)^{2}\right) d t d x \\
& +C \varepsilon \int_{\Omega_{T}}\left(R(t)^{\mu+5-\delta}\left(\nabla_{t, x}^{3} D_{t} \dot{\Phi}\right)^{2}+R(t)^{\mu+5-3(\gamma-1)-\delta}\left(\nabla_{t, x}^{3} \nabla_{x} \dot{\Phi}\right)^{2}\right) d t d x .
\end{aligned}
$$


Substituting (5.53)-(5.56) into $\int_{\Omega_{T}}\left|K_{32}^{3} \cdot \mathcal{M} Z^{3} \dot{\Phi}\right| d t d x$ yields

$$
\begin{aligned}
& \int_{\Omega_{T}}\left|K_{32}^{3} \cdot \mathcal{M} Z^{3} \dot{\Phi}\right| d t d x \\
& \leq C \varepsilon \int_{\Omega_{T}} \sum_{l=0}^{2}\left(R(t)^{\mu-1-\delta+2 l}\left(\nabla_{t, x}^{l} D_{t} \dot{\Phi}\right)^{2}+R(t)^{\mu-1-3(\gamma-1)+2 l}\left(\nabla_{t, x}^{l} \nabla_{x} \dot{\Phi}\right)^{2}\right) d t d x \\
& \quad+C \varepsilon \int_{\Omega_{T}}\left(R(t)^{\mu+5-\delta}\left(\nabla_{t, x}^{3} D_{t} \dot{\Phi}\right)^{2}+R(t)^{\mu+5-3(\gamma-1)-\delta}\left(\nabla_{t, x}^{3} \nabla_{x} \dot{\Phi}\right)^{2}\right) d t d x .
\end{aligned}
$$

Then putting (5.44)-(5.46), (5.51)-(5.52) and (5.57) into (5.8) yields (5.4) and (5.6) for the case $S^{m}=Z^{m}$.

Case (3) $\quad S^{m}=S_{0}^{l_{1}} Z^{l_{2}}\left(1 \leq l_{1}, l_{2} \leq m-1, l_{1}+l_{2}=m\right)$.

Since the estimation for this case is similar to the Case (1) and Case (2), we omit the detail.

Combining the Cases (1)-(3), we complete the proof of Lemma 5.1.

Based on Lemma 5.1, we now derive a series of estimates on the higher order derivatives of $\dot{\Phi}$. For convenience, set $S_{1}=r \partial_{r}=\sum_{i=1}^{3} x_{i} \partial_{i}$.

Lemma 5.2 (The second order radial derivative estimates) Under the assumptions of Theorem 5.1, we have

$$
\begin{aligned}
& R(T)^{\mu} \int_{S_{T}}\left(D_{t} S_{1} \dot{\Phi}\right)^{2} d S+R(T)^{\mu-3(\gamma-1)} \int_{S_{T}}\left(\nabla_{x} S_{1} \dot{\Phi}\right)^{2} d S \\
& \quad+\int_{\Omega_{T}}\left(R(t)^{\mu-1-\delta}\left(D_{t} S_{1} \dot{\Phi}\right)^{2}+R(t)^{\mu-1-3(\gamma-1)}\left(\nabla_{x} S_{1} \dot{\Phi}\right)^{2}\right) d t d x \\
& \leq C \varepsilon^{2}+C \varepsilon \int_{\Omega_{T}} \sum_{l=0}^{1}\left(R(t)^{\mu-1-\delta+2 l}\left(\nabla_{t, x}^{l} D_{t} \dot{\Phi}\right)^{2}+R(t)^{\mu-1-3(\gamma-1)+2 l}\left(\nabla_{t, x}^{l} \nabla_{x} \dot{\Phi}\right)^{2}\right) d t d x \\
& \quad+C \varepsilon\left(\int_{\Omega_{T}} \sum_{l=0}^{1}\left(R(t)^{\mu-1-\delta+2 l}\left(\nabla_{t, x}^{l} D_{t} \dot{\Phi}\right)^{2}+R(t)^{\mu-1-3(\gamma-1)+2 l}\left(\nabla_{t, x}^{l} \nabla_{x} \dot{\Phi}\right)^{2}\right) d t d x\right)^{\frac{1}{2}} .
\end{aligned}
$$

Remark 5.3 Lemma 5.2, together with Lemma 5.1 for $m=1$ yield

$$
\begin{aligned}
& \sum_{0 \leq l_{1}+l_{2} \leq 1}\left(R(T)^{\mu} \int_{S_{T}}\left(D_{t} S^{l_{1}} S_{1}^{l_{2}} \dot{\Phi}\right)^{2} d S+R(T)^{\mu-3(\gamma-1)} \int_{S_{T}}\left(\nabla_{x} S^{l_{1}} S_{1}^{l_{2}} \dot{\Phi}\right)^{2} d S\right) \\
+ & \sum_{0 \leq l_{1}+l_{2} \leq 1} \int_{\Omega_{T}}\left(R(t)^{\mu-1-\delta}\left(D_{t} S^{l_{1}} S_{1}^{l_{2}} \dot{\Phi}\right)^{2}+R(t)^{\mu-1-3(\gamma-1)}\left(\nabla_{x} S^{l_{1}} S_{1}^{l_{2}} \dot{\Phi}\right)^{2}\right) d t d x \\
\leq & C \varepsilon^{2}+C \varepsilon \int_{\Omega_{T}} \sum_{l=0}^{1}\left(R(t)^{\mu-1-\delta+2 l}\left(\nabla_{t, x}^{l} D_{t} \dot{\Phi}\right)^{2}+R(t)^{\mu-1-3(\gamma-1)+2 l}\left(\nabla_{t, x}^{l} \nabla_{x} \dot{\Phi}\right)^{2}\right) d t d x \\
+ & C \varepsilon\left(\int_{\Omega_{T}} \sum_{l=0}^{1}\left(R(t)^{\mu-1-\delta+2 l}\left(\nabla_{t, x}^{l} D_{t} \dot{\Phi}\right)^{2}+R(t)^{\mu-1-3(\gamma-1)+2 l}\left(\nabla_{t, x}^{l} \nabla_{x} \dot{\Phi}\right)^{2}\right) d t d x\right)^{\frac{1}{2}} .
\end{aligned}
$$


Proof of Lemma 5.2. Noting $\mathcal{L} S_{1} \dot{\Phi}=S_{1} \mathcal{L} \dot{\Phi}-2 \hat{c}^{2} \Delta \dot{\Phi}$, then it follows from Theorem 4.1 that

$$
\begin{aligned}
& R(T)^{\mu} \int_{S_{T}}\left(D_{t} S_{1} \dot{\Phi}\right)^{2} d S+R(T)^{\mu-3(\gamma-1)} \int_{S_{T}}\left(\nabla_{x} S_{1} \dot{\Phi}\right)^{2} d S \\
& +\int_{\Omega_{T}}\left(R(t)^{\mu-1-\delta}\left(D_{t} S_{1} \dot{\Phi}\right)^{2}+R(t)^{\mu-1-3(\gamma-1)}\left(\nabla_{x} S_{1} \dot{\Phi}\right)^{2}\right) d t d x \\
\leq & C \varepsilon^{2}+C \int_{\Omega_{T}}\left(S_{1} \dot{f}-2 \hat{c}^{2} \Delta \dot{\Phi}\right) \cdot \mathcal{M} S_{1} \dot{\Phi} d t d x .
\end{aligned}
$$

Since $S_{1}$ and $Z$ are expressed in terms of $x_{i} \partial_{j}(1 \leq i, j \leq 3)$, similar to the proofs of (5.43)-(5.45) and (5.47), we have

$$
\begin{aligned}
& \left|\int_{\Omega_{T}} S_{1} \dot{f} \cdot \mathcal{M} S_{1} \dot{\Phi} d t d x\right| \\
& \leq C \varepsilon^{2}+C \varepsilon\left(R(T)^{\mu} \int_{S_{T}}\left(D_{t} S_{1} \dot{\Phi}\right)^{2} d S+R(T)^{\mu-3(\gamma-1)} \int_{S_{T}}\left(\nabla_{x} S_{1} \dot{\Phi}\right)^{2} d S\right) \\
& \quad+C \varepsilon \int_{\Omega_{T}} \sum_{l=0}^{1}\left(R(t)^{\mu-1-\delta+2 l}\left(\nabla_{t, x}^{l} D_{t} \dot{\Phi}\right)^{2}+R(t)^{\mu-1-3(\gamma-1)+2 l}\left(\nabla_{t, x}^{l} \nabla_{x} \dot{\Phi}\right)^{2}\right) d t d x .
\end{aligned}
$$

In addition, thanks to (5.4) for the case of $S^{m}=S_{0}$, it follows

$$
\begin{aligned}
& \int_{\Omega_{T}}\left|\hat{c}^{2} \Delta \dot{\Phi} \cdot \mathcal{M} S_{1} \dot{\Phi}\right| d t d x \\
& \leq C \int_{\Omega_{T}} R(t)^{\mu-3(\gamma-1)}\left|\Delta \dot{\Phi} \nabla_{x} S_{0} \dot{\Phi}\right| d t d x \\
& \leq C\left(\int_{\Omega_{T}} R(t)^{\mu+1-3(\gamma-1)}(\Delta \dot{\Phi})^{2} d t d x\right)^{\frac{1}{2}}\left(\int_{\Omega_{T}} R(t)^{\mu-1-3(\gamma-1)}\left(\nabla_{x} S_{0} \dot{\Phi}\right)^{2} d t d x\right)^{\frac{1}{2}} \\
& \leq C \varepsilon^{2}+C \varepsilon\left(\sum_{l=0}^{1} \int_{\Omega_{T}} R(t)^{\mu-1-\delta+2 l}\left(\nabla_{t, x}^{l} D_{t} \dot{\Phi}\right)^{2}+R(t)^{\mu-1-3(\gamma-1)+2 l}\left(\nabla_{t, x}^{l} \nabla_{x} \dot{\Phi}\right)^{2} d t d x\right)^{\frac{1}{2}} .
\end{aligned}
$$

Finally, substituting (5.60)-(5.61) into (5.59) yields (5.58), and this completes the proof of the lemma.

Lemma 5.3 (The third order radial derivative estimates) Under the assumptions of Theorem 5.1, we have

$$
\begin{aligned}
& R(T)^{\mu} \int_{S_{T}}\left(D_{t} S_{1}^{2} \dot{\Phi}\right)^{2} d S+R(T)^{\mu-3(\gamma-1)} \int_{S_{T}}\left(\nabla_{x} S_{1}^{2} \dot{\Phi}\right)^{2} d S \\
& +\int_{\Omega_{T}}\left(R(t)^{\mu-1-\delta}\left(D_{t} S_{1}^{2} \dot{\Phi}\right)^{2}+R(t)^{\mu-1-3(\gamma-1)}\left(\nabla_{x} S_{1}^{2} \dot{\Phi}\right)^{2}\right) d t d x \\
\leq & C \varepsilon^{2}+C \varepsilon \int_{\Omega_{T}}\left(\sum_{l=0}^{3} R(t)^{\mu-1-\delta+2 l}\left(\nabla_{t, x}^{l} D_{t} \dot{\Phi}\right)^{2}+\sum_{l=0}^{2} R(t)^{\mu-1-3(\gamma-1)+2 l}\left(\nabla_{t, x}^{l} \nabla_{x} \dot{\Phi}\right)^{2}\right) d t d x \\
& +C \varepsilon\left(\int_{\Omega_{T}} R(t)^{\mu+3-\delta}\left(\nabla_{t, x}^{2} D_{t} \dot{\Phi}\right)^{2}+R(t)^{\mu+3-3(\gamma-1)}\left(\nabla_{t, x}^{2} \nabla_{x} \dot{\Phi}\right)^{2} d t d x\right)^{\frac{1}{2}} .
\end{aligned}
$$


Remark 5.4 Under the assumptions of Theorem 5.1, as in the proof of Lemma 5.1, we have

$$
\begin{aligned}
& R(T)^{\mu} \int_{S_{T}}\left(D_{t} S S_{1} \dot{\Phi}\right)^{2} d S+R(T)^{\mu-3(\gamma-1)} \int_{S_{T}}\left(\nabla_{x} S S_{1} \dot{\Phi}\right)^{2} d S \\
& +\int_{\Omega_{T}}\left(R(t)^{\mu-1-\delta}\left(D_{t} S S_{1} \dot{\Phi}\right)^{2}+R(t)^{\mu-1-3(\gamma-1)}\left(\nabla_{x} S S_{1} \dot{\Phi}\right)^{2}\right) d t d x \\
\leq & C \varepsilon^{2}+C \varepsilon \int_{\Omega_{T}} \sum_{l=0}^{2}\left(R(t)^{\mu-1-\delta+2 l}\left(\nabla_{t, x}^{l} D_{t} \dot{\Phi}\right)^{2}+R(t)^{\mu-1-3(\gamma-1)+2 l}\left(\nabla_{t, x}^{l} \nabla_{x} \dot{\Phi}\right)^{2}\right) d t d x \\
+ & C \varepsilon\left(\sum_{l=0}^{2} \int_{\Omega_{T}} R(t)^{\mu-1-\delta+2 l}\left(\nabla_{t, x}^{l} D_{t} \dot{\Phi}\right)^{2}+R(t)^{\mu-1-3(\gamma-1)+2 l}\left(\nabla_{t, x}^{l} \nabla_{x} \dot{\Phi}\right)^{2} d t d x\right)^{\frac{1}{2}} .
\end{aligned}
$$

This, together with (5.62) and Lemma 5.1 for $m=2$ and Remark 2.2, yields the following estimate

$$
\begin{aligned}
& \sum_{0 \leq l_{1}+l_{2} \leq 2}\left(R(T)^{\mu} \int_{S_{T}}\left(D_{t} S^{l_{1}} S_{1}^{l_{2}} \dot{\Phi}\right)^{2} d S+R(T)^{\mu-3(\gamma-1)} \int_{S_{T}}\left(\nabla_{x} S^{l_{1}} S_{1}^{l_{2}} \dot{\Phi}\right)^{2} d S\right) \\
& +\sum_{0 \leq l_{1}+l_{2} \leq 2} \int_{\Omega_{T}}\left(R(t)^{\mu-1-\delta}\left(D_{t} S^{l_{1}} S_{1}^{l_{2}} \dot{\Phi}\right)^{2}+R(t)^{\mu-1-3(\gamma-1)}\left(\nabla_{x} S^{l_{1}} S_{1}^{l_{2}} \dot{\Phi}\right)^{2}\right) d t d x \\
\leq & C \varepsilon^{2}+C \varepsilon \int_{\Omega_{T}} \sum_{l=0}^{2}\left(R(t)^{\mu-1-\delta+2 l}\left(\nabla_{t, x}^{l} D_{t} \dot{\Phi}\right)^{2}+R(t)^{\mu-1-3(\gamma-1)+2 l}\left(\nabla_{t, x}^{l} \nabla_{x} \dot{\Phi}\right)^{2}\right) d t d x \\
& +C \varepsilon \int_{\Omega_{T}}\left(R(t)^{\mu+5-\delta}\left(\nabla_{t, x}^{3} D_{t} \dot{\Phi}\right)^{2}+R(t)^{\mu+5-3(\gamma-1)-\delta}\left(\nabla_{t, x}^{3} \nabla_{x} \dot{\Phi}\right)^{2}\right) d t d x \\
& +C \varepsilon\left(\int_{\Omega_{T}} \sum_{l=0}^{2}\left(R(t)^{\mu-1-\delta+2 l}\left(\nabla_{t, x}^{l} D_{t} \dot{\Phi}\right)^{2}+R(t)^{\mu-1-3(\gamma-1)+2 l}\left(\nabla_{t, x}^{l} \nabla_{x} \dot{\Phi}\right)^{2}\right) d t d x\right. \\
+ & \left.\int_{\Omega_{T}}\left(R(t)^{\mu+5-\delta}\left(\nabla_{t, x}^{3} D_{t} \dot{\Phi}\right)^{2}+R(t)^{\mu+5-3(\gamma-1)-\delta}\left(\nabla_{t, x}^{3} \nabla_{x} \dot{\Phi}\right)^{2}\right) d t d x\right)^{\frac{1}{2}} .
\end{aligned}
$$

Proof of Lemma 5.3. In order to derive the third order radial derivative estimate, we need to study a higher order boundary condition on $B_{T}$. Differentiating (4.16) with respect to $r$ and applying (5.7) yield on $B_{T}$

$$
S_{1}^{3} \dot{\Phi}-S_{1}^{2} \dot{\Phi}-2 \sum_{i=1}^{3} Z_{i}^{2} \dot{\Phi}-\frac{G}{A}=0
$$

where

$$
\begin{aligned}
A= & \hat{c}^{2}-(\gamma-1) D_{t} \dot{\Phi}-\frac{\gamma-1}{2 R(t)^{2}} \sum_{i=1}^{3}\left(Z_{i} \dot{\Phi}\right)^{2} \\
G= & -\frac{\gamma-1}{R(t)^{2}} \sum_{i=1}^{3}\left(Z_{i} \dot{\Phi}\right)^{2}\left(S_{1}^{2} \dot{\Phi}+\sum_{i=1}^{3} Z_{i}^{2} \dot{\Phi}\right)-\frac{3(\gamma-1) L}{R(t)} \sum_{i=1}^{3}\left(Z_{i} \dot{\Phi}\right)^{2}-\frac{2 L}{R(t)} \sum_{i, j=1}^{3} C_{i j} Z_{i} \dot{\Phi} Z_{j} \dot{\Phi} \\
& -\frac{4}{R(t)^{2}} \sum_{i, j=1}^{3} Z_{i} \dot{\Phi} Z_{j} \dot{\Phi} Z_{i} Z_{j} \dot{\Phi}+\frac{1}{R(t)^{2}} S_{1}^{2} \dot{\Phi} \sum_{i, j=1}^{3} C_{i j} Z_{i} \dot{\Phi} Z_{j} \dot{\Phi}-\frac{4}{R(t)^{2}} \sum_{i, j, k=1}^{3} C_{i j k} Z_{i} \dot{\Phi} Z_{j} \dot{\Phi} Z_{k} \dot{\Phi} .
\end{aligned}
$$


36

For convenience, we rewrite (5.65) as follows

$$
S_{1}^{3} \dot{\Phi}-S_{1}^{2} \dot{\Phi}-2 \sum_{i=1}^{3} Z_{i}^{2} \dot{\Phi}-\chi\left(\frac{r}{R(t)}\right) \frac{G}{A}=0 \quad \text { on } B_{T},
$$

where $\chi(s)$ is a smooth cut-off function

$$
\chi(s)=\left\{\begin{array}{cl}
1, & \text { for } \frac{2}{3} \leq s \leq 1 \\
0, & \text { for } 0 \leq s \leq \frac{1}{3} \\
\text { smooth connection, }, & \text { for } \frac{1}{3} \leq s \leq \frac{2}{3} .
\end{array}\right.
$$

On the other hand, direct computation yields

$$
\mathcal{L}\left(S_{1}^{2}-S_{1}\right) \dot{\Phi}=\left(S_{1}^{2}-S_{1}\right) \mathcal{L} \dot{\Phi}+4 \hat{c}^{2} \Delta S_{1} \dot{\Phi}-2 \hat{c}^{2} \Delta \dot{\Phi} .
$$

As in (4.2), we have

$$
\begin{aligned}
& \int_{\Omega_{T}} \mathcal{L}\left(S_{1}^{2}-S_{1}\right) \dot{\Phi} \cdot \mathcal{M}\left(S_{1}^{2}-S_{1}\right) \dot{\Phi} d t d x \\
& =-\int_{B_{T}} \frac{R(t)^{\mu}}{\sqrt{1+L^{2}}} \hat{c}^{2} a(t) D_{t}\left(S_{1}^{2}-S_{1}\right) \dot{\Phi} \cdot \partial_{r}\left(S_{1}^{2}-S_{1}\right) \dot{\Phi} d S \\
& \quad+\left(\int_{S_{T}}-\int_{S^{0}}\right) \frac{1}{2} R(t)^{\mu} a(t)\left(D_{t}\left(S_{1}^{2}-S_{1}\right) \dot{\Phi}+\hat{c}^{2}\left|\nabla_{x}\left(S_{1}^{2}-S_{1}\right) \dot{\Phi}\right|^{2}\right) d S \\
& \quad+\int_{\Omega_{T}}\left\{\frac{\delta}{2} R(t)^{\mu-1-\delta}\left(D_{t}\left(S_{1}^{2}-S_{1}\right) \dot{\Phi}\right)^{2}+\frac{\gamma}{2} R(t)^{\mu-1-3(\gamma-1)}(L(5-3 \gamma) a(t)\right. \\
& \left.\left.\quad-R(t) a^{\prime}(t)\right)\left|\nabla_{x}\left(S_{1}^{2}-S_{1}\right) \dot{\Phi}\right|^{2}\right\} d t d x .
\end{aligned}
$$

Substituting (5.68) into (5.69), it follows

$$
\begin{aligned}
& R(T)^{\mu} \int_{S_{T}}\left(D_{t}\left(S_{1}^{2}-S_{1}\right) \dot{\Phi}\right)^{2} d S+R(T)^{\mu-3(\gamma-1)} \int_{S_{T}}\left(\nabla_{x}\left(S_{1}^{2}-S_{1}\right) \dot{\Phi}\right)^{2} d S \\
& \quad+\int_{\Omega_{T}}\left(R(t)^{\mu-1-\delta}\left(D_{t}\left(S_{1}^{2}-S_{1}\right) \dot{\Phi}\right)^{2}+R(t)^{\mu-1-3(\gamma-1)}\left(\nabla_{x}\left(S_{1}^{2}-S_{1}\right) \dot{\Phi}\right)^{2}\right) d t d x \\
& \leq C \varepsilon^{2}+\int_{\Omega_{T}}\left(\left(S_{1}^{2}-S_{1}\right) \dot{f}+4 \hat{c}^{2} \Delta S_{1} \dot{\Phi}-2 \hat{c}^{2} \Delta \dot{\Phi}\right) \cdot \mathcal{M}\left(S_{1}^{2}-S_{1}\right) \dot{\Phi} d t d x \\
& \quad+\int_{B_{T}} \frac{R(t)^{\mu}}{\sqrt{1+L^{2}}} \hat{c}^{2} a(t) D_{t}\left(S_{1}^{2}-S_{1}\right) \dot{\Phi} \cdot \partial_{r}\left(S_{1}^{2}-S_{1}\right) \dot{\Phi} d S .
\end{aligned}
$$

In view of (5.66) and divergence theorem, we have

$$
\begin{aligned}
& \int_{B_{T}} \frac{R(t)^{\mu}}{\sqrt{1+L^{2}}} \hat{c}^{2} a(t) D_{t}\left(S_{1}^{2}-S_{1}\right) \dot{\Phi} \cdot \partial_{r}\left(S_{1}^{2}-S_{1}\right) \dot{\Phi} d S \\
& =\int_{\Omega_{T}} R(t)^{\mu} \hat{c}^{2} a(t) \sum_{i=1}^{3} \partial_{i}\left[\partial_{i}\left(D_{t} S_{1} \dot{\Phi}-D_{t} \dot{\Phi}\right)\left(\chi\left(\frac{r}{R(t)}\right) \frac{G}{A}+2 \sum_{i=1}^{3} Z_{i}^{2} \dot{\Phi}\right)\right] d t d x
\end{aligned}
$$




$$
\begin{aligned}
= & \int_{\Omega_{T}} R(t)^{\mu} \hat{c}^{2} a(t)\left[\Delta\left(D_{t} S_{1} \dot{\Phi}-D_{t} \dot{\Phi}\right)\left(\chi\left(\frac{r}{R(t)}\right) \frac{G}{A}+2 \sum_{i=1}^{3} Z_{i}^{2} \dot{\Phi}\right)\right] d t d x \\
& +\int_{\Omega_{T}} R(t)^{\mu} \hat{c}^{2} a(t) \sum_{j=1}^{3} \partial_{j}\left(D_{t} S_{1} \dot{\Phi}-D_{t} \dot{\Phi}\right)\left(\partial_{j}\left(\chi\left(\frac{r}{R(t)}\right) \frac{G}{A}\right)+2 \sum_{i=1}^{3} \partial_{j} Z_{i}^{2} \dot{\Phi}\right) d t d x .
\end{aligned}
$$

And it follows from (5.2) that

$$
\begin{gathered}
\left|\chi\left(\frac{r}{R(t)}\right) \frac{G}{A}\right| \leq C \varepsilon\left(R(t)^{-3(\gamma-1)}\left(\left|S_{1}^{2} \dot{\Phi}\right|+\left|Z^{2} \dot{\Phi}\right|\right)+|Z \dot{\Phi}|\right) \\
\left|\nabla_{x}\left(\chi\left(\frac{r}{R(t)}\right) \frac{G}{A}\right)\right| \leq C \varepsilon\left(R(t)^{-\frac{3(\gamma-1)}{2}-1}\left(\left|S_{1}^{2} \dot{\Phi}\right|+\left|Z^{2} \dot{\Phi}\right|\right)+R(t)^{-3(\gamma-1)}\left(\left|\nabla_{x} S_{1}^{2} \dot{\Phi}\right|+\left|\nabla_{x} Z^{2} \dot{\Phi}\right|\right)\right. \\
\left.+\sum_{0 \leq l \leq 1} R(t)^{l-1}\left|\nabla_{x}^{l} Z \dot{\Phi}\right|+\left|\nabla_{x} S_{0} \dot{\Phi}\right|\right)
\end{gathered}
$$

Combining (5.71)-(5.73), it follows

$$
\begin{aligned}
& \left|\int_{B_{T}} \frac{R(t)^{\mu}}{\sqrt{1+L^{2}}} \hat{c}^{2} a(t) D_{t}\left(S_{1}^{2}-S_{1}\right) \dot{\Phi} \cdot \partial_{r}\left(S_{1}^{2}-S_{1}\right) \dot{\Phi} d S\right| \\
& \leq C \varepsilon^{2}+C \varepsilon \int_{\Omega_{T}}\left(\sum_{l=0}^{3} R(t)^{\mu-1-\delta+2 l}\left(\nabla_{t, x}^{l} D_{t} \dot{\Phi}\right)^{2}+\sum_{l=0}^{2} R(t)^{\mu-1-3(\gamma-1)+2 l}\left(\nabla_{t, x}^{l} \nabla_{x} \dot{\Phi}\right)^{2}\right) d t d x .
\end{aligned}
$$

Then as in (5.61), thanks to (5.63) and (5.8) in the case of $S^{2}=Z S_{0}$, it follows

$$
\begin{aligned}
& \int_{\Omega_{T}}\left(\left|\hat{c}^{2} \Delta S_{1} \dot{\Phi}\right|+\left|\hat{c}^{2} \Delta \dot{\Phi}\right|\right) \cdot\left|\mathcal{M}\left(S_{1}^{2}+S_{1}\right) \dot{\Phi}\right| d t d x \\
\leq & C \varepsilon^{2}+C \varepsilon\left(\int_{\Omega_{T}} R(t)^{\mu+3-\delta}\left(\nabla_{t, x}^{2} D_{t} \dot{\Phi}\right)^{2}+R(t)^{\mu+3-3(\gamma-1)}\left(\nabla_{t, x}^{2} \nabla_{x} \dot{\Phi}\right)^{2} d t d x\right)^{\frac{1}{2}} .
\end{aligned}
$$

Next we deal with $\int_{\Omega_{T}}\left(S_{1}^{2}-S_{1}\right) \dot{f} \cdot \mathcal{M}\left(S_{1}^{2}-S_{1}\right) \dot{\Phi} d t d x$. Similar to (5.12), we have

$$
\left(S_{1}^{2}-S_{1}\right) \dot{f}=I_{1}+I_{2}+I_{3},
$$

where

$$
\begin{aligned}
I_{1} & =\sum_{i=1}^{3} \tilde{f}_{0 i} \partial_{i} D_{t}\left(S_{1}^{2}-S_{1}\right) \dot{\Phi}+\sum_{1 \leq i \neq j \leq 3} \tilde{f}_{i j} \partial_{i j}^{2}\left(S_{1}^{2}-S_{1}\right) \dot{\Phi}+\sum_{i=1}^{3} \tilde{f}_{i i} \partial_{i}^{2}\left(S_{1}^{2}-S_{1}\right) \dot{\Phi} \\
I_{2} & =\sum_{i=1}^{3} \tilde{f}_{0 i}\left[\left(S_{1}^{2}-S_{1}\right), \partial_{i} D_{t}\right] \dot{\Phi}+\sum_{0 \leq i \neq j \leq 3} \tilde{f}_{i j}\left[\left(S_{1}^{2}-S_{1}\right), \partial_{i j}^{2}\right] \dot{\Phi}+\sum_{i=1}^{3} \tilde{f}_{i i}\left[\left(S_{1}^{2}-S_{1}\right), \partial_{i}^{2}\right] \dot{\Phi} \\
I_{3} & =\sum_{1 \leq l \leq 2} C_{l 1}\left\{\sum_{l_{1}+l_{2}=l, l_{1} \geq 1} \tilde{C}_{l_{1} l_{2}}\left(\sum_{i=1}^{3}\left(S_{1}^{l_{1}} \tilde{f}_{0 i}\right) S_{1}^{l_{2}}\left(\partial_{i} D_{t} \dot{\Phi}\right)+\sum_{1 \leq i, j \leq 3}\left(S_{1}^{l_{1}} \tilde{f}_{i j}\right) S_{1}^{l_{2}}\left(\partial_{i j}^{2} \dot{\Phi}\right)\right)\right\}+\left(S_{1}^{2}-S_{1}\right) \tilde{f}_{0} .
\end{aligned}
$$

Similar to (5.14), one has

$$
I_{1} \cdot \mathcal{M}\left(S_{1}^{2}-S_{1}\right) \dot{\Phi}
$$


38

$$
\begin{aligned}
= & D_{t}\left(-\frac{1}{2} a(t) R(t)^{\mu} \sum_{i, j=1}^{3}\left(\tilde{f}_{i j} \partial_{i}\left(S_{1}^{2}-S_{1}\right) \dot{\Phi} \partial_{j}\left(S_{1}^{2}-S_{1}\right) \dot{\Phi}\right)\right) \\
& +\sum_{i=1}^{3} \partial_{i}\left(\frac{1}{2} a(t) R(t)^{\mu} \tilde{f}_{0 i}\left(D_{t}\left(S_{1}^{2}-S_{1}\right) \dot{\Phi}\right)^{2}+a(t) R(t)^{\mu} D_{t}\left(S_{1}^{2}-S_{1}\right) \dot{\Phi} \sum_{j=1}^{3} \tilde{f}_{i j} \partial_{j}\left(S_{1}^{2}-S_{1}\right) \dot{\Phi}\right) \\
& -\frac{1}{2} a(t) R(t)^{\mu}\left(D_{t}\left(S_{1}^{2}-S_{1}\right) \dot{\Phi}\right)^{2} \sum_{i=1}^{3} \partial_{i} f_{0 i}-a(t) L R(t)^{\mu-1} \sum_{i, j=1}^{3}\left(\tilde{f}_{i j} \partial_{i}\left(S_{1}^{2}-S_{1}\right) \dot{\Phi} \partial_{j}\left(S_{1}^{2}-S_{1}\right) \dot{\Phi}\right) \\
& -a(t) R(t)^{\mu} D_{t}\left(S_{1}^{2}-S_{1}\right) \dot{\Phi} \sum_{i, j=1}^{3}\left(\partial_{i} \tilde{f}_{i j} \partial_{j}\left(S_{1}^{2}-S_{1}\right) \dot{\Phi}\right) \\
& +\frac{1}{2} \sum_{i, j=1}^{3}\left(D_{t}\left(a(t) R(t)^{\mu} \tilde{f}_{i j}\right) \partial_{i}\left(S_{1}^{2}-S_{1}\right) \dot{\Phi} \partial_{j}\left(S_{1}^{2}-S_{1}\right) \dot{\Phi}\right) .
\end{aligned}
$$

On the other hand, by the expressions of $\tilde{f}_{i j}, \tilde{f}_{0 i}$ and (5.7), direct observation yields on $B_{T}$

$$
\begin{aligned}
\sum_{i=1}^{3} x_{i} \cdot\left(\frac{1}{2} a(t) R(t)^{\mu} \tilde{f}_{0 i}\left(D_{t}\left(S_{1}^{2}-S_{1}\right) \dot{\Phi}\right)^{2}+a(t) R(t)^{\mu} D_{t}\left(S_{1}^{2}-S_{1}\right) \dot{\Phi} \sum_{j=1}^{3} \tilde{f}_{i j} \partial_{j}\left(S_{1}^{2}-S_{1}\right) \dot{\Phi}\right) \\
=-a(t) R(t)^{\mu}\left(D_{t}\left(S_{1}^{2}-S_{1}\right) \dot{\Phi}\right)^{2} \sum_{i=1}^{3}\left(x_{i} \partial_{i} \dot{\Phi}\right)-a(t) R(t)^{\mu} D_{t}\left(S_{1}^{2}-S_{1}\right) \dot{\Phi} \sum_{i, j=1}^{3}\left(x_{i} \partial_{i} \dot{\Phi} \partial_{j} \dot{\Phi}\right) \\
\quad-(\gamma-1) a(t) R(t)^{\mu} D_{t}\left(S_{1}^{2}-S_{1}\right) \dot{\Phi}\left(D_{t} \dot{\Phi}+\frac{1}{2} \sum_{k=1}^{3}\left(\partial_{k} \dot{\Phi}\right)^{2}\right) \sum_{i=1}^{3} x_{i} \partial_{i}\left(S_{1}^{2}-S_{1}\right) \dot{\Phi} \\
=-(\gamma-1) a(t) R(t)^{\mu+1}\left(D_{t} \dot{\Phi}+\frac{1}{2} \sum_{k=1}^{3}\left(\partial_{k} \dot{\Phi}\right)^{2}\right) \partial_{r}\left(D_{t} S_{1}-D_{t}\right) \dot{\Phi} \cdot\left(\chi\left(\frac{r}{R(t)}\right) \frac{G}{A}+2 \sum_{i=1}^{3} Z_{i}^{2} \dot{\Phi}\right) .
\end{aligned}
$$

Similar to (5.71)-(5.74), we obtain

$$
\begin{aligned}
& \left|\int_{B_{T}} \sum_{i=1}^{3} x_{i} \cdot\left(\frac{1}{2} a(t) R(t)^{\mu} \tilde{f}_{0 i}\left(D_{t}\left(S_{1}^{2}-S_{1}\right) \dot{\Phi}\right)^{2}+a(t) R(t)^{\mu} D_{t}\left(S_{1}^{2}-S_{1}\right) \dot{\Phi} \sum_{j=1}^{3} \tilde{f}_{i j} \partial_{j}\left(S_{1}^{2}-S_{1}\right) \dot{\Phi}\right) d S\right| \\
& \leq C \varepsilon^{2}+C \varepsilon \int_{\Omega_{T}}\left(\sum_{l=0}^{3} R(t)^{\mu-1-\delta+2 l}\left(\nabla_{t, x}^{l} D_{t} \dot{\Phi}\right)^{2}+\sum_{l=0}^{2} R(t)^{\mu-1-3(\gamma-1)+2 l}\left(\nabla_{t, x}^{l} \nabla_{x} \dot{\Phi}\right)^{2}\right) d t d x .
\end{aligned}
$$

Thus, as in (5.44), it follows

$$
\begin{aligned}
& \left|\int_{\Omega_{T}} I_{1} \cdot \mathcal{M}\left(S_{1}^{2}-S_{1}\right) \dot{\Phi} d t d x\right| \\
& \leq C \varepsilon^{2}+C \varepsilon\left(R(T)^{\mu} \int_{S_{T}}\left(D_{t}\left(S_{1}^{2}-S_{1}\right)^{\dot{\Phi}}\right)^{2} d S+R(T)^{\mu-3(\gamma-1)} \int_{S_{T}}\left(\nabla_{x}\left(S_{1}^{2}-S_{1}\right) \dot{\Phi}\right)^{2} d S\right. \\
& \left.\quad+\int_{\Omega_{T}}\left(\sum_{l=0}^{3} R(t)^{\mu-1-\delta+2 l}\left(\nabla_{t, x}^{l} D_{t} \dot{\Phi}\right)^{2}+\sum_{l=0}^{2} R(t)^{\mu-1-3(\gamma-1)+2 l}\left(\nabla_{t, x}^{l} \nabla_{x} \dot{\Phi}\right)^{2}\right) d t d x\right) .
\end{aligned}
$$


Then, similar to (5.45) and (5.51), it follows

$$
\begin{aligned}
& \int_{\Omega_{T}}\left(\left|I_{2}\right|+\left|I_{3}\right|\right) \cdot\left|\mathcal{M}\left(S_{1}^{2}-S_{1}\right) \dot{\Phi}\right| d t d x \\
& \leq C \varepsilon^{2}+C \varepsilon \sum_{l=0}^{2} \int_{\Omega_{T}}\left(R(t)^{\mu-1-\delta+2 l}\left(\nabla_{t, x}^{l} D_{t} \dot{\Phi}\right)^{2}+R(t)^{\mu-1-3(\gamma-1)+2 l}\left(\nabla_{t, x}^{l} \nabla_{x} \dot{\Phi}\right)^{2}\right) d t d x .
\end{aligned}
$$

Substituting (5.74)-(5.76) and (5.80)-(5.81) into (5.70), we complete the proof of Lemma 5.3.

Remark 5.5 Under the assumptions of Theorem 5.1, as the estimation in the proof of Lemma 5.2, we have

$$
\begin{aligned}
& R(T)^{\mu} \int_{S_{T}}\left(D_{t} S^{2} S_{1} \dot{\Phi}\right)^{2} d S+R(T)^{\mu-3(\gamma-1)} \int_{S_{T}}\left(\nabla_{x} S^{2} S_{1} \dot{\Phi}\right)^{2} d S \\
& \quad+\int_{\Omega_{T}}\left(R(t)^{\mu-1-\delta}\left(D_{t} S^{2} S_{1} \dot{\Phi}\right)^{2}+R(t)^{\mu-1-3(\gamma-1)}\left(\nabla_{x} S^{2} S_{1} \dot{\Phi}\right)^{2}\right) d t d x \\
& \leq C \varepsilon^{2}+C \varepsilon \int_{\Omega_{T}} \sum_{l=0}^{2}\left(R(t)^{\mu-1-\delta+2 l}\left(\nabla_{t, x}^{l} D_{t} \dot{\Phi}\right)^{2}+R(t)^{\mu-1-3(\gamma-1)+2 l}\left(\nabla_{t, x}^{l} \nabla_{x} \dot{\Phi}\right)^{2}\right) d t d x \\
& \quad+C \varepsilon \int_{\Omega_{T}}\left(R(t)^{\mu+5-\delta}\left(\nabla_{t, x}^{3} D_{t} \dot{\Phi}\right)^{2}+R(t)^{\mu+5-3(\gamma-1)-\delta}\left(\nabla_{t, x}^{3} \nabla_{x} \dot{\Phi}\right)^{2}\right) d t d x \\
& \quad+C \varepsilon\left(\int_{\Omega_{T}}\left(R(t)^{\mu+5-\delta}\left(\nabla_{t, x}^{3} D_{t} \dot{\Phi}\right)^{2}+R(t)^{\mu+5-3(\gamma-1)-\delta}\left(\nabla_{t, x}^{3} \nabla_{x} \dot{\Phi}\right)^{2}\right) d t d x\right)^{\frac{1}{2}} .
\end{aligned}
$$

Next, we will give the estimates on $\nabla_{t, x}^{4} \dot{\Phi}$ in Lemma 5.4-Lemma 5.6.

Lemma 5.4 (Estimate on $D_{t} S S_{1}^{2} \dot{\Phi}$ and $\nabla_{x} S S_{1}^{2} \dot{\Phi}$ ) Under the assumptions of Theorem 5.1, we have

$$
\begin{aligned}
& R(T)^{\mu} \int_{S_{T}}\left(D_{t} S S_{1}^{2} \dot{\Phi}\right)^{2} d S+R(T)^{\mu-3(\gamma-1)} \int_{S_{T}}\left(\nabla_{x} S S_{1}^{2} \dot{\Phi}\right)^{2} d S \\
& +\int_{\Omega_{T}}\left(R(t)^{\mu-1-\delta}\left(D_{t} S S_{1}^{2} \dot{\Phi}\right)^{2}+R(t)^{\mu-1-3(\gamma-1)}\left(\nabla_{x} S S_{1}^{2} \dot{\Phi}\right)^{2}\right) d t d x \\
\leq & C \varepsilon^{2}+C \varepsilon \sum_{l=0}^{2} \int_{\Omega_{T}}\left(R(t)^{\mu-1-\delta+2 l}\left(\nabla_{t, x}^{l} D_{t} \dot{\Phi}\right)^{2}+R(t)^{\mu-1-3(\gamma-1)+2 l}\left(\nabla_{t, x}^{l} \nabla_{x} \dot{\Phi}\right)^{2}\right) d t d x \\
& +C \varepsilon \int_{\Omega_{T}}\left(R(t)^{\mu+5-\delta}\left(\nabla_{t, x}^{3} D_{t} \dot{\Phi}\right)^{2}+R(t)^{\mu+5-3(\gamma-1)-\delta}\left(\nabla_{t, x}^{3} \nabla_{x} \dot{\Phi}\right)^{2}\right) d t d x \\
& +C \varepsilon\left(\sum_{l=0}^{2} \int_{\Omega_{T}}\left(R(t)^{\mu-1-\delta+2 l}\left(\nabla_{t, x}^{l} D_{t} \dot{\Phi}\right)^{2}+R(t)^{\mu-1-3(\gamma-1)+2 l}\left(\nabla_{t, x}^{l} \nabla_{x} \dot{\Phi}\right)^{2}\right) d t d x\right. \\
& \left.+\int_{\Omega_{T}}\left(R(t)^{\mu+5-\delta}\left(\nabla_{t, x}^{3} D_{t} \dot{\Phi}\right)^{2}+R(t)^{\mu+5-3(\gamma-1)-\delta}\left(\nabla_{t, x}^{3} \nabla_{x} \dot{\Phi}\right)^{2}\right) d t d x\right)^{\frac{1}{2}} .
\end{aligned}
$$

Proof. Applying $S$ to (5.66) yields

$$
S_{1}\left(S S_{1}^{2} \dot{\Phi}-S S_{1} \dot{\Phi}\right)-2 \sum_{i=1}^{3} S Z_{i}^{2} \dot{\Phi}-\chi\left(\frac{r}{R(t)}\right) S\left(\frac{G}{A}\right)=0 \quad \text { on } B_{T} .
$$


40

In addition, direct computation yields

$$
\mathcal{L} Z\left(S_{1}^{2}-S_{1}\right) \dot{\Phi}=Z\left(S_{1}^{2}-S_{1}\right) \mathcal{L} \dot{\Phi}+4 \hat{c}^{2} \Delta Z S_{1} \dot{\Phi}-2 \hat{c}^{2} \Delta Z \dot{\Phi},
$$

and

$$
\begin{aligned}
\mathcal{L} S_{0}\left(S_{1}^{2}-S_{1}\right) \dot{\Phi}= & S_{0}\left(S_{1}^{2}-S_{1}\right) \mathcal{L} \dot{\Phi}-2 L\left(S_{1}^{2}-S_{1}\right) \mathcal{L} \dot{\Phi}+S_{0}\left(4 \hat{c}^{2} \Delta S_{1} \dot{\Phi}-2 \hat{c}^{2} \Delta \dot{\Phi}\right) \\
& -8 L \hat{c}^{2} \Delta S_{1} \dot{\Phi}+4 L \hat{c}^{2} \Delta \dot{\Phi}+3(\gamma-1) \hat{c}^{2} \Delta\left(S_{1}^{2}-S_{1}\right) \dot{\Phi}
\end{aligned}
$$

Similar to (5.69), we obtain

$$
\begin{aligned}
& \int_{\Omega_{T}} \mathcal{L} S\left(S_{1}^{2}-S_{1}\right) \dot{\Phi} \cdot \mathcal{M} S\left(S_{1}^{2}-S_{1}\right) \dot{\Phi} d t d x \\
= & -\int_{B_{T}} \frac{R(t)^{\mu}}{\sqrt{1+L^{2}}} \hat{c}^{2} a(t) D_{t} S\left(S_{1}^{2}-S_{1}\right) \dot{\Phi} \cdot \partial_{r} S\left(S_{1}^{2}-S_{1}\right) \dot{\Phi} d S \\
& +\left(\int_{S_{T}}-\int_{S^{0}}\right) \frac{1}{2} R(t)^{\mu} a(t)\left(D_{t} S\left(S_{1}^{2}-S_{1}\right) \dot{\Phi}+\hat{c}^{2}\left|\nabla_{x} S\left(S_{1}^{2}-S_{1}\right) \dot{\Phi}\right|^{2}\right) d S \\
& +\int_{\Omega_{T}}\left\{\frac{\delta}{2} R(t)^{\mu-1-\delta}\left(D_{t} S\left(S_{1}^{2}-S_{1}\right) \dot{\Phi}\right)^{2}+\frac{\gamma}{2} R(t)^{\mu-1-3(\gamma-1)}(L(5-3 \gamma) a(t)\right. \\
& \left.\left.-R(t) a^{\prime}(t)\right)\left|\nabla_{x} S\left(S_{1}^{2}-S_{1}\right) \dot{\Phi}\right|^{2}\right\} d t d x .
\end{aligned}
$$

As in (5.71), we have

$$
\begin{aligned}
& \int_{B_{T}} \frac{R(t)^{\mu}}{\sqrt{1+L^{2}}} \hat{c}^{2} a(t) D_{t} S\left(S_{1}^{2}-S_{1}\right) \dot{\Phi} \cdot \partial_{r} S\left(S_{1}^{2}-S_{1}\right) \dot{\Phi} d S \\
& =\int_{\Omega_{T}} R(t)^{\mu} \hat{c}^{2} a(t)\left[\Delta\left(D_{t} S S_{1} \dot{\Phi}-D_{t} S \dot{\Phi}\right)\left(\chi\left(\frac{r}{R(t)}\right) S\left(\frac{G}{A}\right)+2 \sum_{i=1}^{3} S Z_{i}^{2} \dot{\Phi}\right)\right] d t d x \\
& \quad+\int_{\Omega_{T}} R(t)^{\mu} \hat{c}^{2} a(t) \sum_{j=1}^{3} \partial_{j}\left(D_{t} S S_{1} \dot{\Phi}-D_{t} S \dot{\Phi}\right)\left(\partial_{j}\left(\chi\left(\frac{r}{R(t)}\right) S \frac{G}{A}\right)+2 \sum_{i=1}^{3} \partial_{j} S Z_{i}^{2} \dot{\Phi}\right) d t d x .
\end{aligned}
$$

Note that, for $C^{1}$-smooth functions $f$ and $g$,

$$
\begin{aligned}
& \int_{\Omega_{T}}\left(x_{i} \partial_{j} f-x_{j} \partial_{i} f\right) g d t d x=\int_{\Omega_{T}}\left(\partial_{j}\left(x_{i} f g\right)-\partial_{i}\left(x_{j} f g\right)\right)-f\left(x_{i} \partial_{j} g-x_{j} \partial_{i} g\right) d t d x \\
= & -\int_{\Omega_{T}} f\left(x_{i} \partial_{j} g-x_{j} \partial_{i} g\right) d t d x .
\end{aligned}
$$

Then

$$
\int_{\Omega_{T}} Z f \cdot g d t d x=-\int_{\Omega_{T}} f \cdot Z g d t d x .
$$

Hence, if we choose $S=Z$, then it follows from (5.88) that

$$
\int_{\Omega_{T}} R(t)^{\mu} \hat{c}^{2} a(t)\left[\Delta\left(D_{t} Z S_{1} \dot{\Phi}-Z D_{t} \dot{\Phi}\right)\left(\chi\left(\frac{r}{R(t)}\right) Z\left(\frac{G}{A}\right)+2 \sum_{i=1}^{3} Z Z_{i}^{2} \dot{\Phi}\right)\right] d t d x
$$




$$
=-\int_{\Omega_{T}} R(t)^{\mu} \hat{c}^{2} a(t)\left[\Delta\left(D_{t} S_{1} \dot{\Phi}-D_{t} \dot{\Phi}\right)\left(\chi\left(\frac{r}{R(t)}\right) Z^{2}\left(\frac{G}{A}\right)+2 \sum_{i=1}^{3} Z^{2} Z_{i}^{2} \dot{\Phi}\right)\right] d t d x
$$

In addition, it follows from (5.2) that

$$
\begin{aligned}
&\left|\chi\left(\frac{r}{R(t)}\right) Z\left(\frac{G}{A}\right)\right| \leq C \varepsilon\left(R(t)^{-\frac{3(\gamma-1)}{2}}\left|S_{1}^{2} \dot{\Phi}\right|+R(t)^{-3(\gamma-1)}\left(\left|Z S_{1}^{2} \dot{\Phi}\right|+\left|Z^{3} \dot{\Phi}\right|\right)+|Z \dot{\Phi}|+\left|Z^{2} \dot{\Phi}\right|\right) \\
&\left|\chi\left(\frac{r}{R(t)}\right) Z^{2}\left(\frac{G}{A}\right)\right| \leq C \varepsilon\left(\left|S_{1}^{2} \dot{\Phi}\right|+\sum_{l=1}^{4}\left|Z^{l} \dot{\Phi}\right|+R(t)^{-\frac{3(\gamma-1)}{2}}\left|Z S_{1}^{2} \dot{\Phi}\right|+R(t)^{-3(\gamma-1)}\left|Z^{2} S_{1}^{2} \dot{\Phi}\right|\right. \\
&+\left.R(t)\left|D_{t} Z^{2} \dot{\Phi}\right|\right)+R(t)^{3(\gamma-1)-1}\left|Z^{2} \dot{\Phi}\right|^{2} .
\end{aligned}
$$

Combining (5.88) and (5.90)-(5.92), we have that from (5.88)

$$
\begin{aligned}
& \quad\left|\int_{B_{T}} \frac{R(t)^{\mu}}{\sqrt{1+L^{2}}} \hat{c}^{2} a(t) D_{t} Z\left(S_{1}^{2}-S_{1}\right) \dot{\Phi} \cdot \partial_{r} Z\left(S_{1}^{2}-S_{1}\right) \dot{\Phi} d S\right| \\
& \leq \quad C \varepsilon \sum_{l=0}^{2} \int_{\Omega_{T}}\left(R(t)^{\mu-1-\delta+2 l}\left(\nabla_{t, x}^{l} D_{t} \dot{\Phi}\right)^{2}+R(t)^{\mu-1-3(\gamma-1)+2 l}\left(\nabla_{t, x}^{l} \nabla_{x} \dot{\Phi}\right)^{2}\right) d t d x \\
& \quad+C \varepsilon \int_{\Omega_{T}}\left(R(t)^{\mu+5-\delta}\left(\nabla_{t, x}^{3} D_{t} \dot{\Phi}\right)^{2}+R(t)^{\mu+5-3(\gamma-1)-\delta}\left(\nabla_{t, x}^{3} \nabla_{x} \dot{\Phi}\right)^{2}\right) d t d x \\
& \quad+\int_{\Omega_{T}} R(t)^{\mu-1}\left|Z^{2} \dot{\Phi}\right|^{2} \cdot\left|\Delta\left(D_{t} S_{1} \dot{\Phi}-D_{t} \dot{\Phi}\right)\right| d t d x .
\end{aligned}
$$

By Hölder inequality and Lemma 2.4, we have

$$
\begin{aligned}
& \left|R(t)^{\mu-1}\left(Z^{2} \dot{\Phi}\right)^{2} \Delta\left(D_{t} S_{1} \dot{\Phi}-D_{t} \dot{\Phi}\right)\right|_{L^{1}\left(\Omega_{T}\right)} \\
& \leq C\left|R(t)^{\mu-1}\left(\nabla_{x} Z \dot{\Phi}\right)^{2} \sum_{l=2}^{3}\left(\left(R(t) \nabla_{x}\right)^{l} D_{t} \dot{\Phi}\right)\right|_{L^{1}\left(\Omega_{T}\right)} \\
& \leq C\left|R(t)^{\frac{2 \mu+5-3 \gamma-\delta}{4}} \nabla_{x} Z \dot{\Phi}\right|_{L^{4}\left(\Omega_{T}\right)}^{2}\left|R(t)^{\frac{\mu-1-\delta}{2}} \sum_{l=2}^{3}\left(\left(R(t) \nabla_{x}\right)^{l} D_{t} \dot{\Phi}\right)\right|_{L^{2}\left(\Omega_{T}\right)} \\
& \leq C \varepsilon \sum_{l=0}^{2}\left(\left|R(t)^{\frac{\mu-1-\delta+2 l}{2}} \nabla_{t, x}^{l} D_{t} \dot{\Phi}\right|_{L^{2}}+\left|R(t)^{\frac{\mu-1-3(\gamma-1)+2 l}{2}} \nabla_{t, x}^{l} \nabla_{x} \dot{\Phi}\right|_{L^{2}}\right)\left|R(t)^{\frac{\mu-1-\delta}{2}} \sum_{l=2}^{3}\left(\left(R(t) \nabla_{x}\right)^{l} D_{t} \dot{\Phi}\right)\right|_{L^{2}} \\
& \quad \text { (Applying Lemma 2.4 (iii) for } \dot{\Phi})
\end{aligned}
$$$$
\leq C \varepsilon\left(\sum_{l=0}^{3}\left|R(t)^{\frac{\mu-1-\delta+2 l}{2}} \nabla_{t, x}^{l} D_{t} \dot{\Phi}\right|_{L^{2}}^{2}+\sum_{l=0}^{2}\left|R(t)^{\frac{\mu-1-3(\gamma-1)+2 l}{2}} \nabla_{t, x}^{l} \nabla_{x} \dot{\Phi}\right|_{L^{2}}^{2}\right),
$$

that gives

$$
\int_{\Omega_{T}} R(t)^{\mu-1}\left|Z^{2} \dot{\Phi}\right|^{2} \cdot\left|\Delta\left(D_{t} S_{1} \dot{\Phi}-D_{t} \dot{\Phi}\right)\right| d t d x
$$


42

$$
\leq C \varepsilon \int_{\Omega_{T}}\left(\sum_{l=0}^{3} R(t)^{\mu-1-\delta+2 l}\left(\nabla_{t, x}^{l} D_{t} \dot{\Phi}\right)^{2}+\sum_{l=0}^{2} R(t)^{\mu-1-3(\gamma-1)+2 l}\left(\nabla_{t, x}^{l} \nabla_{x} \dot{\Phi}\right)^{2}\right) d t d x .
$$

Substituting (5.94) into (5.93) yields

$$
\begin{aligned}
& \left|\int_{B_{T}} \frac{R(t)^{\mu}}{\sqrt{1+L^{2}}} \hat{c}^{2} a(t) D_{t} S\left(S_{1}^{2}-S_{1}\right) \dot{\Phi} \cdot \partial_{r} S\left(S_{1}^{2}-S_{1}\right) \dot{\Phi} d S\right| \\
\leq & C \varepsilon \sum_{l=0}^{2} \int_{\Omega_{T}}\left(R(t)^{\mu-1-\delta+2 l}\left(\nabla_{t, x}^{l} D_{t} \dot{\Phi}\right)^{2}+R(t)^{\mu-1-3(\gamma-1)+2 l}\left(\nabla_{t, x}^{l} \nabla_{x} \dot{\Phi}\right)^{2}\right) d t d x \\
& +C \varepsilon \int_{\Omega_{T}}\left(R(t)^{\mu+5-\delta}\left(\nabla_{t, x}^{3} D_{t} \dot{\Phi}\right)^{2}+R(t)^{\mu+5-3(\gamma-1)-\delta}\left(\nabla_{t, x}^{3} \nabla_{x} \dot{\Phi}\right)^{2}\right) d t d x .
\end{aligned}
$$

Substituting (5.85) and (5.95) into (5.87), it follows

$$
\begin{aligned}
& R(T)^{\mu} \int_{S_{T}}\left(D_{t} Z\left(S_{1}^{2}-S_{1}\right) \dot{\Phi}\right)^{2} d S+R(T)^{\mu-3(\gamma-1)} \int_{S_{T}}\left(\nabla_{x} Z\left(S_{1}^{2}-S_{1}\right) \dot{\Phi}\right)^{2} d S \\
& +\int_{\Omega_{T}}\left(R(t)^{\mu-1-\delta}\left(D_{t} Z\left(S_{1}^{2}-S_{1}\right) \dot{\Phi}\right)^{2}+R(t)^{\mu-1-3(\gamma-1)}\left(\nabla_{x} Z\left(S_{1}^{2}-S_{1}\right) \dot{\Phi}\right)^{2}\right) d t d x \\
\leq & C \varepsilon^{2}+\int_{\Omega_{T}}\left(Z\left(S_{1}^{2}-S_{1}\right) \mathcal{L} \dot{\Phi}+4 \hat{c}^{2} \Delta Z S_{1} \dot{\Phi}-2 \hat{c}^{2} \Delta Z \dot{\Phi}\right) \cdot \mathcal{M} Z\left(S_{1}^{2}-S_{1}\right) \dot{\Phi} d t d x \\
& +C \varepsilon \sum_{l=0}^{2} \int_{\Omega_{T}}\left(R(t)^{\mu-1-\delta+2 l}\left(\nabla_{t, x}^{l} D_{t} \dot{\Phi}\right)^{2}+R(t)^{\mu-1-3(\gamma-1)+2 l}\left(\nabla_{t, x}^{l} \nabla_{x} \dot{\Phi}\right)^{2}\right) d t d x \\
& +C \varepsilon \int_{\Omega_{T}}\left(R(t)^{\mu+5-\delta}\left(\nabla_{t, x}^{3} D_{t} \dot{\Phi}\right)^{2}+R(t)^{\mu+5-3(\gamma-1)-\delta}\left(\nabla_{t, x}^{3} \nabla_{x} \dot{\Phi}\right)^{2}\right) d t d x .
\end{aligned}
$$

Similar to (5.75), we have

$$
\begin{aligned}
& \int_{\Omega_{T}}\left(\left|\hat{c}^{2} \Delta Z S_{1} \dot{\Phi}\right|+\left|\hat{c}^{2} \Delta \dot{\Phi}\right|\right) \cdot\left|\mathcal{M} Z\left(S_{1}^{2}-S_{1}\right) \dot{\Phi}\right| d t d x \\
\leq & C \varepsilon^{2}+C \varepsilon\left(\int_{\Omega_{T}}\left(R(t)^{\mu+5-\delta}\left(\nabla_{t, x}^{3} D_{t} \dot{\Phi}\right)^{2}+R(t)^{\mu+5-3(\gamma-1)-\delta}\left(\nabla_{t, x}^{3} \nabla_{x} \dot{\Phi}\right)^{2}\right) d t d x\right)^{\frac{1}{2}} .
\end{aligned}
$$

As (5.80)-(5.81) and (5.95), we have

$$
\begin{aligned}
& \quad\left|\int_{\Omega_{T}} Z\left(S_{1}^{2}-S_{1}\right) \mathcal{L} \dot{\Phi} \cdot \mathcal{M} Z\left(S_{1}^{2}-S_{1}\right) \dot{\Phi} d t d x\right| \\
& \leq \quad C \varepsilon^{2}+C \varepsilon\left(R(T)^{\mu} \int_{S_{T}}\left(D_{t} Z\left(S_{1}^{2}-S_{1}\right) \dot{\Phi}\right)^{2} d S+R(T)^{\mu-3(\gamma-1)} \int_{S_{T}}\left(\nabla_{x} Z\left(S_{1}^{2}-S_{1}\right) \dot{\Phi}\right)^{2} d S\right. \\
& \left.\quad+\int_{\Omega_{T}}\left(\sum_{l=0}^{2} R(t)^{\mu-1-\delta+2 l}\left(\nabla_{t, x}^{l} D_{t} \dot{\Phi}\right)^{2}+\sum_{l=0}^{2} R(t)^{\mu-1-3(\gamma-1)+2 l}\left(\nabla_{t, x}^{l} \nabla_{x} \dot{\Phi}\right)^{2}\right) d t d x\right) \\
& \quad+C \varepsilon \int_{\Omega_{T}}\left(R(t)^{\mu+5-\delta}\left(\nabla_{t, x}^{3} D_{t} \dot{\Phi}\right)^{2}+R(t)^{\mu+5-3(\gamma-1)-\delta}\left(\nabla_{t, x}^{3} \nabla_{x} \dot{\Phi}\right)^{2}\right) d t d x \\
& \quad+C \varepsilon\left(\int_{\Omega_{T}}\left(R(t)^{\mu+5-\delta}\left(\nabla_{t, x}^{3} D_{t} \dot{\Phi}\right)^{2}+R(t)^{\mu+5-3(\gamma-1)-\delta}\left(\nabla_{t, x}^{3} \nabla_{x} \dot{\Phi}\right)^{2}\right) d t d x\right)^{\frac{1}{2}}
\end{aligned}
$$


Thus, together with (5.96)-(5.98), this yields Lemma 5.4 for the case $S=Z$.

Next, we deal with the case of $S=S_{0}$. From (5.13), we have

$\int_{\Omega_{T}} D_{t} f \cdot g d t d x=\int_{\Omega_{T}} D_{t}(f g)-f D_{t} g d t d x=\left(\int_{S_{T}}-\int_{S^{0}}\right) f g d S-\int_{\Omega_{T}}\left(\frac{3 L}{R(t)} f g+f D_{t} g\right) d t d x$.

If we choose $S=S_{0}$, then it follows from (5.88) and $\left[S_{0}, R(t)^{2} \Delta\right]=0$ that

$$
\begin{aligned}
& \int_{\Omega_{T}} R(t)^{\mu} \hat{c}^{2} a(t)\left[\Delta\left(D_{t} S_{0} S_{1} \dot{\Phi}-D_{t} S_{0} \dot{\Phi}\right)\left(\chi\left(\frac{r}{R(t)}\right) S_{0}\left(\frac{G}{A}\right)+2 \sum_{i=1}^{3} S_{0} Z_{i}^{2} \dot{\Phi}\right)\right] d t d x \\
= & \int_{\Omega_{T}} D_{t}\left(R(t)^{2} \Delta\left(S_{0} S_{1} \dot{\Phi}-S_{0} \dot{\Phi}\right)\right) \cdot R(t)^{\mu-2} \hat{c}^{2} a(t)\left(\chi\left(\frac{r}{R(t)}\right) S_{0}\left(\frac{G}{A}\right)+2 \sum_{i=1}^{3} S_{0} Z_{i}^{2} \dot{\Phi}\right) d t d x \\
= & \left(\int_{S_{T}}-\int_{S^{0}}\right) R(t)^{\mu} \hat{c}^{2} a(t) \Delta\left(S_{0} S_{1} \dot{\Phi}-S_{0} \dot{\Phi}\right)\left(\chi\left(\frac{r}{R(t)}\right) S_{0}\left(\frac{G}{A}\right)+2 \sum_{i=1}^{3} S_{0} Z_{i}^{2} \dot{\Phi}\right) d S \\
& -\int_{\Omega_{T}}\left(R(t)^{2} \Delta\left(S_{0} S_{1} \dot{\Phi}-S_{0} \dot{\Phi}\right)\right) \cdot D_{t}\left(R(t)^{\mu-2} \hat{c}^{2} a(t)\left(\chi\left(\frac{r}{R(t)}\right) S_{0}\left(\frac{G}{A}\right)+2 \sum_{i=1}^{3} S_{0} Z_{i}^{2} \dot{\Phi}\right)\right) d t d x \\
& -\int_{\Omega_{T}} 3 L R(t)^{\mu} \hat{c}^{2} a(t) \Delta\left(S_{0} S_{1} \dot{\Phi}-S_{0} \dot{\Phi}\right)\left(\chi\left(\frac{r}{R(t)}\right) S_{0}\left(\frac{G}{A}\right)+2 \sum_{i=1}^{3} S_{0} Z_{i}^{2} \dot{\Phi}\right) d t d x .
\end{aligned}
$$

And it follows from (5.2) that

$$
\begin{aligned}
& \left|\chi\left(\frac{r}{R(t)}\right) S_{0}\left(\frac{G}{A}\right)\right| \leq C \varepsilon\left(R(t)^{-3(\gamma-1)} \sum_{l=0}^{1}\left(\left|S_{0}^{l} S_{1}^{2} \dot{\Phi}\right|+\left|S_{0}^{l} Z^{2} \dot{\Phi}\right|\right)+\sum_{l=0}^{1}\left(\left|S_{0}^{l} Z \dot{\Phi}\right|\right)\right) \\
& \left|\chi\left(\frac{r}{R(t)}\right) S_{0}^{2}\left(\frac{G}{A}\right)\right| \leq C \varepsilon\left(R(t)^{-3(\gamma-1)} \sum_{l=0}^{2}\left(\left|S_{0}^{l} S_{1}^{2} \dot{\Phi}\right|+\left|S_{0}^{l} Z^{2} \dot{\Phi}\right|\right)+\sum_{l=0}^{2}\left|S_{0}^{l} Z \dot{\Phi}\right|+R(t)\left|S_{0}^{2} D_{t} \dot{\Phi}\right|\right) .
\end{aligned}
$$

Thus, combining (5.88) and (5.100)-(5.102), we have

$$
\begin{aligned}
& \left|\int_{B_{T}} \frac{R(t)^{\mu}}{\sqrt{1+L^{2}}} \hat{c}^{2} a(t) D_{t} S_{0}\left(S_{1}^{2}-S_{1}\right) \dot{\Phi} \cdot \partial_{r} S_{0}\left(S_{1}^{2}-S_{1}\right) \dot{\Phi} d S\right| \\
\leq & C \varepsilon \sum_{l=0}^{2} \int_{\Omega_{T}}\left(R(t)^{\mu-1-\delta+2 l}\left(\nabla_{t, x}^{l} D_{t} \dot{\Phi}\right)^{2}+R(t)^{\mu-1-3(\gamma-1)+2 l}\left(\nabla_{t, x}^{l} \nabla_{x} \dot{\Phi}\right)^{2}\right) d t d x \\
& +C \varepsilon \int_{\Omega_{T}}\left(R(t)^{\mu+5-\delta}\left(\nabla_{t, x}^{3} D_{t} \dot{\Phi}\right)^{2}+R(t)^{\mu+5-3(\gamma-1)-\delta}\left(\nabla_{t, x}^{3} \nabla_{x} \dot{\Phi}\right)^{2}\right) d t d x .
\end{aligned}
$$

Substituting (5.86) and (5.103) into (5.87) yields

$$
\begin{aligned}
& R(T)^{\mu} \int_{S_{T}}\left(D_{t} S_{0}\left(S_{1}^{2}-S_{1}\right) \dot{\Phi}\right)^{2} d S+R(T)^{\mu-3(\gamma-1)} \int_{S_{T}}\left(\nabla_{x} S_{0}\left(S_{1}^{2}-S_{1}\right) \dot{\Phi}\right)^{2} d S \\
& \quad+\int_{\Omega_{T}}\left(R(t)^{\mu-1-\delta}\left(D_{t} S_{0}\left(S_{1}^{2}-S_{1}\right) \dot{\Phi}\right)^{2}+R(t)^{\mu-1-3(\gamma-1)}\left(\nabla_{x} S_{0}\left(S_{1}^{2}-S_{1}\right) \dot{\Phi}\right)^{2}\right) d t d x
\end{aligned}
$$


44

$$
\begin{aligned}
\leq & C \varepsilon^{2}+C \varepsilon \sum_{l=0}^{2} \int_{\Omega_{T}}\left(R(t)^{\mu-1-\delta+2 l}\left(\nabla_{t, x}^{l} D_{t} \dot{\Phi}\right)^{2}+R(t)^{\mu-1-3(\gamma-1)+2 l}\left(\nabla_{t, x}^{l} \nabla_{x} \dot{\Phi}\right)^{2}\right) d t d x \\
& +C \varepsilon \int_{\Omega_{T}}\left(R(t)^{\mu+5-\delta}\left(\nabla_{t, x}^{3} D_{t} \dot{\Phi}\right)^{2}+R(t)^{\mu+5-3(\gamma-1)-\delta}\left(\nabla_{t, x}^{3} \nabla_{x} \dot{\Phi}\right)^{2}\right) d t d x \\
& +\int_{\Omega_{T}}\left(S_{0}\left(S_{1}^{2}-S_{1}\right) \mathcal{L} \dot{\Phi}-2 L\left(S_{1}^{2}-S_{1}\right) \mathcal{L} \dot{\Phi}+S_{0}\left(4 \hat{c}^{2} \Delta S_{1} \dot{\Phi}-2 \hat{c}^{2} \Delta \dot{\Phi}\right)\right. \\
& \left.-8 L \hat{c}^{2} \Delta S_{1} \dot{\Phi}+4 L \hat{c}^{2} \Delta \dot{\Phi}+3(\gamma-1) \hat{c}^{2} \Delta\left(S_{1}^{2}-S_{1}\right) \dot{\Phi}\right) \cdot \mathcal{M} S_{0}\left(S_{1}^{2}-S_{1}\right) \dot{\Phi} d t d x
\end{aligned}
$$

Similar to (5.75), it follows

$$
\begin{aligned}
& \int_{\Omega_{T}}\left(\left|S_{0}\left(\hat{c}^{2} \Delta S_{1} \dot{\Phi}\right)\right|+\left|S_{0}\left(\hat{c}^{2} \Delta \dot{\Phi}\right)\right|+\left|\hat{c}^{2} \Delta S_{1} \dot{\Phi}\right|+\left|\hat{c}^{2} \Delta \dot{\Phi}\right|+\left|\hat{c}^{2} \Delta\left(S_{1}^{2}-S_{1}\right) \dot{\Phi}\right|\right) \cdot\left|\mathcal{M} S_{0}\left(S_{1}^{2}-S_{1}\right) \dot{\Phi}\right| d t d x \\
& \leq C \varepsilon^{2}+C \varepsilon\left(\sum_{l=0}^{2} \int_{\Omega_{T}}\left(R(t)^{\mu-1-\delta+2 l}\left(\nabla_{t, x}^{l} D_{t} \dot{\Phi}\right)^{2}+R(t)^{\mu-1-3(\gamma-1)+2 l}\left(\nabla_{t, x}^{l} \nabla_{x} \dot{\Phi}\right)^{2}\right) d t d x\right. \\
& \left.\quad+\int_{\Omega_{T}}\left(R(t)^{\mu+5-\delta}\left(\nabla_{t, x}^{3} D_{t} \dot{\Phi}\right)^{2}+R(t)^{\mu+5-3(\gamma-1)-\delta}\left(\nabla_{t, x}^{3} \nabla_{x} \dot{\Phi}\right)^{2}\right) d t d x\right)^{\frac{1}{2}}
\end{aligned}
$$

As (5.80)-(5.81) and (5.95), we have

$$
\begin{aligned}
& \quad\left|\int_{\Omega_{T}}\left(\left|S_{0}\left(S_{1}^{2}-S_{1}\right) \mathcal{L} \dot{\Phi}\right|+\left|\left(S_{1}^{2}-S_{1}\right) \mathcal{L} \dot{\Phi}\right|\right) \mathcal{L} \dot{\Phi} \cdot \mathcal{M} S_{0}\left(S_{1}^{2}-S_{1}\right) \dot{\Phi} d t d x\right| \\
& \leq \quad C \varepsilon^{2}+C \varepsilon\left(R(T)^{\mu} \int_{S_{T}}\left(D_{t} Z\left(S_{1}^{2}-S_{1}\right) \dot{\Phi}\right)^{2} d S+R(T)^{\mu-3(\gamma-1)} \int_{S_{T}}\left(\nabla_{x} Z\left(S_{1}^{2}-S_{1}\right) \dot{\Phi}\right)^{2} d S\right. \\
& \left.\quad+\int_{\Omega_{T}}\left(\sum_{l=0}^{2} R(t)^{\mu-1-\delta+2 l}\left(\nabla_{t, x}^{l} D_{t} \dot{\Phi}\right)^{2}+\sum_{l=0}^{2} R(t)^{\mu-1-3(\gamma-1)+2 l}\left(\nabla_{t, x}^{l} \nabla_{x} \dot{\Phi}\right)^{2}\right) d t d x\right) \\
& \quad+C \varepsilon \int_{\Omega_{T}}\left(R(t)^{\mu+5-\delta}\left(\nabla_{t, x}^{3} D_{t} \dot{\Phi}\right)^{2}+R(t)^{\mu+5-3(\gamma-1)-\delta}\left(\nabla_{t, x}^{3} \nabla_{x} \dot{\Phi}\right)^{2}\right) d t d x \\
& \quad+C \varepsilon\left(\int_{\Omega_{T}}\left(R(t)^{\mu+5-\delta}\left(\nabla_{t, x}^{3} D_{t} \dot{\Phi}\right)^{2}+R(t)^{\mu+5-3(\gamma-1)-\delta}\left(\nabla_{t, x}^{3} \nabla_{x} \dot{\Phi}\right)^{2}\right) d t d x\right)^{\frac{1}{2}} .
\end{aligned}
$$

Together with (5.104)-(5.106), Lemma 5.4 is proved for the case of $S=S_{0}$ and it then completes the proof of the lemma.

Lemma 5.5 (The fourth order radial derivative estimates) Under the assumptions of Theorem 5.1, we have

$$
\begin{aligned}
& R(T)^{\mu-\delta} \int_{S_{T}}\left(D_{t} S_{1}^{3} \dot{\Phi}\right)^{2} d S+R(T)^{\mu-3(\gamma-1)-\delta} \int_{S_{T}}\left(\nabla_{x} S_{1}^{3} \dot{\Phi}\right)^{2} d S \\
& +\int_{\Omega_{T}}\left(R(t)^{\mu-1-\delta}\left(D_{t} S_{1}^{3} \dot{\Phi}\right)^{2}+R(t)^{\mu-1-3(\gamma-1)-\delta}\left(\nabla_{x} S_{1}^{3} \dot{\Phi}\right)^{2}\right) d t d x \\
\leq & C \varepsilon^{2}+C \varepsilon \int_{\Omega_{T}} \sum_{l=0}^{2}\left(R(t)^{\mu-1-\delta+2 l}\left(\nabla_{t, x}^{l} D_{t} \dot{\Phi}\right)^{2}+R(t)^{\mu-1-3(\gamma-1)+2 l}\left(\nabla_{t, x}^{l} \nabla_{x} \dot{\Phi}\right)^{2}\right) d t d x
\end{aligned}
$$




$$
\begin{aligned}
& +C \varepsilon \int_{\Omega_{T}}\left(R(t)^{\mu+5-\delta}\left(\nabla_{t, x}^{l} D_{t} \dot{\Phi}\right)^{2}+R(t)^{\mu+5-3(\gamma-1)-\delta}\left(\nabla_{t, x}^{l} \nabla_{x} \dot{\Phi}\right)^{2}\right) d t d x \\
& +C \varepsilon\left(\sum_{l=0}^{2} \int_{\Omega_{T}}\left(R(t)^{\mu-1-\delta+2 l}\left(\nabla_{t, x}^{l} D_{t} \dot{\Phi}\right)^{2}+R(t)^{\mu-1-3(\gamma-1)+2 l}\left(\nabla_{t, x}^{l} \nabla_{x} \dot{\Phi}\right)^{2}\right) d t d x\right. \\
& \left.+\int_{\Omega_{T}}\left(R(t)^{\mu+5-\delta}\left(\nabla_{t, x}^{3} D_{t} \dot{\Phi}\right)^{2}+R(t)^{\mu+5-3(\gamma-1)-\delta}\left(\nabla_{t, x}^{3} \nabla_{x} \dot{\Phi}\right)^{2}\right) d t d x\right)^{\frac{1}{2}} .
\end{aligned}
$$

Remark 5.6 Together with Lemma 5.1-5.5 and Remark 5.3-5.5, we obtain

$$
\begin{aligned}
& \sum_{0 \leq l_{1}+l_{2} \leq 2}\left(R(T)^{\mu} \int_{S_{T}}\left(D_{t} S^{l_{1}} S_{1}^{l_{2}} \dot{\Phi}\right)^{2} d S+R(T)^{\mu-3(\gamma-1)} \int_{S_{T}}\left(\nabla_{x} S^{l_{1}} S_{1}^{l_{2}} \dot{\Phi}\right)^{2} d S\right) \\
+ & \sum_{l_{1}+l_{2}=3, l_{1} \geq 1}\left(R(T)^{\mu} \int_{S_{T}}\left(D_{t} S^{l_{1}} S_{1}^{l_{2}} \dot{\Phi}\right)^{2} d S+R(T)^{\mu-3(\gamma-1)} \int_{S_{T}}\left(\nabla_{x} S^{l_{1}} S_{1}^{l_{2}} \dot{\Phi}\right)^{2} d S\right) \\
+ & R(T)^{\mu-\delta} \int_{S_{T}}\left(D_{t} S_{1}^{3} \dot{\Phi}\right)^{2} d S+R(T)^{\mu-3(\gamma-1)-\delta} \int_{S_{T}}\left(\nabla_{x} S_{1}^{3} \dot{\Phi}\right)^{2} d S \\
+ & \sum_{0 \leq l_{1}+l_{2} \leq 2} \int_{\Omega_{T}}\left(R(t)^{\mu-1-\delta}\left(D_{t} S^{l_{1}} S_{1}^{l_{2}} \dot{\Phi}\right)^{2}+R(t)^{\mu-1-3(\gamma-1)}\left(\nabla_{x} S^{l_{1}} S_{1}^{l_{2}} \dot{\Phi}\right)^{2}\right) d t d x \\
+ & \sum_{l_{1}+l_{2}=3, l_{1} \geq 1} \int_{\Omega_{T}}\left(R(t)^{\mu-1-\delta}\left(D_{t} S^{l_{1}} S_{1}^{l_{2}} \dot{\Phi}\right)^{2}+R(t)^{\mu-1-3(\gamma-1)}\left(\nabla_{x} S^{l_{1}} S_{1}^{l_{2}} \dot{\Phi}\right)^{2}\right) d t d x \\
+ & \int_{\Omega_{T}}\left(R(t)^{\mu-1-\delta}\left(D_{t} S_{1}^{3} \dot{\Phi}\right)^{2}+R(t)^{\mu-1-3(\gamma-1)-\delta}\left(\nabla_{x} S_{1}^{3} \dot{\Phi}\right)^{2}\right) d t d x \\
\leq & C \varepsilon^{2}+C \varepsilon \int_{\Omega_{T}} \sum_{l=0}^{2}\left(R(t)^{\mu-1-\delta+2 l}\left(\nabla_{t, x}^{l} D_{t} \dot{\Phi}\right)^{2}+R(t)^{\mu-1-3(\gamma-1)+2 l}\left(\nabla_{t, x}^{l} \nabla_{x} \dot{\Phi}\right)^{2}\right) d t d x \\
+ & C \varepsilon \int_{\Omega_{T}}\left(R(t)^{\mu+5-\delta}\left(\nabla_{t, x}^{l} D_{t} \dot{\Phi}\right)^{2}+R(t)^{\mu+5-3(\gamma-1)-\delta}\left(\nabla_{t, x}^{l} \nabla_{x} \dot{\Phi}\right)^{2}\right) d t d x \\
+ & C \varepsilon\left(\int _ { \Omega _ { T } } ^ { 2 } \left(R\left(t \sum_{l=0}^{\mu-1-\delta+2 l}\left(\nabla_{t, x}^{l} D_{t} \dot{\Phi}\right)^{2}+R(t)^{\mu-1-3(\gamma-1)+2 l}\left(\nabla_{t, x}^{l} \nabla_{x} \dot{\Phi}\right)^{2}\right) d t d x\right.\right. \\
+ & \left.\int_{\Omega_{T}}\left(R(t)^{\mu+5-\delta}\left(\nabla_{t, x}^{l} D_{t} \dot{\Phi}\right)^{2}+R(t)^{\mu+5-3(\gamma-1)-\delta}\left(\nabla_{t, x}^{l} \nabla_{x} \dot{\Phi}\right)^{2}\right) d t d x\right)^{\frac{1}{2}} .
\end{aligned}
$$

\section{Proof of Lemma 5.5. Set}

$$
\begin{aligned}
N_{1} & =S_{1}^{3}-S_{1}^{2}-2 \sum_{i=1}^{3} Z_{i}^{2} \dot{\Phi} \\
N_{2} & =\chi\left(\frac{r}{R(t)}\right) \frac{1}{A}\left(-\frac{\gamma-1}{R(t)^{2}} \sum_{i=1}^{3}\left(Z_{i} \dot{\Phi}\right)^{2}\left(S_{1}^{2}+\sum_{i=1}^{3} Z_{i}^{2}\right)-\frac{3(\gamma-1) L}{R(t)} \sum_{i=1}^{3} Z_{i} \dot{\Phi} Z_{i}-\frac{2 L}{R(t)} \sum_{i, j=1}^{3} C_{i j} Z_{i} \dot{\Phi} Z_{j}\right. \\
& \left.-\frac{4}{R(t)^{2}} \sum_{i, j=1}^{3} Z_{i} \dot{\Phi} Z_{j} \dot{\Phi} Z_{i} Z_{j}+\frac{1}{R(t)^{2}} \sum_{i, j=1}^{3} C_{i j} Z_{i} \dot{\Phi} Z_{j} \dot{\Phi} S_{1}^{2}-\frac{4}{R(t)^{2}} \sum_{i, j, k=1}^{3} C_{i j k} Z_{i} \dot{\Phi} Z_{j} \dot{\Phi} Z_{k}\right)
\end{aligned}
$$


Then it follows from (5.66) that on $B_{T}$

$$
N \dot{\Phi} \equiv\left(N_{1}-N_{2}\right) \dot{\Phi}=0 .
$$

On the other hand,

$$
\mathcal{L} N \dot{\Phi}=N \mathcal{L} \dot{\Phi}+\left[\mathcal{L}, N_{1}\right] \dot{\Phi}-\left[\mathcal{L}, N_{2}\right] \dot{\Phi}
$$

where

$$
\left[\mathcal{L}, N_{1}\right] \dot{\Phi}=-6 \hat{c}^{2} \Delta S_{1}^{2} \dot{\Phi}+8 \hat{c}^{2} \Delta S_{1} \dot{\Phi}-4 \hat{c}^{2} \Delta \dot{\Phi}
$$

and

$$
\begin{aligned}
\left|\left[\mathcal{L}, N_{2}\right] \dot{\Phi}\right| \leq & C \varepsilon \chi\left(\frac{r}{R(t)}\right)\left(R(t)^{-3(\gamma-1)-2} \sum_{0 \leq l \leq 1}\left(\left|S_{0}^{l} S_{1}^{2} \dot{\Phi}\right|+\left|S_{0}^{l} Z^{2} \dot{\Phi}\right|\right)+R(t)^{-2} \sum_{0 \leq l \leq 2}\left|S_{0}^{l} Z \dot{\Phi}\right|\right. \\
& +R(t)^{-3(\gamma-1)+\delta-2} \sum_{0 \leq l \leq 1} R(t)^{l}\left(\left|\nabla_{x}^{l} S_{1}^{2} \dot{\Phi}\right|+\left|\nabla_{x}^{l} Z^{2} \dot{\Phi}\right|\right)+R(t)^{-1}\left|S_{0}^{2} D_{t} \dot{\Phi}\right| \\
& \left.+R(t)^{-\frac{3(\gamma-1)}{2}+\frac{\delta}{2}-2} \sum_{1 \leq l \leq 2} R(t)^{l}\left|\nabla_{x}^{l} Z \dot{\Phi}\right|+R(t)^{-3(\gamma-1)+1}\left|\nabla_{x}^{2} D_{t} \dot{\Phi}\right|\right)
\end{aligned}
$$

Note that the term $R(t)^{-1} S_{0}^{2} D_{t} \dot{\Phi}$ is on the right hand side of (5.114). Thus, we can not choose the multiplier $\mathcal{M N} \dot{\Phi}$ as in Theorem 4.1 to derive the energy estimate of $N \dot{\Phi}$ from (5.112). Otherwise, we can not control the term $\int_{\Omega_{T}} R(t)^{\mu-1} S_{0}^{2} D_{t} \dot{\Phi} \cdot D_{t} N_{1} \dot{\Phi} d t d x$ in $\int_{\Omega_{T}}\left[\mathcal{L}, N_{2}\right] \dot{\Phi} \cdot \mathcal{M} N \dot{\Phi} d t d x$.

Instead, we will use a new multiplier $\tilde{\mathcal{M}} N \dot{\Phi}=R(t)^{\mu-\delta} D_{t} N \dot{\Phi}$. It follows that

$$
\begin{aligned}
& \int_{\Omega_{T}} \mathcal{L} N \dot{\Phi} \cdot \tilde{\mathcal{M}} N \dot{\Phi} d t d x \\
& =\left(\int_{S_{T}}-\int_{S^{0}}\right) \frac{1}{2} R(t)^{\mu-\delta}\left(\left(D_{t} N \dot{\Phi}\right)^{2}+\hat{c}^{2}\left|\nabla_{x} N \dot{\Phi}\right|^{2}\right) d S \\
& \quad+\int_{\Omega_{T}}\left\{\frac{\delta}{2} R(t)^{\mu-1-\delta}\left(D_{t} N \dot{\Phi}\right)^{2}+\frac{\gamma}{2} R(t)^{\mu-1-3(\gamma-1)-\delta} L(5-3 \gamma)\left|\nabla_{x} N \dot{\Phi}\right|^{2}\right\} d t d x .
\end{aligned}
$$

This yields

$$
\begin{aligned}
& R(T)^{\mu-\delta} \int_{S_{T}}\left(D_{t} N \dot{\Phi}\right)^{2} d S+R(T)^{\mu-3(\gamma-1)-\delta} \int_{S_{T}}\left|\nabla_{x} N \dot{\Phi}\right|^{2} d S \\
& +\int_{\Omega_{T}}\left(R(t)^{\mu-1-\delta}\left(D_{t} N \dot{\Phi}\right)^{2}+R(t)^{\mu-1-3(\gamma-1)-\delta}\left|\nabla_{x} N \dot{\Phi}\right|^{2}\right) d t d x \\
\leq & C \varepsilon^{2}+C \int_{\Omega_{T}}\left(N \mathcal{L} \dot{\Phi}+\left[\mathcal{L}, N_{1}\right] \dot{\Phi}-\left[\mathcal{L}, N_{2}\right] \dot{\Phi}\right) \cdot \tilde{\mathcal{M}} N \dot{\Phi} d t d x .
\end{aligned}
$$

It follows from (5.101) and (5.114) that

$$
\begin{aligned}
& \int_{\Omega_{T}}\left|\left[\mathcal{L}, N_{2}\right] \dot{\Phi}\right| \cdot|\tilde{\mathcal{M}} N \dot{\Phi}| d t d x \\
\leq & C \varepsilon^{2}+C \varepsilon \int_{\Omega_{T}} \sum_{l=0}^{2}\left(R(t)^{\mu-1-\delta+2 l}\left(\nabla_{t, x}^{l} D_{t} \dot{\Phi}\right)^{2}+R(t)^{\mu-1-3(\gamma-1)+2 l}\left(\nabla_{t, x}^{l} \nabla_{x} \dot{\Phi}\right)^{2}\right) d t d x
\end{aligned}
$$




$$
+C \varepsilon \int_{\Omega_{T}}\left(R(t)^{\mu+5-\delta}\left(\nabla_{t, x}^{l} D_{t} \dot{\Phi}\right)^{2}+R(t)^{\mu+5-3(\gamma-1)-\delta}\left(\nabla_{t, x}^{l} \nabla_{x} \dot{\Phi}\right)^{2}\right) d t d x
$$

where we have used

$$
\int_{\Omega_{T}} R(t)^{\mu-3-\delta}\left(\chi\left(\frac{r}{R(t)}\right) Z \dot{\Phi}\right)^{2} d t d x \leq C \varepsilon^{2},
$$

because $|Z \dot{\Phi}| \leq C \varepsilon R(t)^{1-3(\gamma-1)}$ holds for $r>\frac{1}{3} R(t)$.

Thus, by (5.83) and (5.101), we have

$$
\begin{aligned}
& \int_{\Omega_{T}}\left|\left[\mathcal{L}, N_{1}\right] \dot{\Phi}\right| \cdot|\tilde{\mathcal{M}} N \dot{\Phi}| d t d x \\
\leq & \int_{\Omega_{T}}\left|\left[\mathcal{L}, N_{1}\right] \dot{\Phi}\right| \cdot R(t)^{\mu-\delta}\left(\left|D_{t} S_{1}^{3} \dot{\Phi}\right|+\left|D_{t} S_{1}^{2} \dot{\Phi}\right|+\sum_{i=1}^{3}\left|D_{t} Z_{i}^{2} \dot{\Phi}\right|+\left|D_{t} N_{2} \dot{\Phi}\right|\right) d t d x \\
\leq & C \varepsilon^{2}+C \varepsilon \int_{\Omega_{T}} \sum_{l=0}^{2}\left(R(t)^{\mu-1-\delta+2 l}\left(\nabla_{t, x}^{l} D_{t} \dot{\Phi}\right)^{2}+R(t)^{\mu-1-3(\gamma-1)+2 l}\left(\nabla_{t, x}^{l} \nabla_{x} \dot{\Phi}\right)^{2}\right) d t d x \\
& +C \varepsilon \int_{\Omega_{T}}\left(R(t)^{\mu+5-\delta}\left(\nabla_{t, x}^{l} D_{t} \dot{\Phi}\right)^{2}+R(t)^{\mu+5-3(\gamma-1)-\delta}\left(\nabla_{t, x}^{l} \nabla_{x} \dot{\Phi}\right)^{2}\right) d t d x \\
& +C \varepsilon\left(\int_{\Omega_{T}}\left(R(t)^{\mu+5-\delta}\left(\nabla_{t, x}^{l} D_{t} \dot{\Phi}\right)^{2}+R(t)^{\mu+5-3(\gamma-1)-\delta}\left(\nabla_{t, x}^{l} \nabla_{x} \dot{\Phi}\right)^{2}\right) d t d x\right)^{\frac{1}{2}} .
\end{aligned}
$$

Next, for $\int_{\Omega_{T}} N \mathcal{L} \dot{\Phi} \cdot \tilde{\mathcal{M}} N \dot{\Phi} d t d x$, direct computation yields

$$
N \mathcal{L} \dot{\Phi}=M_{1}+M_{2}
$$

where

$$
\begin{aligned}
& M_{1}=\sum_{i=1}^{3} \tilde{f}_{0 i} \partial_{i} D_{t} N \dot{\Phi}+\sum_{i, j=1}^{3} \tilde{f}_{i j} \partial_{i j} N \dot{\Phi} \\
& M_{2}=\sum_{i=1}^{3}\left[\tilde{f}_{0 i} \partial_{i} D_{t}, N\right] \dot{\Phi}+\sum_{i, j=1}^{3}\left[\tilde{f}_{i j} \partial_{i j}, N\right] \dot{\Phi} .
\end{aligned}
$$

Then similar to (5.16), we have

$$
\begin{aligned}
& \left|\int_{\Omega_{T}} M_{1} \cdot \tilde{\mathcal{M}} N \dot{\Phi} d t d x\right| \\
\leq & C \varepsilon^{2}+C \varepsilon\left(R(T)^{\mu-\delta} \int_{S_{T}}\left(D_{t} N \dot{\Phi}\right)^{2} d S+R(T)^{\mu-3(\gamma-1)-\delta} \int_{S_{T}}\left(\nabla_{x} N \dot{\Phi}\right)^{2} d S\right. \\
& +\int_{\Omega_{T}}\left(R(t)^{\mu-1-\delta}\left(D_{t} N \dot{\Phi}\right)^{2}+R(t)^{\mu-1-3(\gamma-1)-\delta}\left(\nabla_{x} N \dot{\Phi}\right)^{2} d t d x\right) .
\end{aligned}
$$

Moreover, by the expression of $N$ and Lemma 5.1-5.4, Remark 5.2-5.3, we have

$$
\left|\int_{\Omega_{T}} M_{1} \cdot \tilde{\mathcal{M}} N \dot{\Phi} d t d x\right|
$$


48

$$
\begin{aligned}
\leq & C \varepsilon^{2}+C \varepsilon \int_{\Omega_{T}} \sum_{l=0}^{2}\left(R(t)^{\mu-1-\delta+2 l}\left(\nabla_{t, x}^{l} D_{t} \dot{\Phi}\right)^{2}+R(t)^{\mu-1-3(\gamma-1)+2 l}\left(\nabla_{t, x}^{l} \nabla_{x} \dot{\Phi}\right)^{2}\right) d t d x \\
& +C \varepsilon \int_{\Omega_{T}}\left(R(t)^{\mu+5-\delta}\left(\nabla_{t, x}^{l} D_{t} \dot{\Phi}\right)^{2}+R(t)^{\mu+5-3(\gamma-1)-\delta}\left(\nabla_{t, x}^{l} \nabla_{x} \dot{\Phi}\right)^{2}\right) d t d x .
\end{aligned}
$$

Then as for (5.18)-(5.19), (5.30), (5.45)-(5.47), (5.51)-(5.52) and (5.57), we obtain

$$
\begin{aligned}
& \left|\int_{\Omega_{T}} M_{2} \cdot \tilde{\mathcal{M}} N \dot{\Phi} d t d x\right| \\
\leq & C \varepsilon^{2}+C \varepsilon \int_{\Omega_{T}} \sum_{l=0}^{2}\left(R(t)^{\mu-1-\delta+2 l}\left(\nabla_{t, x}^{l} D_{t} \dot{\Phi}\right)^{2}+R(t)^{\mu-1-3(\gamma-1)+2 l}\left(\nabla_{t, x}^{l} \nabla_{x} \dot{\Phi}\right)^{2}\right) d t d x \\
& +C \varepsilon \int_{\Omega_{T}}\left(R(t)^{\mu+5-\delta}\left(\nabla_{t, x}^{l} D_{t} \dot{\Phi}\right)^{2}+R(t)^{\mu+5-3(\gamma-1)-\delta}\left(\nabla_{t, x}^{l} \nabla_{x} \dot{\Phi}\right)^{2}\right) d t d x
\end{aligned}
$$

Finally, substituting (5.117), (5.119) and (5.122)-(5.123) into (5.116) completes the proof of the lemma.

As shown in Remark 5.6, the operators $S_{1}$ and $Z$ are used in the energy estimates. However, $R(t) \partial_{i}$ $(1 \leq i \leq 3)$ are equivalent to $S_{1}$ and $Z$ only when $r>\frac{1}{3} R(t)$. Hence, we still need to obtain estimates for $r \leq \frac{1}{3} R(t)$. For this purpose, set $\nu(s)=1-\chi(s)$ supported in $\left[0, \frac{2}{3}\right]$, with $\chi(s)$ defined in (5.67).

Lemma 5.6 (Estimates near $r=0$ ) Under the assumptions of Theorem 5.1, we have

$$
\begin{aligned}
& \int_{S_{T}}\left(R(T)^{\mu} \nu\left(\frac{r}{R(t)}\right)\left(D_{t}\left(R(t) \nabla_{x}\right)^{k} \dot{\Phi}\right)^{2}+R(T)^{\mu-3(\gamma-1)} \nu\left(\frac{r}{R(t)}\right)\left(\nabla_{x}\left(R(t) \nabla_{x}\right)^{k} \dot{\Phi}\right)^{2}\right) d S \\
& +\int_{\Omega_{T}}\left(R(t)^{\mu-1-\delta} \nu\left(\frac{r}{R(t)}\right)\left(D_{t}\left(R(t) \nabla_{x}\right)^{k} \dot{\Phi}\right)^{2}+R(t)^{\mu-1-3(\gamma-1)+2 k} \nu\left(\frac{r}{R(t)}\right)\left(\nabla_{x}\left(R(t) \nabla_{x}\right)^{k} \dot{\Phi}\right)^{2}\right) d t d x \\
& \leq C \varepsilon^{2}+C \varepsilon \int_{\Omega_{T}} \sum_{l=0}^{2}\left(R(t)^{\mu-1-\delta+2 l}\left(\nabla_{t, x}^{l} D_{t} \dot{\Phi}\right)^{2}+R(t)^{\mu-1-3(\gamma-1)+2 l}\left(\nabla_{t, x}^{l} \nabla_{x} \dot{\Phi}\right)^{2}\right) d t d x \\
& +C \varepsilon \int_{\Omega_{T}}\left(R(t)^{\mu+5-\delta}\left(\nabla_{t, x}^{l} D_{t} \dot{\Phi}\right)^{2}+R(t)^{\mu+5-3(\gamma-1)-\delta}\left(\nabla_{t, x}^{l} \nabla_{x} \dot{\Phi}\right)^{2}\right) d t d x
\end{aligned}
$$

and

$$
\begin{aligned}
& \int_{S_{T}}\left(R(T)^{\mu-\delta} \nu\left(\frac{r}{R(t)}\right)\left(D_{t}\left(R(t) \nabla_{x}\right)^{3} \dot{\Phi}\right)^{2}+R(T)^{\mu-\delta-3(\gamma-1)} \nu\left(\frac{r}{R(t)}\right)\left(\nabla_{x}\left(R(t) \nabla_{x}\right)^{3} \dot{\Phi}\right)^{2}\right) d S \\
& \quad+\int_{\Omega_{T}}\left(R(t)^{\mu-1-\delta} \nu\left(\frac{r}{R(t)}\right)\left(D_{t}\left(R(t) \nabla_{x}\right)^{3} \dot{\Phi}\right)^{2}+R(t)^{\mu-\delta-1-3(\gamma-1)} \nu\left(\frac{r}{R(t)}\right)\left(\nabla_{x}\left(R(t) \nabla_{x}\right)^{3} \dot{\Phi}\right)^{2}\right) d t d x \\
& \leq C \varepsilon^{2}+C \varepsilon \int_{\Omega_{T}} \sum_{l=0}^{2}\left(R(t)^{\mu-1-\delta+2 l}\left(\nabla_{t, x}^{l} D_{t} \dot{\Phi}\right)^{2}+R(t)^{\mu-1-3(\gamma-1)+2 l}\left(\nabla_{t, x}^{l} \nabla_{x} \dot{\Phi}\right)^{2}\right) d t d x \\
& \quad+C \varepsilon \int_{\Omega_{T}}\left(R(t)^{\mu+5-\delta}\left(\nabla_{t, x}^{l} D_{t} \dot{\Phi}\right)^{2}+R(t)^{\mu+5-3(\gamma-1)-\delta}\left(\nabla_{t, x}^{l} \nabla_{x} \dot{\Phi}\right)^{2}\right) d t d x
\end{aligned}
$$

Proof. By direct computation, we have

$$
\left(R(t) \partial_{i}\right) \mathcal{L}=\mathcal{L}\left(R(t) \partial_{i}\right) .
$$


This, together with Theorem 4.1, yields for $0 \leq k \leq 2$,

$$
\begin{aligned}
& \int_{S_{T}}\left(R(T)^{\mu} \nu\left(\frac{r}{R(t)}\right)\left(D_{t}\left(R(t) \nabla_{x}\right)^{k} \dot{\Phi}\right)^{2}+R(T)^{\mu-3(\gamma-1)} \nu\left(\frac{r}{R(t)}\right)\left(\nabla_{x}\left(R(t) \nabla_{x}\right)^{k} \dot{\Phi}\right)^{2}\right) d S \\
& +\int_{\Omega_{T}}\left(R(t)^{\mu-1-\delta} \nu\left(\frac{r}{R(t)}\right)\left(D_{t}\left(R(t) \nabla_{x}\right)^{k} \dot{\Phi}\right)^{2}+R(t)^{\mu-1-3(\gamma-1)+2 k} \nu\left(\frac{r}{R(t)}\right)\left(\nabla_{x}\left(R(t) \nabla_{x}\right)^{k} \dot{\Phi}\right)^{2}\right) d t d x \\
& \leq C\left(\int_{\Omega_{T}} R(t)^{\mu-1-3(\gamma-1)}\left|\nu^{\prime}\left(\frac{r}{R(t)}\right)\right|\left(\nabla_{x}(R(t) \nabla)^{k} \dot{\Phi}\right)^{2} d t d x\right)^{\frac{1}{2}} \\
& \quad \times\left(\int_{\Omega_{T}} R(t)^{\mu-1-\delta}\left|\nu^{\prime}\left(\frac{r}{R(t)}\right)\right|\left(D_{t}(R(t) \nabla)^{k} \dot{\Phi}\right)^{2} d t d x\right)^{\frac{1}{2}} \\
& \quad+C \varepsilon^{2}+\int_{\Omega_{T}} \mathcal{L}(R(t) \nabla)^{k} \dot{\Phi} \cdot \nu\left(\frac{r}{R(t)}\right) \mathcal{M}(R(t) \nabla)^{k} \dot{\Phi} d t d x .
\end{aligned}
$$

Note that the function $\nu^{\prime}\left(\frac{r}{R(t)}\right)$ has a compact support away from $r=0$ so that the first term on the right hand side of (5.127) can be estimated as in Remark 5.6.

On the other hand, by a similar argument for $\int_{\Omega_{T}} \mathcal{L} Z^{k} \dot{\Phi} \cdot \mathcal{M} Z^{k} \dot{\Phi} d t d x$ in (5.43), we can obtain

$$
\begin{aligned}
& \left|\int_{\Omega_{T}} \mathcal{L}(R(t) \nabla)^{k} \dot{\Phi} \cdot \nu\left(\frac{r}{R(t)}\right) \mathcal{M}(R(t) \nabla)^{k} \dot{\Phi} d t d x\right| \\
& \leq C \varepsilon^{2}+C \varepsilon \int_{\Omega_{T}} \sum_{l=0}^{2}\left(R(t)^{\mu-1-\delta+2 l}\left(\nabla_{t, x}^{l} D_{t} \dot{\Phi}\right)^{2}+R(t)^{\mu-1-3(\gamma-1)+2 l}\left(\nabla_{t, x}^{l} \nabla_{x} \dot{\Phi}\right)^{2}\right) d t d x \\
& \quad+C \varepsilon \int_{\Omega_{T}}\left(R(t)^{\mu+5-\delta}\left(\nabla_{t, x}^{l} D_{t} \dot{\Phi}\right)^{2}+R(t)^{\mu+5-3(\gamma-1)-\delta}\left(\nabla_{t, x}^{l} \nabla_{x} \dot{\Phi}\right)^{2}\right) d t d x .
\end{aligned}
$$

Then (5.124) follows from (5.127)-(5.128) and Remark 5.6. In addition, similar to (5.124), we obtain (5.125). And this completes the proof of the lemma.

Based on Lemma 5.1-Lemma 5.6 and Remark 5.1-Remark 5.6, we are ready to prove Theorem 5.1.

Proof of Theorem 5.1. By Remark 5.6 and Lemma 5.6, for sufficiently small $\varepsilon>0$, we have

$$
\begin{aligned}
& \sum_{k=0}^{2} \int_{S_{T}}\left(R(T)^{\mu+2 k}\left(\nabla_{t, x}^{k} D_{t} \dot{\Phi}\right)^{2}+R(T)^{\mu-3(\gamma-1)+2 k}\left(\nabla_{t, x}^{k} \nabla_{x} \dot{\Phi}\right)^{2}\right) d S \\
& \quad+\int_{S_{T}}\left(R(T)^{\mu+6-\delta}\left(\nabla_{t, x}^{3} D_{t} \dot{\Phi}\right)^{2}+R(T)^{\mu-3(\gamma-1)+6-\delta}\left(\nabla_{t, x}^{k} \nabla_{x} \dot{\Phi}\right)^{2}\right) d S \\
& \quad+\sum_{k=0}^{2} \int_{\Omega_{T}}\left(R(t)^{\mu-1-\delta+2 k}\left(\nabla_{t, x}^{k} D_{t} \dot{\Phi}\right)^{2}+R(t)^{\mu-1-3(\gamma-1)+2 k}\left(\nabla_{t, x}^{k} \nabla_{x} \dot{\Phi}\right)^{2}\right) d t d x \\
& \quad+\int_{\Omega_{T}}\left(R(t)^{\mu+5-\delta}\left(\nabla_{t, x}^{3} D_{t} \dot{\Phi}\right)^{2}+R(t)^{\mu+5-3(\gamma-1)-\delta}\left(\nabla_{t, x}^{3} \nabla_{x} \dot{\Phi}\right)^{2}\right) d t d x \\
& \leq C \varepsilon^{2}+C \varepsilon\left(\int_{\Omega_{T}} \sum_{l=0}^{2}\left(R(t)^{\mu-1-\delta+2 l}\left(\nabla_{t, x}^{l} D_{t} \dot{\Phi}\right)^{2}+R(t)^{\mu-1-3(\gamma-1)+2 l}\left(\nabla_{t, x}^{l} \nabla_{x} \dot{\Phi}\right)^{2}\right) d t d x\right. \\
& \left.\quad+\int_{\Omega_{T}}\left(R(t)^{\mu+5-\delta}\left(\nabla_{t, x}^{l} D_{t} \dot{\Phi}\right)^{2}+R(t)^{\mu+5-3(\gamma-1)-\delta}\left(\nabla_{t, x}^{l} \nabla_{x} \dot{\Phi}\right)^{2}\right) d t d x\right)^{\frac{1}{2}} .
\end{aligned}
$$




$$
\begin{aligned}
& \int_{\Omega_{T}} \sum_{l=0}^{2}\left(R(t)^{\mu-1-\delta+2 l}\left(\nabla_{t, x}^{l} D_{t} \dot{\Phi}\right)^{2}+R(t)^{\mu-1-3(\gamma-1)+2 l}\left(\nabla_{t, x}^{l} \nabla_{x} \dot{\Phi}\right)^{2}\right) d t d x \\
& \quad+\int_{\Omega_{T}}\left(R(t)^{\mu+5-\delta}\left(\nabla_{t, x}^{l} D_{t} \dot{\Phi}\right)^{2}+R(t)^{\mu+5-3(\gamma-1)-\delta}\left(\nabla_{t, x}^{l} \nabla_{x} \dot{\Phi}\right)^{2}\right) d t d x \\
& \leq C \varepsilon^{2}
\end{aligned}
$$

then (5.2) and (5.3) can be derived directly.

If

$$
\begin{aligned}
& \int_{\Omega_{T}} \sum_{l=0}^{2}\left(R(t)^{\mu-1-\delta+2 l}\left(\nabla_{t, x}^{l} D_{t} \dot{\Phi}\right)^{2}+R(t)^{\mu-1-3(\gamma-1)+2 l}\left(\nabla_{t, x}^{l} \nabla_{x} \dot{\Phi}\right)^{2}\right) d t d x \\
& \quad+\int_{\Omega_{T}}\left(R(t)^{\mu+5-\delta}\left(\nabla_{t, x}^{l} D_{t} \dot{\Phi}\right)^{2}+R(t)^{\mu+5-3(\gamma-1)-\delta}\left(\nabla_{t, x}^{l} \nabla_{x} \dot{\Phi}\right)^{2}\right) d t d x \\
& \geq C \varepsilon^{2}
\end{aligned}
$$

then it follows from (5.129) that

$$
\begin{aligned}
& \int_{\Omega_{T}} \sum_{l=0}^{2}\left(R(t)^{\mu-1-\delta+2 l}\left(\nabla_{t, x}^{l} D_{t} \dot{\Phi}\right)^{2}+R(t)^{\mu-1-3(\gamma-1)+2 l}\left(\nabla_{t, x}^{l} \nabla_{x} \dot{\Phi}\right)^{2}\right) d t d x \\
& +\int_{\Omega_{T}}\left(R(t)^{\mu+5-\delta}\left(\nabla_{t, x}^{l} D_{t} \dot{\Phi}\right)^{2}+R(t)^{\mu+5-3(\gamma-1)-\delta}\left(\nabla_{t, x}^{l} \nabla_{x} \dot{\Phi}\right)^{2}\right) d t d x \\
\leq & C \varepsilon\left(\int_{\Omega_{T}} \sum_{l=0}^{2}\left(R(t)^{\mu-1-\delta+2 l}\left(\nabla_{t, x}^{l} D_{t} \dot{\Phi}\right)^{2}+R(t)^{\mu-1-3(\gamma-1)+2 l}\left(\nabla_{t, x}^{l} \nabla_{x} \dot{\Phi}\right)^{2}\right) d t d x\right. \\
& \left.+\int_{\Omega_{T}}\left(R(t)^{\mu+5-\delta}\left(\nabla_{t, x}^{l} D_{t} \dot{\Phi}\right)^{2}+R(t)^{\mu+5-3(\gamma-1)-\delta}\left(\nabla_{t, x}^{l} \nabla_{x} \dot{\Phi}\right)^{2}\right) d t d x\right)^{\frac{1}{2}},
\end{aligned}
$$

which implies

$$
\begin{aligned}
& \int_{\Omega_{T}} \sum_{l=0}^{2}\left(R(t)^{\mu-1-\delta+2 l}\left(\nabla_{t, x}^{l} D_{t} \dot{\Phi}\right)^{2}+R(t)^{\mu-1-3(\gamma-1)+2 l}\left(\nabla_{t, x}^{l} \nabla_{x} \dot{\Phi}\right)^{2}\right) d t d x \\
& \quad+\int_{\Omega_{T}}\left(R(t)^{\mu+5-\delta}\left(\nabla_{t, x}^{l} D_{t} \dot{\Phi}\right)^{2}+R(t)^{\mu+5-3(\gamma-1)-\delta}\left(\nabla_{t, x}^{l} \nabla_{x} \dot{\Phi}\right)^{2}\right) d t d x \\
& \leq C \varepsilon^{2}
\end{aligned}
$$

Substituting this into (5.129) derives (5.2) and (5.3), and then it completes the proof of Theorem 5.1.

\section{Proofs of Theorem 1.1 and 1.2.}

To complete the proof of Theorem 1.2, as in [14], the following estimate is needed. 
Lemma 6.1. For $1 \leq t \leq T_{0}$ and $k_{0} \geq 4$, we have that for a smooth function $\varphi(t, x) \in H^{k_{0}}\left(\Omega_{T}\right)$

$$
\sum_{0 \leq l \leq k_{0}-4}\left|t^{l} \nabla^{l+1} \varphi(t, x)\right|^{2} \leq C_{0} t^{-3} \int_{S_{T}} \sum_{0 \leq l \leq k_{0}-1}\left|t^{l} \nabla^{l+1} \varphi(t, x)\right|^{2} d x .
$$

Proof. For any $t_{1} \in\left[1, T_{0}\right]$, set

$$
\left(t^{\prime}, x^{\prime}\right)=\frac{1}{t_{1}}(t, x)
$$

Then

$$
\nabla_{t, x}^{k} \varphi=\frac{1}{t_{1}^{k}} \nabla_{t^{\prime}, x^{\prime}}^{k} \varphi, \quad \forall k \in \mathbb{N} .
$$

Define $D_{*}=\left\{\left(t^{\prime}, x^{\prime}\right): t^{\prime}=1,\left|x^{\prime}\right| \leq L\right\}$. Then by the Sobolev imbedding theorem and by noting that $D_{*}$ satisfies the uniform interior cone condition,

$$
\left.\left|\nabla_{t^{\prime}, x^{\prime}} \varphi\right|^{2}\left(1, x^{\prime}\right)\left|\leq C \int_{D_{*}} \sum_{0 \leq l \leq 3}\right| \nabla_{t^{\prime}, x^{\prime}}^{l+1} \varphi\right|^{2}\left(1, x^{\prime}\right) d x^{\prime} .
$$

In view of (6.2), one has

$$
\begin{aligned}
\left|\nabla_{t, x} \varphi\right|^{2}\left(t_{1}, x\right) & =\frac{1}{t_{1}^{2}}\left|\nabla_{t^{\prime}, x^{\prime}} \varphi\right|^{2}\left(1, x^{\prime}\right) \\
& \leq \frac{C}{t_{1}^{2}} \int_{D_{*}} \sum_{0 \leq l \leq 3}\left|\nabla_{t^{\prime}, x^{\prime}}^{l+1} \varphi\right|^{2}\left(1, x^{\prime}\right) d x^{\prime} \\
& =\frac{C}{t_{1}^{2}} \int_{S_{T}} \sum_{0 \leq l \leq 3}\left|t_{1}^{l+1} \nabla_{t, x}^{l+1} \varphi\right|^{2}\left(t_{1}, x\right) \frac{1}{t_{1}^{3}} d x \\
& =\frac{C}{t_{1}^{3}} \int_{S_{T}} \sum_{0 \leq l \leq 3}\left|t_{1}^{l} \nabla_{t, x}^{l+1} \varphi\right|^{2}\left(t_{1}, x\right) d x .
\end{aligned}
$$

This yields (6.1) for $l=0$. The cases of $1 \leq l \leq k_{0}-4$ can be estimated similarly and this completes the proof of the lemma.

We now first prove Theorem 1.2 as follows.

Proof of Theorem 1.2. It follows from Lemma 6.1 that, for $0 \leq t \leq T$,

$$
\left\{\begin{array}{l}
\sum_{0 \leq l \leq 1}\left|R(t)^{l} \nabla_{x}^{l+1} \dot{\Phi}\right|^{2} \leq C R(t)^{-3} \int_{S_{t}} \sum_{0 \leq l \leq 3}\left|R(t)^{l} \nabla_{x}^{l+1} \dot{\Phi}\right|^{2} d S \\
\left|R(t) D_{t}^{2} \dot{\Phi}\right|^{2} \leq C R(t)^{-3} \int_{S_{t}} \sum_{0 \leq l \leq 3}\left|R(t)^{1+l} \nabla_{x}^{l} D_{t}^{2} \dot{\Phi}\right|^{2} d S \\
\sum_{0 \leq l \leq 1}\left|R(t)^{l} \nabla_{x}^{l} D_{t} \dot{\Phi}\right|^{2} \leq C R(t)^{-3} \int_{S_{t}} \sum_{0 \leq l \leq 3}\left|R(t)^{l} \nabla_{x}^{l} D_{t} \dot{\Phi}\right|^{2} d S .
\end{array}\right.
$$

On the other hand, (5.2) gives

$$
\left\{\begin{array}{l}
\int_{S_{t}} \sum_{0 \leq l \leq 2}\left|R(t)^{l} \nabla_{x}^{l+1} \dot{\Phi}\right|^{2} d S \leq C \varepsilon^{2} R(t)^{-\mu+3(\gamma-1)}, \\
\int_{S_{t}} \sum_{0 \leq l \leq 2}\left|R(t)^{l} \nabla_{x}^{l} D_{t} \dot{\Phi}\right|^{2} d S \leq C \varepsilon^{2} R(t)^{-\mu}
\end{array}\right.
$$


and

$$
\left\{\begin{array}{l}
\int_{S_{t}} \sum_{0 \leq l \leq 2}\left|R(t)^{1+l} \nabla_{x}^{l+2} \dot{\Phi}\right|^{2} d S \leq C \varepsilon^{2} R(t)^{-\mu+3(\gamma-1)+\delta} \\
\int_{S_{t}} \sum_{0 \leq l \leq 2}\left|R(t)^{1+l} \nabla_{x}^{1+l} D_{t} \dot{\Phi}\right|^{2} d S \leq C \varepsilon^{2} R(t)^{-\mu+\delta} \\
\int_{S_{t}} \sum_{0 \leq l \leq 3}\left|R(t)^{1+l} \nabla_{x}^{l} D_{t}^{2} \dot{\Phi}\right|^{2} d S \leq C \varepsilon^{2} R(t)^{-\mu+\delta}
\end{array}\right.
$$

Hence, we obtain

$$
\left\{\begin{array}{l}
\left|\nabla_{x} \dot{\Phi}\right| \leq C \varepsilon R(t)^{-3(\gamma-1)+\frac{\delta}{2}}, \quad\left|R(t)^{l-1} D_{t}^{l} \dot{\Phi}\right| \leq C \varepsilon R(t)^{-3(\gamma-1)},(l=1,2) \\
\left|R(t) \nabla_{x} D_{t} \dot{\Phi}\right| \leq C \varepsilon R(t)^{-3(\gamma-1)+\frac{\delta}{2}}, \quad\left|R(t) \nabla_{x}^{2} \dot{\Phi}\right| \leq C \varepsilon R(t)^{-\frac{3(\gamma-1)-\delta}{2}}
\end{array}\right.
$$

On the other hand, by the stream line equation

$$
\frac{d x_{i}(t)}{d t}=\frac{L x_{i}}{R(t)}, \quad x_{i}(0)=x_{i}^{0}, \quad(i=1,2,3)
$$

we have

$$
x_{i}(t)=x_{i}^{0} R(t) \quad(i=1,2,3),
$$

where $\left(x_{1}^{0}, x_{2}^{0}, x_{3}^{0}\right)$ is the initial point. Then integrating along the stream line, we have for $1<\gamma<\frac{4}{3}$ that

$$
\left|R(t) \nabla_{x} \dot{\Phi}(t, x(t))\right| \leq\left|\nabla_{x} \dot{\Phi}(0, x(0))\right|+\int_{0}^{t}\left|D_{t}\left(R(t) \nabla_{x} \dot{\Phi}\right)\right| d t \leq C \varepsilon\left(1+R(t)^{1-3(\gamma-1)+\frac{\delta}{2}}\right),
$$

which implies

$$
\left|\nabla_{x} \dot{\Phi}\right| \leq C \varepsilon\left(R(t)^{-1}+R(t)^{-3(\gamma-1)+\frac{\delta}{2}}\right) \leq C \varepsilon R(t)^{-3(\gamma-1)+\frac{\delta}{2}} .
$$

Thus, the first inequality in (5.1) is proved.

On the other hand, it follows from Remark 5.6 and Theorem 5.1 that

$$
\begin{aligned}
& \quad \sum_{0 \leq l_{1}+l_{2} \leq 2}\left(R(t)^{\mu} \int_{S_{t}}\left(D_{t} S^{l_{1}} S_{1}^{l_{2}} \dot{\Phi}\right)^{2} d S+R(t)^{\mu-3(\gamma-1)} \int_{S_{t}}\left(\nabla_{x} S^{l_{1}} S_{1}^{l_{2}} \dot{\Phi}\right)^{2} d S\right) \\
& \quad+\sum_{l_{1}+l_{2}=3, l_{1} \geq 1}\left(R(t)^{\mu} \int_{S_{t}}\left(D_{t} S^{l_{1}} S_{1}^{l_{2}} \dot{\Phi}\right)^{2} d S+R(t)^{\mu-3(\gamma-1)} \int_{S_{t}}\left(\nabla_{x} S^{l_{1}} S_{1}^{l_{2}} \dot{\Phi}\right)^{2} d S\right) \\
& \leq C \varepsilon^{2} .
\end{aligned}
$$

Noticing that for $r>\frac{1}{3} R(t), r \sim R(t)$ holds. Then (6.10) implies

$$
\begin{aligned}
& \sum_{0 \leq l \leq 2}\left(R(t)^{\mu+2 l} \int_{S_{t} \cap\left\{r>\frac{1}{3} R(t)\right\}}\left(\nabla_{x}^{l} Z D_{t} \dot{\Phi}\right)^{2} d S+R(t)^{\mu-3(\gamma-1)+2 l} \int_{S_{t} \cap\left\{r>\frac{1}{3} R(t)\right\}}\left(\nabla_{x}^{l+1} Z \dot{\Phi}\right)^{2} d S\right) \\
& \leq C \varepsilon^{2} .
\end{aligned}
$$


Together with Lemma 6.1, this yields for $r>\frac{1}{3} R(t)$,

$$
\begin{aligned}
& \left|Z D_{t} \dot{\Phi}\right|^{2} \leq C R(t)^{-3} \int_{S_{t} \cap\left\{r>\frac{1}{3} R(t)\right\}} \sum_{0 \leq l \leq 2}\left|R(t)^{l} \nabla_{x}^{l} Z D_{t} \dot{\Phi}\right|^{2} d S \leq C \varepsilon R(t)^{-\mu-3}, \\
& \left|\nabla_{x} D_{t} \dot{\Phi}\right|^{2} \leq C R(t)^{-3} \int_{S_{t} \bigcap\left\{r>\frac{1}{3} R(t)\right\}} \sum_{0 \leq l \leq 2}\left|R(t)^{l} \nabla_{x}^{l+1} Z \dot{\Phi}\right|^{2} d S \leq C \varepsilon R(t)^{-\mu+3(\gamma-1)-3} .
\end{aligned}
$$

Subsequently, one has

$$
\left|Z D_{t} \dot{\Phi}\right| \leq C \varepsilon R(t)^{-3(\gamma-1)}, \quad\left|\nabla_{x} D_{t} \dot{\Phi}\right| \leq C \varepsilon R(t)^{-\frac{3(\gamma-1)}{2}} .
$$

In addition, for $1<\gamma<\frac{4}{3}$ and $r>\frac{1}{3} R(t)$, integrating along the stream yields

$$
|Z \dot{\Phi}(t, x(t))| \leq|Z \dot{\Phi}(0, x(0))|+\int_{0}^{t}\left|D_{t}(Z \dot{\Phi})\right| d t \leq C \varepsilon\left(1+R(t)^{1-3(\gamma-1)}\right) \leq C \varepsilon R(t)^{1-3(\gamma-1)} .
$$

Note that the generic constant $C$ appeared in this section depends only on the initial data. Then we can choose the constant $M=2 C$ in (5.1)-(5.2) for small $\varepsilon>0$ so that (5.1) and (5.2) hold. In this case, by the Bernoulli law (1.7), we have $c^{2}(\rho)=c^{2}(\hat{\rho})-(\gamma-1) D_{t} \dot{\Phi}-\frac{\gamma-1}{2}\left|\nabla_{x} \dot{\Phi}\right|^{2}$, which gives $C R(t)^{3(1-\gamma)}-C \varepsilon R(t)^{3(1-\gamma)}<c^{2}(\rho)<C R(t)^{3(1-\gamma)}+C \varepsilon R(t)^{3(1-\gamma)}$. Thus, one obtains $c^{2}(\rho) \sim$ $R(t)^{3(1-\gamma)}>0$ for any $t \geq 0$ and small $\varepsilon>0$. Therefore, the proof of Theorem 1.2 is completed by the local existence result in Theorem 3.1 and continuation argument.

Finally, Theorem 1.1 follows.

Proof of Theorem 1.1. Under the assumptions of Theorem 1.1, it follows from Theorem 3.1 and the smallness of $L$ that (1.1)-(1.2) has a local solution $(\rho(t, x), u(t, x))$ that satisfies

$$
\left\|\rho_{0}(1, x)-1\right\|_{H^{4}\left(S^{1}\right)}+\|u(1, x)\|_{H^{4}\left(S^{1}\right)}<\delta_{0},
$$

and

$$
\text { rot } u(1, x) \equiv 0,
$$

where $S^{1}=\{x:|x|=1+L\}, \delta_{0}>0$ is a small number depending only on $L$ and $\varepsilon_{0}$. This, together with Theorem 1.2 for the time $t \geq 1$, yields Theorem 1.1.

Acknowledgement. The authors wish to express their gratitude to Professor Yang Tong, the City University of Hong Kong, for his interests in this problem and many very fruitful discussions. In particular, Professor Yang Tong gave many suggestions and comments that led to substantial improvement of the presentation.

\section{References}

[1] S. Alinhac, Blowup of small data solutions for a class of quasilinear wave equations in two space dimensions, Ann. of Math. 149 (1): 97-127 (1999)

[2] S. Alinhac, Blowup of small data solutions for a class of quasilinear wave equations in two space dimensions. II, Acta Math. 182, no. 1, 1-23 (1999)

[3] J. Y. Chemin, Dynamique des gaz à masse totale finie, Asymptotic Anal. 3, no. 3, 215-220 (1990) 
[4] D. Christodoulou, Global solutions of nonlinear hyperbolic equations for small initial data, Comm. Pure Appl. Math. 39, no. 2, 267-282 (1986)

[5] D. Christodoulou, Shuang Miao, Compressible flow and Euler's equations, arXiv:1212.2867 (2012)

[6] R. Courant, K. O. Friedrichs, Supersonic flow and shock waves, Interscience Publishers Inc., New York, 1948.

[7] D. Coutand, S. Shkoller, Well-posedness in smooth function spaces for moving-boundary 1-D compressible Euler equations in physical vacuum, Comm. Pure Appl. Math. 64, no. 3, 328-366 (2011)

[8] D. Coutand, S. Shkoller, Well-posedness in smooth function spaces for the moving-boundary threedimensional compressible Euler equations in physical vacuum, Arch. Ration. Mech. Anal. 206, no. 2, 515-616 (2012)

[9] M. D’Abbicco, S. Lucente, M. Reissig, Semi-linear wave equations with effective damping, Chin. Ann. Math. Ser. B 34, no. 3, 345-380 (2013)

[10] M. Grassin, Global smooth solutions to Euler equations for a perfect gas, Indiana Univ. Math. J. 47, no. 4, 1397-1432 (1998)

[11] J. Jang, N. Masmoudi, Well-posedness for compressible Euler equations with physical vacuum singularity, Comm. Pure Appl. Math. 62, no. 10, 1327-1385 (2009)

[12] J. Jang, N. Masmoudi, Well-posedness of compressible Euler equations in a physical vacuum, Comm. Pure Appl. Math. 68, no. 1, 61-111 (2015)

[13] S. Klainerman, The null condition and global existence to nonlinear wave equations, Nonlinear systems of partial differential equations in applied mathematics, Part 1 (Santa Fe, N.M., 1984), 293-326, Lectures in Appl. Math., 23, Amer. Math. Soc., Providence, RI, 1986.

[14] Jun Li, Ingo Witt, Huicheng Yin, Global multidimensional shock waves for 2-D and 3-D unsteady potential flow equations, arXiv:1310.3470 (2013)

[15] Changshou Lin, Interpolation inequalities with weights, Comm. Partial Differential Equations 11, no. $14,1515-1538$ (1986)

[16] Tai-Ping Liu, Zhouping Xin, Tong Yang, Vacuum states for compressible flow, Discrete Contin. Dynam. Systems 4, no. 1, 1-32 (1998)

[17] A. Majda, Compressible fluid flow and systems of conservation laws in several space variables, Applied Mathematical Sciences, 53. Springer-Verlag, New York, 1984.

[18] T. Makino, S. Ukai, S. Kawashima, Sur la solution à support compact de l'équations d'Euler compressible, Japan J. Appl. Math. 3, no. 2, 249-257 (1986)

[19] D. Serre, Solutions classiques globales des équations d'Euler pour un fluide parfait compressible, Ann. Inst. Fourier (Grenoble) 47, no. 1, 139-153 (1997)

[20] J. Speck, Shock formation in small-data solutions to $3 D$ quasilinear wave equations, arXiv: 1407.6320 (2014) 
[21] J. Wirth, Wave equations with time-dependent dissipation. I. Non-effective dissipation, J. Differential Equations 222, no. 2, 487-514 (2006)

[22] J. Wirth, Wave equations with time-dependent dissipation. II. Effective dissipation, J. Differential Equations 232, no. 1, 74-103 (2007)

[23] Zhouping Xin, Blowup of smooth solutions to the compressible Navier-Stokes equation with compact density, Comm. Pure Appl. Math. 51, no. 3, 229-240 (1998)

[24] Gang Xu, Huicheng Yin, On global multidimensional supersonic flows with vacuum states at infinity, Arch. Ration. Mech. Anal., Vol.218, No.3, 1189-1238 (2015)

[25] Huicheng Yin, Lin Zhang, The global existence and large time behavior of smooth compressible fluids in infinitely expanding balls, II: 3D Navier-Stokes equations, Preprint (2016)

[26] Huicheng Yin, Wenbin Zhao, The global existence and large time behavior of smooth compressible fluids in infinitely expanding balls, III: 3D Boltzmann equation, Preprint (2016)

[27] Huicheng Yin, Formation and construction of a shock wave for 3-D compressible Euler equations with the spherical initial data, Nagoya Math. J. 175, 125-164 (2004) 